\title{
Solutions, in Particular Dilute Solutions of Nonelectrolytes: A Review
}

\author{
Emmerich Wilhelm ${ }^{1}$ iD
}

Received: 1 February 2021 / Accepted: 16 August 2021 / Published online: 15 November 2021

(c) The Author(s) 2021

\begin{abstract}
The liquid state is one of the three principal states of matter and arguably the most important one; and liquid mixtures represent a large research field of profound theoretical and practical interest. This topic is of importance in many areas of the applied sciences, such as in chemical engineering, geochemistry, the environmental sciences, biophysics and biomedical technology. First, I will concisely present a review of important concepts from classical thermodynamics of nonelectrolyte solutions; this will be followed by a survey of (semi-)empirical approaches to representing the composition and temperature dependence of selected thermodynamic mixture properties, and finally the focus will be on dilute binary nonelectrolyte solutions where one component, a supercritical solute, is present in much smaller quantity than the other component, called the solvent. Partial molar properties in the limit of infinite dilution (indicated by a superscript $\infty$ ) are of particular interest. For instance, activity coefficients (Lewis-Randall (LR) convention) are customarily used to characterize mixing behavior, and infinite-dilution values $\gamma_{i}^{\mathrm{LR}, \infty}$ provide a convenient route for obtaining binary parameters for several popular solution models. When discussing solute $(j)$-solvent $(i)$ interactions in solutions where the solute is supercritical, the Henry fugacity $h_{j, i}(T, P)$, also known as Henry's law (HL) constant, is a measurable thermodynamic key quantity. Its temperature dependence yields information on the partial molar enthalpy change on solution $\Delta H_{j}^{\infty}(T, P)$, while its pressure dependence yields information on the partial molar volume $V_{j}^{\mathrm{L},{ }_{\infty}}(T, P)$ of solute $j$ in the liquid phase (superscript L). I will clarify issues frequently overlooked, touch upon solubility data reduction and correlation, report a few recent high-precision experimental results on dilute aqueous solutions of supercritical nonelectrolytes, and show the equivalency of results for caloric quantities (e.g. $\Delta H_{j}^{\infty}$ ) obtained via van 't Hoff analysis of high-precision solubility data with directly measured calorimetric data.
\end{abstract}

Keywords Mixtures/solutions - Residual properties $\cdot$ Excess molar properties $\cdot$ LewisRandall (LR) rule/Henry's law (HL) · Activity coefficients · Generalized Kohler equation · Solubility of gases in liquids/van 't Hoff analysis

Communicated in part by Emmerich Wilhelm as an Invited Lecture at the 47th IUPAC World Chemistry Congress "IUPAC Centenary Celebration" within the Special Symposium Homage to Eduard Hála (1919-1989), Paris, France, 7-12 July 2019.

Extended author information available on the last page of the article 
Any sufficiently advanced technology is indistinguishable from magic.

Arthur C. Clarke, Profiles of the Future: An Inquiry into the Limits of the Possible, revised edition. Harper \& Row, New York, 1973.

How often have we walked this narrow thoroughfare without having noticed that little shop? Or, if noticed, passed it by? Let us just try the door ... it opens ... a bell tinkles ... What an odd shop ... What can it have for sale? Shall we go in? Avram Davidson, editor, Magic for Sale, Ace Science Fiction, 1983.

\section{Introduction}

\subsection{Preliminaries}

Physical chemistry and chemical physics (if they are, indeed, fields apart) of liquids and liquid mixtures or solutions are indispensable parts of many areas of the pure and applied sciences, such as chemistry, physics, biophysics, chemical engineering, and geoscience, among others. In particular, this large and fundamentally important research field forms bridges between topics where work on macromolecules, colloids, glasses, liquid crystals and biological materials constitutes the highly active and rapidly expanding field of softmatter chemical physics [1-19]. Most processes of interest in chemical engineering are carried out in fluid solution phases, and nonelectrolyte solutions constitute, perhaps, the most important subfield which will be considered in this review. Based on thermodynamics, molecular physics and statistical mechanics, the three main reasons for the enormous effort invested into experimental, theoretical and computer-based research in this field are:

(I) It is hoped that by studying mixture/solution properties and solubilities we will steadily improve our knowledge on interactions between molecules in bulk liquid phases. Any advance in this area will directly benefit science in general, and separation industry in particular, since the most common processes there are predominantly based on vapor-liquid equilibria (VLE) involving multicomponent systems. A classic book in this area, combining thermodynamic theory with experimental practice, has been authored by Hála et al. [20].

(II) The appearance of new physical phenomena not found with the pure liquid components is scientifically fascinating as well as challenging. It adds a new dimension to thermo-physical research and opens the door for novel ideas and developments.

(III) Science-based chemical engineering is one of the most important segments of a modern society, with fluid phase separation technology and chemical/biochemical product design being cornerstones of the field. Solvents have always been the mainstay of the chemical industry, and associated technological problems must be resolved as fast as possible; that includes restrictions aimed at preserving the quality of human life in general, i.e., environmental (sustainability, toxicology, etc.) $[21,22]$ and specific health issues. The practitioners involved must deal with them efficiently and pragmatically.

Experiments are the fundament of science: they provide the basis for inductive reasoning (known informally as bottom-up reasoning), which after amplifying, logically ordering and generalizing our experimental observations leads to hypotheses and then theories, and thus to new knowledge. In contradistinction, deduction (informally known as top-down reasoning), orders and explicates already existing knowledge, thereby leading to predictions 
which may be corroborated experimentally, or falsified [23]. In fact, a theory has no value in science unless it is possible to test it experimentally. Though correct in principle, from a practical point of view the following problem exists: the huge number of potentially useful solubility data connected with binary, ternary, quaternary, etc., systems at different temperatures $T$ and pressures $P$ effectively precludes the experimental determination of mixture/solution properties and solubilities for all but a few representative key systems of physical-chemical/technological interest. This is best illustrated by calculating the number of multi-component solutions containing $c$ components, which can be formed out of, say, $p=500$ chemicals (this is a reasonable estimate of the number of solvents generally available to chemists working in academic, governmental and industrial research laboratories). The number of multi-component solutions is given by the $c$-combination

$$
C_{c}^{p}=\left(\begin{array}{l}
p \\
c
\end{array}\right)=\frac{p !}{c !(p-c) !}=\frac{p(p-1) \cdots(p-(c-1))}{c !} .
$$

Hence, $\quad C_{2}^{500}=0.12475 \times 10^{6}$ different binary solutions may be formed, $C_{3}^{500}=20.7085 \times 10^{6}$ different ternary solutions, $C_{4}^{500}=2.573031125 \times 10^{9}$ different quaternary solutions, and so forth. Thus, reliable and effective prediction methods for thermodynamic properties of fluids, pure and mixed, are an indispensable tool of the trade.

In order to facilitate development and discussion of ideas, I consider it helpful to introduce here a few physical concepts that will be discussed in more detail later on (see Sect. 2.3). The obvious power and utility of an equation-of-state (EOS) has stimulated research in this field for about 150 years, that is, since van der Waals (vdW). He was one of the first to utilize molecular theory to describe semi-quantitatively thermodynamic properties of liquid mixtures in terms of pure-component properties [24-28]. It was based on his then newly developed celebrated volumetric equation of state for pure fluids,

$$
\left(P+\frac{a}{V^{2}}\right)(V-b)=R T,
$$

or alternatively written,

$$
P=\frac{R T}{V-b}-\frac{a}{V^{2}}
$$

Here, $V$ is the molar volume, and $R$ denotes the molar gas constant. The parameter $b$ is known as the covolume and allows for the finite hard size of the molecules, and the averaged attractive intermolecular interaction in the real fluid leads to a correction of the pressure amounting to $a / V^{2}$.

The vdW equation of state is cubic in $V$, that is,

$$
V^{3}-\left(b+\frac{R T}{P}\right) V^{2}+\frac{a}{P} V-\frac{a b}{P}=0,
$$

and such a cubic expression (this is a minimum requirement) is capable of representing both liquid-like and vapor-like molar volumes at sufficiently low temperatures (via the Maxwell equal-area rule). It yields also a critical point (indicated by a subscript c) with critical temperature $T_{\mathrm{c}}$, critical pressure $P_{\mathrm{c}}$ and critical molar volume $V_{\mathrm{c}}$. For the first time, properties of both liquid and vapor could be described by an equation of state using molecular-based parameters, which feature is prerequisite for using an EOS in the calculation of vapor-liquid equilibria. A frequently used alternative form of the vdW equation of state is 


$$
Z \equiv \frac{P V}{R T}=\frac{1}{1-b / V}-\frac{a}{R T V}
$$

where $Z$ is known as the compression factor. As best seen with Eq. 5, in the limit as $V \rightarrow \infty$ the vdW equation yields the perfect-gas (pg) [29], or ideal-gas, equation of state, that is,

$$
Z^{\mathrm{pg}}=\frac{P V^{\mathrm{pg}}}{R T}=1
$$

All pressure-explicit equations of state should satisfy this limit. The term "equation of state" most frequently refers to the equilibrium relation between pressure, volume, temperature and composition of the uniform fluid phase, in the absence of special external force fields. ${ }^{1}$ Most conveniently, the composition is characterized by the mole fractions $x_{i}$ of the components, i.e.,

$$
x_{i} \equiv n_{i} / \sum_{i}^{c} n_{i}=n_{i} / n, \sum_{i}^{c} x_{i}=1 .
$$

Here, $n_{i}$ is the amount of substance of component $i$ in the mixture, $n \equiv \sum_{i}^{c} n_{i}$ is the total amount of substance, and for a pure substance $x_{i}=1$.

Based on his equation of state, Eq. 2, van der Waals formulated his corresponding states theorem (CST) for pure fluids. However, the derived universal critical compression factor

$$
Z_{\mathrm{c}, \mathrm{vdW}}=\frac{P_{\mathrm{c}, \mathrm{vdW}} V_{\mathrm{c}, \mathrm{vdW}}}{R T_{\mathrm{c}, \mathrm{vdW}}}=\frac{3}{8},
$$

is considerably larger than common experimental values. Although first derived for a vdW fluid, it was shown later that CST is much more generally applicable: it is, in fact, valid for any EOS with two adjustable parameters (two-parameter CST), and, as shown by Pitzer [36], it can be derived from statistical mechanics.

\footnotetext{
1 The thermodynamic systems treated in this review are generally referred to as simple systems. By definition, such systems are macroscopically homogeneous, isotropic, uncharged, non-reactive, and large enough to neglect surface effects. In addition, simple systems are not acted upon, for instance, by electrostatic or magnetic fields; in addition, the fluid samples are small enough so that the influence of the earth's gravitational field is not detected in a variation of properties with the height of the containing vessel. Pressure is the only mechanical force considered, causing contraction or dilation of the fluid. Near the critical point, many thermodynamic properties (and transport properties as well) show anomalies linked to the divergence of the fluid's isothermal compressibility [29-31].

$$
\beta_{T}=-V^{-1}(\partial V / \partial P)_{T}=\rho^{-1}(\partial \rho / \partial P)_{T}
$$

where $\rho$ denotes the mass density. Thus, associated with the large compressibility in a near-critical fluid (say, within $1 \mathrm{~K}$ from the critical point), the presence of the gravitational field will cause the local value of the density to vary with height, i.e., macroscopic density gradients (gravitational sedimentation) develop due to compression under the fluid's own weight. Over the height of a few centimeters, they can cause density variations as large as $10 \%$ [32-34]. Note that the divergence of the compressibility gives rise to divergencies of the isobaric expansion coefficient and the isobaric heat capacity, etc., and measurements close to the critical point become significantly distorted. For experiments on fluid systems under terrestrial laboratory conditions and sufficiently removed from the critical region the influence of the earth's gravitational field is generally ignored. However, for interpreting precision measurements in the critical region and to test theoretical predictions of critical phenomena, experiments taking advantage of the microgravity environment of space (space laboratories) are indispensable and have indeed been performed [35].
} 
Nevertheless, from such a volumetric vdW-type EOS, for any given composition, $P V T$ information may be obtained. Using classical thermodynamics, isobaric residual properties and isochoric (isometric) residual properties can be computed, and vapor-liquid phase equilibria (VLE) can be calculated (for definitions see Sect. 2). However, for many practical applications concerning liquid solutions, the focus is on the direct modeling of the molar excess Gibbs energy $G^{\mathrm{E}}\left(T, P,\left\{x_{i}\right\}\right)$ and related quantities, such as activity coefficients (see Sect. 2), though in principle an adequate PVTx equation of state implies a reliable $G^{\mathrm{E}}$. Note that only PVTx systems comprised of chemically non-reactive components will be considered. With few exceptions, the following general system of notation will be used throughout:

- Solutions/mixtures are of prime interest, and their molar single-phase properties, such as the molar volume $V$, will be represented by the plain symbol $M$; additional superscripts (such as E, identifying an excess property, or $\mathrm{R}$, indicating a residual property) will be attached as needed. Thus, I have adopted the nomenclature suggested by Van Ness and Abbott [17].

- A total property of a single-phase multicomponent solution is either indicated by a superscript $\mathrm{t}$, or represented by the product $n M$, i.e., $M^{\mathrm{t}} \equiv n M ; n=\sum_{i} n_{i}$ denotes the total amount (of substance) in the phase, and $n_{i}$ is the amount (of substance) of component $i$. Again, the suggestion of Van Ness and Abbott [17] is adopted.

- Molar single-phase pure-substance properties will be characterized by a superscript asterisk and, if needed, identified by a subscript, i.e., $M_{i}^{*}, i=1,2, \ldots$;

- Partial molar properties referring to a component $i$ in solution will be identified by a subscript, i.e., $M_{i}, i=1,2, \ldots$; additional superscripts/subscripts will be attached as needed. Since a superscript asterisk characterizes a pure-substance property, no overbar is needed to indicate a partial molar property.

The notation adopted in this review facilitates reading equations and avoids as much as possible index-cluttering.

\subsection{Thermodynamic Fundamentals}

Consider a single-phase, multicomponent PVTx system, either open or closed, where the amounts $n_{i}, n_{j}, n_{k}, \ldots$ of components $i, j, k, \ldots$ may vary either because of interchange of matter with the surroundings, or because of chemical reactions within the system, or both. The fundamental equation for a change of state of a phase, also known as the fundamental property relation, or the differential form of the fundamental equation in the internal energy representation, reads

$$
\mathrm{d}(n U)=T \mathrm{~d}(n S)-P \mathrm{~d}(n V)+\sum_{i}^{c}\left[\frac{\partial(n U)}{\partial n_{i}}\right]_{n S, n V, n_{j \neq i}} \mathrm{~d} n_{i},
$$

and, equivalently,

$$
\mathrm{d}(n S)=\frac{1}{T} \mathrm{~d}(n U)+\frac{P}{T} \mathrm{~d}(n V)+\sum_{i}^{c}\left[\frac{\partial(n S)}{\partial n_{i}}\right]_{n U, n V, n_{j \neq i}} \mathrm{~d} n_{i},
$$


in the entropy representation $[17,37]$. Here, $n U=U^{\mathrm{t}}\left(n S, n V, n_{1}, n_{2}, \ldots\right)$ denotes the extensive total internal energy (indicated by the superscript $\mathrm{t}$ ), and $U$ the intensive molar internal energy, $n S=S^{\mathrm{t}}\left(n U, n V, n_{1}, n_{2}, \ldots\right)$ denotes the extensive total entropy, and $S$ the intensive molar entropy.

The intensive parameter furnished by the first-order partial derivative of the total internal energy with respect to the amount of substance $n_{i}$ of component $i$,

$$
\left[\frac{\partial(n U)}{\partial n_{i}}\right]_{n S, n V, n_{j \neq i}} \equiv \mu_{i},
$$

is called the chemical potential of component $i$ in the mixture. It is an intensive conceptual state function. And from Eq. 10 one obtains

$$
\left[\frac{\partial(n S)}{\partial n_{i}}\right]_{n U, n V, n_{j \neq i}}=-\frac{\mu_{i}}{T} .
$$

Hence the fundamental property relation Eq. 9 can be written in a more compact form,

$$
\mathrm{d}(n U)=T \mathrm{~d}(n S)-P \mathrm{~d}(n V)+\sum_{i}^{c} \mu_{i} \mathrm{~d} n_{i},
$$

while the fundamental property relation Eq. 10 becomes

$$
\mathrm{d}(n S)=\frac{1}{T} \mathrm{~d}(n U)+\frac{P}{T} \mathrm{~d}(n V)-\sum_{i}^{c} \frac{\mu_{i}}{T} \mathrm{~d} n_{i} .
$$

Equations 13 and 14 are fundamental because they completely specify all changes that can take place in simple, single-phase, multicomponent PVTx systems, either open or closed, and they form the basis of extremum principles predicting equilibrium states. However, I reiterate that in this review only simple, non-reacting fluid systems will be covered.

The fundamental property relations for open single-phase systems (either in the internal energy representation, Eq. 13, or in the entropy representation, Eq. 14), that is, the differential forms of the fundamental equations for open systems, can be integrated over the change in the amount of substance at constant values of the intensive quantities $\left\{T,-P, \mu_{i}\right\}$ or $\left\{1 / T, P / T,-\mu_{i} / T\right\}$, respectively, and immediately yield the fundamental equations for an open, single-phase, multicomponent PVTx system:

$$
n U=T(n S)-P(n V)+\sum_{i}^{c} \mu_{i} n_{i},
$$

in the internal energy representation, and

$$
n S=\frac{1}{T}(n U)+\frac{P}{T}(n V)-\sum_{i}^{c} \frac{\mu_{i}}{T} n_{i},
$$

in the entropy representation. These equations are also known as the integrated forms of the fundamental equations for a change of the state of a phase; and the state functions $n U\left(n S, n V,\left\{n_{i}\right\}\right)$ and $n S\left(n U, n V,\left\{n_{i}\right\}\right)$ are commonly known as primary functions, or as cardinal functions, or as thermodynamic potentials. 
Alternatively, Eqs. 15 and 16 can be regarded as a consequence of Euler's theorem on homogeneous functions, which asserts the following: if $f\left(z_{1}, z_{2}, \ldots\right)$ is a homogeneous function of degree $k$ in the variables $\left\{z_{1}, z_{2}, \ldots\right\}$, that is, if it satisfies for any value of the constant scaling parameter $\lambda$ the relation

$$
f\left(\lambda z_{1}, \lambda z_{2}, \ldots\right)=\lambda^{k} f\left(z_{1}, z_{2}, \ldots\right),
$$

it must also satisfy

$$
k f\left(z_{1}, z_{2}, \ldots\right)=\sum_{i} z_{i}\left(\partial f / \partial z_{i}\right)_{z_{j \neq i}}, i=1,2, \ldots
$$

In thermodynamics only homogeneous functions of degree $k=0$ and $k=1$ are important. The former are known as intensive functions, and the latter are known as extensive functions. Based on the homogeneous first-order properties of both $U^{\mathrm{t}}$ and $S^{\mathrm{t}}$, that is,

$$
\begin{gathered}
U^{\mathrm{t}}\left(\lambda S^{\mathrm{t}}, \lambda V^{\mathrm{t}}, \lambda n_{1}, \lambda n_{2}, \ldots\right)=\lambda U^{\mathrm{t}}\left(S^{\mathrm{t}}, V^{\mathrm{t}}, n_{1}, n_{2}, \ldots\right), \\
S^{\mathrm{t}}\left(\lambda U^{\mathrm{t}}, \lambda V^{\mathrm{t}}, \lambda n_{1}, \lambda n_{2}, \cdots\right)=\lambda S^{\mathrm{t}}\left(U^{\mathrm{t}}, V^{\mathrm{t}}, n_{1}, n_{2}, \cdots\right),
\end{gathered}
$$

where for a more compact notation, in analogy to $U^{\mathrm{t}}$ and $S^{\mathrm{t}}, n V$ has been replaced by $V^{\mathrm{t}}$, use of Eq. 18 with $k=1$, in conjunction with Eqs. 13 and 14, yields Eqs. 15 and 16, respectively. The corresponding sets of extensive variables, i.e., $\left\{n S, n V, n_{1}, n_{2}, \ldots\right\}$ for the internal energy representation, and $\left\{n U, n V, n_{1}, n_{2}, \ldots\right\}$ for the entropy representation, are called independent canonical or natural variables. With a satisfactory fundamental equation established, all thermodynamic equilibrium properties of a PVTx phase can be calculated by fairly simple mathematical manipulations, that is, by combinations of appropriate derivatives of the corresponding primary function (thermodynamic potential); it is for this reason that they are called fundamental equations.

As indicated by Eq. 13, the parameters $T,-P$ and $\mu_{i}$ are partial derivatives of $n U=U^{\mathrm{t}}\left(n S, n V, n_{1}, n_{2}, \cdots\right)$ and are thus also functions of $\left\{n S, n V, n_{1}, n_{2}, \cdots\right\}$ :

$$
\begin{gathered}
{[\partial(n U) / \partial(n S)]_{n V,\left\{n_{i}\right\}}=T, \text { hence } T=T\left(n S, n V, n_{1}, n_{2}, \ldots\right),} \\
{[\partial(n U) / \partial(n V)]_{n S,\left\{n_{i}\right\}}=-P, \text { hence } P=P\left(n S, n V, n_{1}, n_{2}, \ldots\right),} \\
{\left[\partial(n U) / \partial n_{i}\right]_{n S, n V,\left\{n_{j \neq i}\right\}}=\mu_{i}, \text { hence } \mu_{i}=\mu_{i}\left(n S, n V, n_{1}, n_{2}, \ldots\right) .}
\end{gathered}
$$

$T, P$ and $\mu_{i}$ are each homogeneous zeroth-order functions (intensive functions) in the independent extensive variables $n S, n V$ and $\left\{n_{i}\right\}$, and any relation expressing an intensive parameter in terms of independent extensive parameters, as indicated by Eqs. 21-23, is called a general equation of state. A single equation of state does not contain complete information on the thermodynamic properties of the system. However, the complete set of these three equations of state is equivalent to the fundamental equation and contains all thermodynamic information. Analogous comments apply to the fundamental property relation in the entropy representation, Eq. 14, leading to the corresponding general equations of state as indicated below: 


$$
\begin{gathered}
{[\partial(n S) / \partial(n U)]_{n V,\left\{n_{i}\right\}}=\frac{1}{T}, \text { hence } \frac{1}{T}=\frac{1}{T}\left(n U, n V, n_{1}, n_{2}, \ldots\right),} \\
{[\partial(n S) / \partial(n V)]_{n U,\left\{n_{i}\right\}}=\frac{P}{T}, \text { hence } \frac{P}{T}=\frac{P}{T}\left(n U, n V, n_{1}, n_{2}, \ldots\right),} \\
{\left[\partial(n S) / \partial n_{i}\right]_{n U, n V,\left\{n_{j \neq i}\right\}}=-\frac{\mu_{i}}{T}, \text { hence } \frac{\mu_{i}}{T}=\frac{\mu_{i}}{T}\left(n U, n V, n_{1}, n_{2}, \ldots\right) .}
\end{gathered}
$$

For constant-composition fluids (and thus also for pure fluids), $T=T\left(n U, n V, n_{1}, n_{2}, \cdots\right)$, or explicitly resolved for the extensive total internal energy $n U=U^{\mathrm{t}}$,

$$
U^{\mathrm{t}}=U^{\mathrm{t}}\left(T, n V, n_{1}, n_{2}, \ldots\right) .
$$

This type of equation is known as the caloric equation of state.

Clearly, by using Eqs. 25 and 27 we obtain either a pressure-explicit equation of state

$$
P=P\left(T, n V, n_{1}, n_{2}, \ldots\right),
$$

or, when resolved for the extensive total volume $n V=V^{\mathrm{t}}$, a volume-explicit equation of state

$$
V^{\mathrm{t}}=V^{\mathrm{t}}\left(T, P, n_{1}, n_{2}, \ldots\right) .
$$

A well-known example of a volume-explicit EOS is the virial equation in pressure, and a well-known example of a pressure-explicit EOS is the van der Waals equation. Note, however, that most equations of state in practical use are explicit in pressure.

In the fundamental property relations for an open single-phase PVTx system in both the internal energy representation and the entropy representation, the extensive properties are the mathematically independent variables, while the intensive parameters are derived. Clearly, this situation does not conform to experimental practice. The choice of $n S$ and $n V$ as independent extensive variables in Eq. 13, and of $n U$ and $n V$ as independent extensive variables in Eq. 14, is not convenient: experiment-based experience shows that the conjugate intensive parameters $\{T, P\}$ and $\{1 / T, P / T\}$, respectively, are much more easily measured and controlled. In order to describe the system behavior when one or more of its intensive parameters are held constant (for instance, in isothermal or isobaric processes), alternative versions of the fundamental equations are necessary in which one or more of the extensive parameters are replaced by the conjugate intensive parameter(s) without loss of information. The appropriate formal mathematical technique for generating equivalent alternative thermodynamic potentials is the Legendre transformation [37-40].

Equation 15 suggests the definition of $u s e f u l$ alternative energy-based primary functions related to $n U$ and with total differentials (fundamental property relations) consistent with Eq. 13, but with a set of canonical variables different from $\left\{n S, n V,\left\{n_{i}\right\}\right\}$, while Eq. 16 suggests the definition of useful alternative entropy-based primary functions related to $n S$ and with total differentials (fundamental property relations) consistent with Eq. 14, but with a set of canonical variables different from $\left\{n U, n V,\left\{n_{i}\right\}\right\}$.

Consider the exact (total) differential

$$
\mathrm{d} f^{(0)}=c_{1} \mathrm{~d} X_{1}+c_{2} \mathrm{~d} X_{2}+\cdots+c_{n} \mathrm{~d} X_{n},
$$

pertaining to the function $f^{(0)}$ with $n$ independent variables $X_{i}$, 


$$
f^{(0)}=f^{(0)}\left(X_{1}, X_{2}, \cdots, X_{n}\right),
$$

where

$$
c_{i}=\left(\partial f^{(0)} / \partial X_{i}\right)_{X_{j \neq i}}
$$

Consider now the function obtained by subtracting the product of $X_{1}$ with its conjugate partial derivative $c_{1}$ from the base function $f^{(0)}$, Eq. 31:

$$
f^{(1)}=f^{(0)}-c_{1} X_{1} \text {. }
$$

The corresponding total differential reads

$$
\mathrm{d} f^{(1)}=\mathrm{d} f^{(0)}-c_{1} \mathrm{~d} X_{1}-X_{1} \mathrm{~d} c_{1},
$$

and with Eq. 30 one obtains

$$
\mathrm{d} f^{(1)}=-X_{1} \mathrm{~d} c_{1}+c_{2} \mathrm{~d} X_{2}+c_{3} \mathrm{~d} X_{3}+\cdots+c_{n} \mathrm{~d} X_{n} .
$$

Comparison of Eq. 30 with Eq. 35 shows that the original variable $X_{1}$ and its conjugate $c_{1}$ have interchanged their roles (and a minus sign was introduced): for such an interchange it suffices to subtract $c_{1} X_{1}=\left(\partial f^{(0)} / \partial X_{1}\right)_{X_{j \neq 1}} X_{1}$ from the base function to yield the firstorder partial Legendre transform,

$$
f^{(1)}=f^{(1)}\left(c_{1}, X_{2}, X_{3}, \ldots, X_{n}\right)=f^{(0)}\left[c_{1}\right],
$$

which is frequently identified by a bracket notation indicated above. This Legendre transform represents a new function with the independent variables $\left\{c_{1}, X_{2}, X_{3}, \ldots, X_{n}\right\}$ being the canonical (or natural) variables.

The second-order partial Legendre transform is obtained via

$$
f^{(2)}=f^{(0)}-c_{1} X_{1}-c_{2} X_{2},
$$

which yields the total differential

$$
\begin{aligned}
\mathrm{d} f^{(2)} & =\mathrm{d} f^{(0)}-c_{1} \mathrm{~d} X_{1}-X_{1} \mathrm{~d} c_{1}-c_{2} \mathrm{~d} X_{2}-X_{2} \mathrm{~d} c_{2} \\
& =-X_{1} \mathrm{~d} c_{1}-X_{2} \mathrm{~d} c_{2}+c_{3} \mathrm{~d} X_{3}+\cdots+c_{n} \mathrm{~d} X_{n} .
\end{aligned}
$$

Hence

$$
f^{(2)}=f^{(2)}\left(c_{1}, c_{2}, X_{3}, \ldots, X_{n}\right)=f^{(0)}\left[c_{1}, c_{2}\right],
$$

Analogously, the Legendre transformation of higher order $p$ of the base function $f^{(0)}$ that introduces the partial derivatives $\left\{c_{1}, c_{2}, \ldots, c_{p}\right\}$ into $f^{(0)}$ reads

$$
\begin{aligned}
f^{(p)} & =f^{(0)}-\sum_{i=1}^{p} c_{i} X_{i}=f^{(p)}\left(c_{1}, c_{2}, \ldots, c_{p}, X_{p+1}, X_{p+2}, \ldots, X_{n}\right) \\
& \equiv f^{(0)}\left[c_{1}, c_{2}, \ldots, c_{p}\right],
\end{aligned}
$$

and the associated total differential is 


$$
\mathrm{d} f^{(p)}=\mathrm{d} f^{(0)}\left[c_{1}, c_{2}, \ldots, c_{p}\right]=\sum_{i=1}^{p}\left(-X_{i}\right) \mathrm{d} c_{i}+\sum_{k=p+1}^{n} c_{k} \mathrm{~d} X_{k} .
$$

The complete Legendre transform, i.e., the transform of order $p=n$, replaces all variables by the conjugate partial derivatives, and vanishes identically for any system; this follows directly from the definition:

$$
f^{(n)}=f^{(0)}-\sum_{i=1}^{n} c_{i} X_{i}=f^{(n)}\left(c_{1}, c_{2}, \ldots, c_{n}\right) \equiv f^{(0)}\left[c_{1}, c_{2}, \ldots, c_{n}\right]=0 .
$$

The associated differential expression reads

$$
0=\mathrm{d} f^{(0)}\left[c_{1}, c_{2}, \ldots, c_{n}\right]=\sum_{i=1}^{n}\left(-X_{i}\right) \mathrm{d} c_{i} .
$$

In thermodynamic theory, the complete Legendre transform of the internal energy $n U$ of an open PVTx phase with $c$ components has all extensive canonical variables replaced by their conjugate intensive variables, thus yielding the null function

$$
0=n U\left[T,-P, \sum_{i}^{c} \mu_{i}\right]=n U-T(n S)+P(n V)-\sum_{i}^{c} \mu_{i} n_{i},
$$

and correspondingly

$$
0=-(n S) \mathrm{d} T+(n V) \mathrm{d} P-\sum_{i}^{c} n_{i} \mathrm{~d} \mu_{i},
$$

with canonical variables $\left\{T, P,\left\{\mu_{i}\right\}\right\}$. This property of the complete Legendre transform gives rise to the Gibbs-Duhem equation, which represents an important relation between the intensive parameters $T, P$ and $\left\{\mu_{i}\right\}$ of the system and shows that they are not independent of each other.

When focusing on the fundamental equation in the entropy representation for an open, single-phase, multicomponent (c components) PVTx system, Eq. 16, the complete Legendre transform of the total entropy $n S$ (again all extensive canonical variables are replaced by their conjugate intensive variables) is identically zero, thus yielding the null function

$$
0=n S\left[\frac{1}{T}, \frac{P}{T},-\sum_{i}^{c} \frac{\mu_{i}}{T}\right]=n S-\frac{1}{T}(n U)-\frac{P}{T}(n V)+\sum_{i}^{c} \frac{\mu_{i}}{T} n_{i},
$$

and correspondingly,

$$
0=-(n U) \mathrm{d}\left(\frac{1}{T}\right)-(n V) \mathrm{d}\left(\frac{P}{T}\right)+\sum_{i}^{c} n_{i} \mathrm{~d}\left(\frac{\mu_{i}}{T}\right),
$$

with canonical variables $\left\{1 / T, P / T,\left\{\mu_{i} / T\right\}\right\}$. This property of the complete Legendre transform gives rise to the entropy-based Gibbs-Duhem equation, which shows that the intensive parameters characterizing the system, i.e., $1 / T, P / T$ and $\mu_{i} / T$, are not independent of each other. 
As shown in Eq. 40, a partial Legendre transform $f^{(p)}$ of order $p$ of the base function $f^{(0)}\left(X_{1}, X_{2}, \ldots, X_{n}\right)$, with $1 \leq p \leq(n-1)$, is obtained via subtraction of $p$ products of $X_{i}$ with its conjugate partial derivative $c_{i}=\left(\partial f^{(0)} / \partial X_{i}\right)_{X_{j \neq i}}$, i.e., via subtraction of $\sum_{i=1}^{p} c_{i} X_{i}$. The number of partial Legendre transforms of order $p$ is therefore given by the number of combinations without repetition, that is, by

$$
C_{p}^{n}=\frac{n !}{p !(n-p) !},
$$

The total number $N_{\mathrm{Le}, p}$ of partial Legendre transforms, that is, the total number of equivalent alternatives to $f^{(0)}$, is thus obtained from

$$
N_{\text {Le }, p}=\sum_{p=1}^{p=n-1} C_{p}^{n}=2^{n}-2 .
$$

Since the total number $N_{\mathrm{Le}}^{\mathrm{t}}$ of Legendre transforms includes the complete transform, it is given by

$$
N_{\mathrm{Le}}^{\mathrm{t}}=\sum_{p=1}^{p=n} C_{p}^{n}=N_{\mathrm{Le}, p}+1=2^{n}-1 .
$$

The complete Legendre transform, that is, the transform of order $p=n$, vanishes identically.

Application of the above results to the fundamental equations for an open multicomponent PVTx phase either in the internal energy representation, Eq. 15, or in the entropy representation, Eq. 16, is now straightforward. Provided the summation term $\sum_{i} \mu_{i} n_{i}$ in the former is treated as a single term (in this case $n=3$ ), the entire number $N^{\mathrm{t}}$ of equivalent primary functions (equivalent thermodynamic potentials) related to the internal energy, including $n U$, and therefore the number of the corresponding equivalent fundamental property relations, i.e., of the total differentials of these functions, is seven:

$$
N^{\mathrm{t}}=N_{\mathrm{Le}, p}+1=2^{n}-1=7,
$$

They are presented in Tables 1 and 2, respectively, together with the null function and its associated internal energy-based Gibbs-Duhem equation [37].

Since the total differentials of the primary functions presented in Table 2 are equivalent, alternatives to the definition of the chemical potential $\mu_{i}$ of component $i$ by Eq. 11 are possible:

$$
\begin{aligned}
\mu_{i} & \equiv\left[\frac{\partial(n U)}{\partial n_{i}}\right]_{n S, n V, n_{j \neq i}}=\left[\frac{\partial(n H)}{\partial n_{i}}\right]_{n S, P, n_{j \neq i}} \\
& =\left[\frac{\partial(n F)}{\partial n_{i}}\right]_{T, n V, n_{j \neq i}}=\left[\frac{\partial(n G)}{\partial n_{i}}\right]_{T, P, n_{j \neq i}} .
\end{aligned}
$$

The last equality, that is, 
Table 1 Equivalent alternative extensive primary functions (thermodynamic potentials) related to the extensive internal energy $n U$ (see Eq. 15), applying to single-phase, multicomponent PVTx systems, either open or closed

\begin{tabular}{|c|c|c|c|}
\hline \multirow[t]{2}{*}{ Primary form } & \multicolumn{2}{|c|}{$\begin{array}{l}\text { Primary function (thermodynamic poten- } \\
\text { tial) }\end{array}$} & \multirow[t]{2}{*}{ Alternative form } \\
\hline & Symbol & Name & \\
\hline$n U$ & $n U$ & Internal energy & $T(n S)-P(n V)+\sum_{i}^{c} \mu_{i} n_{i}$ \\
\hline$n U+P(n V)$ & $n H$ & Enthalpy & $T(n S)+\sum_{i}^{c} \mu_{i} n_{i}$ \\
\hline$n U-T(n S)$ & $n F$ & Helmholtz energy & $-P(n V)+\sum_{i}^{c} \mu_{i} n_{i}$ \\
\hline $\left.\begin{array}{l}n U-T(n S)+P(n V) \\
=n H-T(n S)=n F+P(n V)\end{array}\right\}$ & $n G$ & Gibbs energy & $\sum_{i}^{c} \mu_{i} n_{i}$ \\
\hline$n U-\sum_{i}^{c} \mu_{i} n_{i}$ & $n X$ & Not named & $T(n S)-P(n V)$ \\
\hline $\left.\begin{array}{l}n U+P(n V)-\sum_{i}^{c} \mu_{i} n_{i} \\
=n H-\sum_{i}^{c} \mu_{i} n_{i}\end{array}\right\}$ & $n Y$ & Not named & $T(n S)$ \\
\hline $\left.\begin{array}{l}n U-T(n S)-\sum_{i}^{c} \mu_{i} n_{i} \\
=n F-\sum_{i}^{c} \mu_{i} n_{i}\end{array}\right\}$ & $n J$ & Grand canonical potential & $-P(n V)$ \\
\hline $\left.\begin{array}{l}n U-T(n S)+P(n V)-\sum_{i}^{c} \mu_{i} n_{i} \\
=n G-\sum_{i}^{c} \mu_{i} n_{i}\end{array}\right\}$ & & Null function & 0 \\
\hline
\end{tabular}

They are obtained via Legendre transformations of the fundamental equation in the internal energy representation. Also listed is the complete Legendre transform that vanishes identically, i.e., the null function

$$
\begin{aligned}
\mu_{i}\left(T, P, x_{1}, x_{2}, \ldots\right) & \equiv\left[\frac{\partial(n G)}{\partial n_{i}}\right]_{T, P, n_{j \neq i}} \\
& \equiv G_{i}\left(T, P, x_{1}, x_{2}, \ldots\right)
\end{aligned}
$$

is the preferred working definition, because $T$ and $P$ are the most useful experimental thermodynamic coordinates. Recalling the definition of a partial molar property, that is,

$$
M_{i} \equiv\left(\frac{\partial(n M)}{\partial n_{i}}\right)_{T, P, n_{j \neq i}}, n=\sum_{i} n_{i},
$$


Table 2 Equivalent alternative forms of the fundamental property relation in the internal energy representation (see Eq. 13)
Total (exact) differential of the primary function (Total (exact) differential of the thermodynamic potential)

\begin{tabular}{ll}
\hline $\mathrm{d}(n U)=T \mathrm{~d}(n S)-P \mathrm{~d}(n V)+\sum_{i}^{c} \mu_{i} \mathrm{~d} n_{i}$ & $n S, n V,\left\{n_{i}\right\}$ \\
$\mathrm{d}(n H)=T \mathrm{~d}(n S)+(n V) \mathrm{d} P+\sum_{i}^{c} \mu_{i} \mathrm{~d} n_{i}$ & $n S, P,\left\{n_{i}\right\}$ \\
$\mathrm{d}(n F)=-(n S) \mathrm{d} T-P \mathrm{~d}(n V)+\sum_{i}^{c} \mu_{i} \mathrm{~d} n_{i}$ & $T, n V,\left\{n_{i}\right\}$ \\
$\mathrm{d}(n G)=-(n S) \mathrm{d} T+(n V) \mathrm{d} P+\sum_{i}^{c} \mu_{i} \mathrm{~d} n_{i}$ & $T, P,\left\{n_{i}\right\}$ \\
$\mathrm{d}(n X)=T \mathrm{~d}(n S)-P \mathrm{~d}(n V)-\sum_{i}^{c} n_{i} \mathrm{~d} \mu_{i}$ & $n S, n V,\left\{\mu_{i}\right\}$ \\
$\mathrm{d}(n Y)=T \mathrm{~d}(n S)+(n V) \mathrm{d} P-\sum_{i}^{c} n_{i} \mathrm{~d} \mu_{i}$ & $n S, P,\left\{\mu_{i}\right\}$ \\
$\mathrm{d}(n J)=-(n S) \mathrm{d} T-P \mathrm{~d}(n V)-\sum_{i}^{c} n_{i} \mathrm{~d} \mu_{i}$ & $T, n V,\left\{\mu_{i}\right\}$ \\
$0=-(n S) \mathrm{d} T+(n V) \mathrm{d} P-\sum_{i}^{c} n_{i} \mathrm{~d} \mu_{i}$ & $T, P,\left\{\mu_{i}\right\}$
\end{tabular}

They are total (exact) differentials of the primary functions (thermodynamic potentials) presented in Table 1, and thus apply to single-phase, multicomponent PVTx systems either open or closed. Also listed is the Gibbs-Duhem equation corresponding to the null function

where $n M=M^{\mathrm{t}}\left(T, P, n_{1}, n_{2}, \ldots\right)$ is an extensive mixture thermodynamic property, and $M=M\left(T, P, x_{1}, x_{2}, \ldots\right)$ stands for any intensive thermodynamic mixture property (excluding, of course, temperature, pressure and composition), we recognize that $\mu_{i}$ is the partial molar Gibbs energy $G_{i}$ of component $i$ in solution. Note that a partial molar property $M_{i}\left(T, P, x_{1}, x_{2}, \ldots\right)$ is an intensive state function, a property of the mixture, and in general $M_{i} \neq M_{i}^{*} ; M_{i}$ s obeys the summability relations:

$$
M=\sum_{i} x_{i} M_{i} \text { or } n M=\sum_{i} n_{i} M_{i}
$$

respectively.

Analogically, when treating the summation term $\sum_{i}^{c} \frac{\mu_{i}}{T} n_{i}$ in Eq. 16 (entropy representation) as a single term (in this case again $n=3$ ), the entire number of equivalent primary functions (equivalent thermodynamic potentials) related to the entropy, including $n S$, and therefore the number of corresponding equivalent fundamental property relations is also seven. They are summarized in Tables 3 and 4, respectively, together with the appropriate null function and its associated entropy-based Gibbs-Duhem equation [37]. The replacement of one or more of the extensive variables $n U, n V,\left\{n_{i}\right\}$ by the corresponding conjugate intensive variable(s) $1 / T, P / T$ and $\mu_{i} / T$, respectively, yields primary functions known as Massieu-Planck functions. Interestingly, such a Legendre transform of the entropy was already reported by Massieu in 1869, and thus predates the Legendre transforms of the internal energy reported by Gibbs in 1875 (see Callen [30]). 
Table 3 Equivalent alternative extensive primary functions (thermodynamic potentials) related to the extensive entropy $n S$ (see Eq. 16), applying to single-phase, multicomponent PVTx systems, either open or closed

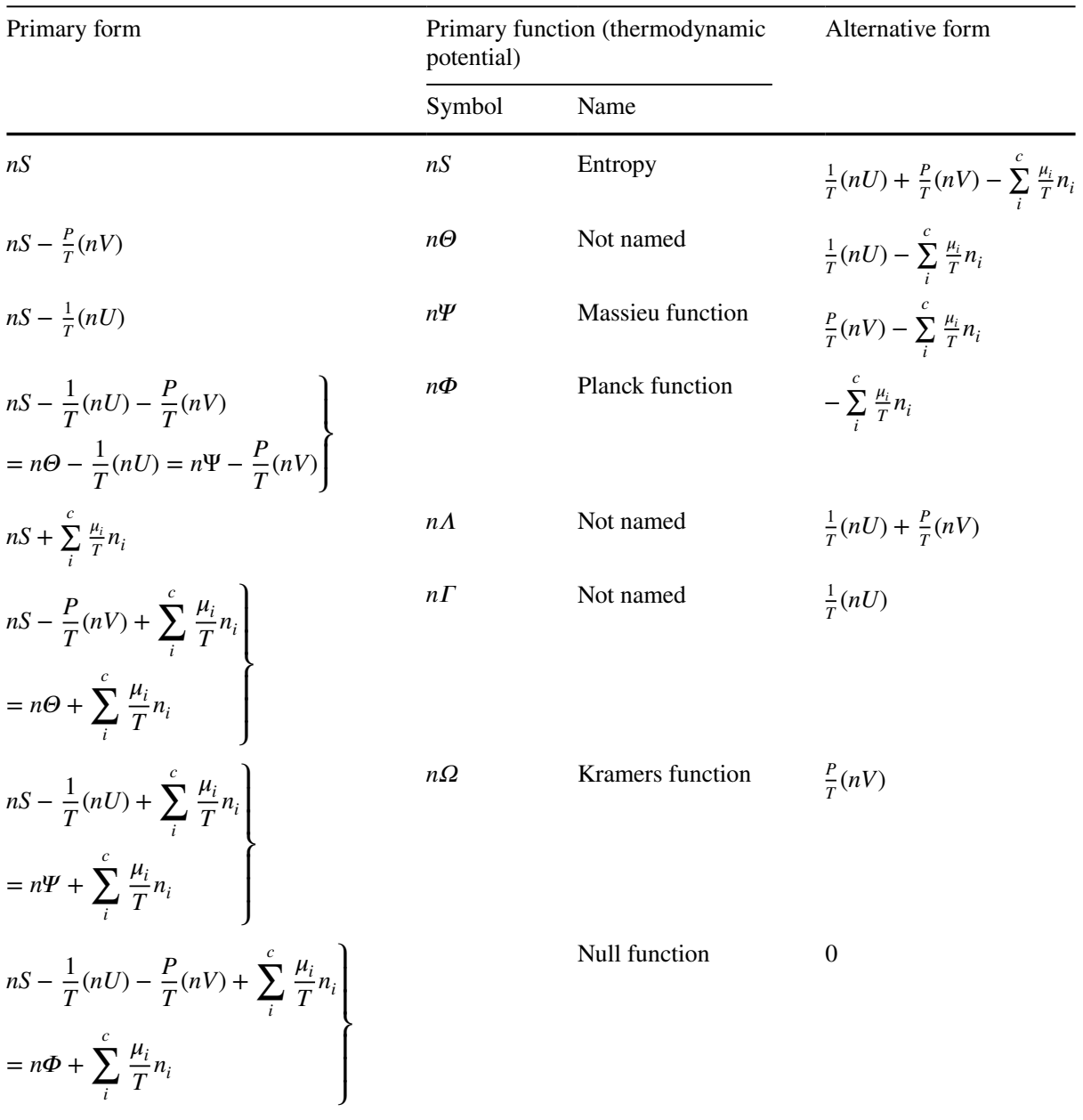

They are obtained via Legendre transformation of the fundamental equation in the entropy representation and are known as Massieu-Planck functions. Also listed is the complete Legendre transform that vanishes identically, i.e., the null function

Though not always immediately recognized, the (molar) Massieu-Planck functions are simply related to the (molar) thermodynamic potentials [37]:

$$
\begin{gathered}
S=\frac{Y}{T}, \\
\Theta=\frac{T S-P V}{T}=\frac{X}{T},
\end{gathered}
$$


Table 4 Equivalent alternative forms of the fundamental property relation in the entropy representation (see Eq. 14)
Total (exact) differential of the primary function (Total (exact) differential of the thermodynamic potential)

\begin{tabular}{ll}
\hline $\mathrm{d}(n S)=\frac{1}{T} \mathrm{~d}(n U)+\frac{P}{T} \mathrm{~d}(n V)-\sum_{i}^{c} \frac{\mu_{i}}{T} \mathrm{~d} n_{i}$ & $n U, n V,\left\{n_{i}\right\}$ \\
$\mathrm{d}(n \Theta)=\frac{1}{T} \mathrm{~d}(n U)-(n V) \mathrm{d} \frac{P}{T}-\sum_{i}^{c} \frac{\mu_{i}}{T} \mathrm{~d} n_{i}$ & $n U, \frac{P}{T},\left\{n_{i}\right\}$ \\
$\mathrm{d}(n \Psi)=-(n U) \mathrm{d} \frac{1}{T}+\frac{P}{T} \mathrm{~d}(n V)-\sum_{i}^{c} \frac{\mu_{i}}{T} \mathrm{~d} n_{i}$ & $\frac{1}{T}, n V,\left\{n_{i}\right\}$ \\
$\mathrm{d}(n \Phi)=-(n U) \mathrm{d} \frac{1}{T}-(n V) \mathrm{d} \frac{P}{T}-\sum_{i}^{c} \frac{\mu_{i}}{T} \mathrm{~d} n_{i}$ & $\frac{1}{T}, \frac{P}{T},\left\{n_{i}\right\}$ \\
$\mathrm{d}(n \Lambda)=\frac{1}{T} \mathrm{~d}(n U)+\frac{P}{T} \mathrm{~d}(n V)+\sum_{i}^{c} n_{i} \mathrm{~d} \frac{\mu_{i}}{T}$ & $n U, n V,\left\{\frac{\mu_{i}}{T}\right\}$ \\
$\mathrm{d}(n \Gamma)=\frac{1}{T} \mathrm{~d}(n U)-(n V) \mathrm{d} \frac{P}{T}+\sum_{i}^{c} n_{i} \mathrm{~d} \frac{\mu_{i}}{T}$ & $n U, \frac{P}{T},\left\{\frac{\mu_{i}}{T}\right\}$ \\
$\mathrm{d}(n \Omega)=-(n U) \mathrm{d} \frac{1}{T}+\frac{P}{T} \mathrm{~d}(n V)+\sum_{i}^{c} n_{i} \mathrm{~d} \frac{\mu_{i}}{T}$ & $\frac{1}{T}, n V,\left\{\frac{\mu_{i}}{T}\right\}$ \\
$0=-(n U) \mathrm{d} \frac{1}{T}-(n V) \mathrm{d} \frac{P}{T}+\sum_{i}^{c} n_{i} \mathrm{~d} \frac{\mu_{i}}{T}$ & $\frac{1}{T}, \frac{P}{T},\left\{\frac{\mu_{i}}{T}\right\}$
\end{tabular}

They represent total (exact) differentials of the primary functions (thermodynamic potentials) presented in Table 3, and thus apply to single-phase, multicomponent PVTx systems, either open or closed. They are known as fundamental Massieu-Planck property relations. Also listed is the Gibbs-Duhem equation corresponding to the null function

$$
\begin{gathered}
\Psi=\frac{T S-U}{T}=-\frac{F}{T}, \\
\Phi=\frac{T S-U-P V}{T}=-\frac{G}{T}, \\
\Lambda=\frac{U+P V}{T}=\frac{H}{T}, \\
\Omega=\frac{P}{T} V=-\frac{U}{T},
\end{gathered}
$$

At constant composition, the fundamental property relations corresponding to Legendre transforms excluding the chemical potentials are readily obtained, and for one mole of a homogeneous fluid at constant composition, the following four energy-based fundamental property relations apply:

$$
\mathrm{d} U(S, V)=T \mathrm{~d} S-P \mathrm{~d} V,
$$




$$
\begin{gathered}
\mathrm{d} H(S, P)=T \mathrm{~d} S+V \mathrm{~d} P, \\
\mathrm{~d} F(T, V)=-S \mathrm{~d} T-P \mathrm{dV}, \\
\mathrm{d} G(T, P)=-S \mathrm{~d} T+V \mathrm{~d} P .
\end{gathered}
$$

Focusing now on the molar Helmholtz energy and the molar Gibbs energy, Eqs. 62 and 63 yield

$$
S=-(\partial F / \partial T)_{V}=-(\partial G / \partial T)_{P},
$$

and in view of the definitions of $F$ and $G$ (see Table 1) and Eq. 64, the Gibbs-Helmholtz equations

$$
U=F-T(\partial F / \partial T)_{V},
$$

and

$$
H=G-T(\partial G / \partial T)_{P},
$$

are obtained. For simplicity's sake the subscript $\left\{x_{i}\right\}$, indicating constant composition, has been omitted. Simple mathematical manipulations yield the following alternative forms:

$$
\left(\frac{\partial(F / T)}{\partial T}\right)_{V}=-\frac{U}{T^{2}}
$$

and

$$
\left(\frac{\partial(G / T)}{\partial T}\right)_{P}=-\frac{H}{T^{2}} .
$$

Equation 68 suggests an alternative to the fundamental property relation involving the Gibbs energy as presented in Table 2 i.e.,

$$
\mathrm{d}(n G)=-(n S) \mathrm{d} T+(n V) \mathrm{d} P+\sum_{i}^{c} \mu_{i} \mathrm{~d} n_{i},
$$

By introducing the dimensionless property $G / R T$,

$$
\mathrm{d}\left(\frac{n G}{R T}\right)=-\frac{n H}{R T^{2}} \mathrm{~d} T+\frac{n V}{R T} \mathrm{~d} P+\sum_{i}^{c} \frac{\mu_{i}}{R T} \mathrm{~d} n_{i},
$$

is obtained, and

$$
\frac{G}{R T}=\sum_{i}^{c} x_{i} \frac{\mu_{i}}{R T}=\sum_{i}^{c} x_{i} \frac{G_{i}}{R T} .
$$

Equation 70 is of considerable utility. All terms have the dimension of amount-of-substance and, in contradistinction to Eq. 69, the enthalpy rather than the entropy appears in the first term of the right-hand side of this exact differential, with obvious benefits for the discussion of experimental results. 
Analogously, Eq. 67 suggests an alternative to the fundamental property relation involving the Helmholtz energy, $F$, as presented in Table 2 i.e.,

$$
\mathrm{d}(n F)=-(n S) \mathrm{d} T-P \mathrm{~d}(n V)+\sum_{i}^{c} \mu_{i} \mathrm{~d} n_{i} .
$$

By introducing the dimensionless property $F / R T$, the corresponding fundamental property relation reads

$$
\mathrm{d}\left(\frac{n F}{R T}\right)=-\frac{n U}{R T^{2}} \mathrm{~d} T-\frac{P}{R T} \mathrm{~d}(n V)+\sum_{i}^{c} \frac{\mu_{i}}{R T} \mathrm{~d} n_{i},
$$

with

$$
\frac{F}{R T}=-\frac{P V}{R T}+\sum_{i}^{c} x_{i} \frac{\mu_{i}}{R T} .
$$

Note, that in contradistinction to Eq. 72, the first term on the right-hand side of Eq. 73 contains the internal energy instead of the entropy.

\section{Thermodynamic Properties of Fluids}

\subsection{Residual Properties}

The thermodynamic equations formally introduced in Sect. 1.2 establish exact relations between system properties and judiciously selected variables, the most convenient being the sets $\left\{T, P,\left\{x_{i}\right\}\right\}$ and $\left\{T, V,\left\{x_{i}\right\}\right\}$. However, they do not provide numerical values for any thermodynamic property: reliable experimental data and reliable models are necessary to reach this goal. The perfect gas [29] (or ideal gas), identified by the superscript pg, is a hypothetical substance for which the intermolecular potential energy is zero, and which obeys (see Eq. 6) the simple PVT equation of state

$$
P V^{\mathrm{pg}}=R T \text {. }
$$

While no real fluid conforms to this model, nevertheless the concept is eminently useful, because (a) the associated simple equations may frequently be used as reasonable approximations of real-gas behavior at low pressures, and (b) the model serves as the basis for the definition of extremely useful auxiliary functions known as residual functions, which play a central role in the description of real-fluid behavior. Note, that in the limit of vanishingly small pressure, real-gas behavior in many respects approaches perfect-gas behavior, but not in all, a fact which is frequently overlooked. Specifically, for a constant composition gas (this includes a pure gas) at constant temperature,

$$
\lim _{P \rightarrow 0} Z=1 \text {. }
$$

For comparing real-fluid properties to perfect-gas properties at the same temperature, same pressure and same composition, say, the actual molar volume $V$ to $V^{\mathrm{pg}}$, two obvious choices exist: one may quantify deviations in terms of a ratio measure, here the compression factor 


$$
Z \equiv V / V^{\mathrm{pg}}=P V / R T
$$

or in terms of a difference measure, here the molar residual volume,

$$
V^{\mathrm{R}} \equiv V-V^{\mathrm{pg}}=V-R T / P .
$$

The two functions are, of course, related,

$$
V^{\mathrm{R}}=R T \frac{Z-1}{P}
$$

and since the zero-pressure limit of $V^{\mathrm{R}}$ becomes indeterminate, that is, both the numerator $(Z-1)$ and the denominator $P$ vanish as the pressure reaches its limiting value zero, de l'Hôpital's rule yields

$$
\lim _{P \rightarrow 0} V^{\mathrm{R}}=R T \lim _{P \rightarrow 0}\left(\frac{\partial Z}{\partial P}\right)_{T}
$$

Since experiments show that $(\partial Z / \partial P)_{T}$ remains generally finite in the limit of vanishingly small pressure, $V^{\mathrm{R}}$ remains also generally finite; it is given by the second amount density-series virial coefficient $B$,

$$
\lim _{P \rightarrow 0} V^{\mathrm{R}}=B .
$$

Hence $V^{\mathrm{R}}$ is nonzero except at the Boyle temperature at which $B=0$. Equation 81 identifies an experimentally accessible macroscopic property as a key thermophysical quantity establishing an important link to the intermolecular pair-potential energy function $u(r)$ [41-43]:

$$
B=-2 \pi N_{\mathrm{A}} \int_{0}^{\infty}\left[\exp \left(-u(r) / k_{\mathrm{B}} T\right)-1\right] r^{2} \mathrm{~d} r .
$$

Here, $N_{\mathrm{A}}$ is the Avogadro constant, $k_{\mathrm{B}}$ is the Boltzmann constant, ${ }^{2}$ and $N_{\mathrm{A}} k_{\mathrm{B}}=R$. Note, however, that Eq. 82 is for pair-potential energies that do not depend on the relative orientation of the molecules.

In analogy to $V^{\mathrm{R}}$, molar isobaric residual properties $M^{\mathrm{R}}$ of a single-phase pure fluid or constant-composition fluid mixture are defined similarly by [17, 18, 29, 46]

$$
M^{\mathrm{R}}\left(T, P,\left\{x_{i}\right\}\right) \equiv M\left(T, P,\left\{x_{i}\right\}\right)-M^{\mathrm{pg}}\left(T, P,\left\{x_{i}\right\}\right),
$$

where the $M \mathrm{~s}$ denote molar values of any extensive thermodynamic property $n M\left(T, P,\left\{x_{i}\right\}\right)$, such as $U, H, S, V, G$ or $F . M\left(T, P,\left\{x_{i}\right\}\right)$ is the actual molar property value of the fluid at the temperature, pressure and composition of interest, and $M^{\mathrm{pg}}\left(T, P,\left\{x_{i}\right\}\right)$ is the molar property value for the fluid in its perfect-gas state at the same T, $P$ and $\left\{x_{i}\right\}$. Note that the perfect-gas state is hypothetical except in the zero-pressure limit, where the perfect-gas EOS is valid (that is, for this real perfect-gas state $P V=R T$ ). Residual properties

\footnotetext{
${ }^{2}$ With the Avogadro constant $N_{\mathrm{A}}$ and the Boltzmann constant $k_{\mathrm{B}}$ now being exactly defined [44, 45], that is, $N_{\mathrm{A}}=6.02214076 \times 10^{23} \mathrm{~mol}^{-1}$ and $k_{\mathrm{B}}=1.380649 \times 10^{-23} \mathrm{~J} \mathrm{~K}^{-1}$, the (molar) gas constant is $R=N_{\mathrm{A}} k_{\mathrm{B}}=8.314462618 \mathrm{~J} \mathrm{~K}^{-1} \mathrm{~mol}^{-1}$.
} 
are the most direct measures of the effects of the intermolecular forces. The computation of values $M$ of any thermodynamic fluid property is based on

$$
M\left(T, P,\left\{x_{i}\right\}\right)=M^{\mathrm{R}}\left(T, P,\left\{x_{i}\right\}\right)+M^{\mathrm{pg}}\left(T, P,\left\{x_{i}\right\}\right),
$$

From the defining equation, Eq. 83, we have for a differential change in state at constant $T$ and constant $\left\{x_{i}\right\}$

$$
\mathrm{d} M^{\mathrm{R}}=\left[\left(\frac{\partial M}{\partial P}\right)_{T,\left\{x_{i}\right\}}-\left(\frac{\partial M^{\mathrm{pg}}}{\partial P}\right)_{T,\left\{x_{i}\right\}}\right] \mathrm{d} P,
$$

and integration from $P=0$ to the pressure of interest $P$ yields

$$
\begin{aligned}
M^{\mathrm{R}}\left(T, P,\left\{x_{i}\right\}\right)= & \left(M^{\mathrm{R}}\right)_{\text {zero pressure }} \\
& +\int_{0}^{P}\left[\left(\frac{\partial M}{\partial P}\right)_{T,\left\{x_{i}\right\}}-\left(\frac{\partial M^{\mathrm{pg}}}{\partial P}\right)_{T,\left\{x_{i}\right\}}\right] \mathrm{d} P \text {, constant } T,\left\{x_{i}\right\} .
\end{aligned}
$$

In contradistinction to the observed limiting behavior of the residual volume, see Eq. 81, experimental evidence indicates that for the pivotal properties enthalpy and entropy the zero-pressure terms can be set equal to zero (at constant $T$ and $\left\{x_{i}\right\}$ ), that is,

$$
\left.\begin{array}{l}
\lim _{P \rightarrow 0} M^{\mathrm{R}}\left(T, P,\left\{x_{i}\right\}\right)=\left(M^{\mathrm{R}}\right)_{\text {zero pressure }}=0 \\
\text { for } \\
M^{\mathrm{R}}=H^{\mathrm{R}}\left(T, P,\left\{x_{i}\right\}\right) \text { and } M^{\mathrm{R}}=S^{\mathrm{R}}\left(T, P,\left\{x_{i}\right\}\right)
\end{array}\right\} .
$$

Thus, for the molar isobaric residual enthalpy $H^{\mathrm{R}}\left(T, P,\left\{x_{i}\right\}\right)$ we obtain, in conjunction with $(\partial H / \partial P)_{T,\left\{x_{i}\right\}}=V-T(\partial V / \partial T)_{P,\left\{x_{i}\right\}},\left(\partial H^{\mathrm{pg}} / \partial P\right)_{T,\left\{x_{i}\right\}}=0$, and Eq. 87

$$
H^{\mathrm{R}}\left(T, P,\left\{x_{i}\right\}\right)=\int_{0}^{P}\left[V-T\left(\frac{\partial V}{\partial T}\right)_{P,\left\{x_{i}\right\}}\right] \mathrm{d} P=-R T^{2} \int_{0}^{P}\left(\frac{\partial Z}{\partial T}\right)_{P,\left\{x_{i}\right\}} \frac{\mathrm{d} P}{P}, \text { constant } T,\left\{x_{i}\right\},
$$

and for the molar isobaric residual entropy, we obtain, in conjunction with $(\partial S / \partial P)_{T,\left\{x_{i}\right\}}=-(\partial V / \partial T)_{P,\left\{x_{i}\right\}},\left(\partial S^{\mathrm{pg}} / \partial P\right)_{T,\left\{x_{i}\right\}}=-R / P$, and Eq. 87,

$$
\begin{aligned}
S^{\mathrm{R}}\left(T, P,\left\{x_{i}\right\}\right) & =\int_{0}^{P}\left[-\left(\frac{\partial V}{\partial T}\right)_{P,\left\{x_{i}\right\}}+\frac{R}{P}\right] \mathrm{d} P \\
& =-R \int_{0}^{P}\left[T\left(\frac{\partial Z}{\partial T}\right)_{P,\left\{x_{i}\right\}}+Z-1\right] \frac{\mathrm{d} P}{P}, \text { constant } T,\left\{x_{i}\right\} .
\end{aligned}
$$

Since Eq. 87 is also valid for $M=C_{P}$, and the pressure dependence of the molar isobaric heat capacity $C_{p}=(\partial H / \partial T)_{P,\left\{x_{i}\right.}$ is given by

$$
\left(\frac{\partial C_{P}}{\partial P}\right)_{T,\left\{x_{i}\right\}}=-T\left(\frac{\partial^{2} V}{\partial T^{2}}\right)_{P,\left\{x_{i}\right\}},
$$


and $\left(\partial C_{P}^{\mathrm{pg}} / \partial P\right)_{T,\left\{x_{i}\right\}}=0$, the molar isobaric residual constant-pressure heat capacity is obtained from

$$
\begin{aligned}
& C_{P}^{\mathrm{R}}\left(T, P,\left\{x_{i}\right\}\right)=-T \int_{0}^{P}\left(\frac{\partial^{2} V}{\partial T^{2}}\right)_{P,\left\{x_{i}\right\}} \\
& \left.\mathrm{d} P=-R T \int_{0}^{P}\left[T\left(\frac{\partial^{2} Z}{\partial T^{2}}\right)_{P,\left\{x_{i}\right\}}+2\left(\frac{\partial Z}{\partial T}\right)_{P,\left\{x_{i}\right\}}\right] \frac{\mathrm{d} P}{P}\right\} \text { constant } T,\left\{x_{i}\right\} .
\end{aligned}
$$

Clearly,

$$
\left.\begin{array}{l}
U^{\mathrm{R}}=H^{\mathrm{R}}-P V^{\mathrm{R}}=H^{\mathrm{R}}-R T(Z-1), \\
G^{\mathrm{R}}=H^{\mathrm{R}}-T S^{\mathrm{R}}=R T \int_{0}(Z-1) \frac{\mathrm{d} P}{P}, \\
F^{\mathrm{R}}=U^{\mathrm{R}}-T S^{\mathrm{R}}=G^{\mathrm{R}}-R T(Z-1),
\end{array}\right\}
$$

and all three functions approach zero for $P \rightarrow 0$. The isobaric residual functions are the conventional forms since they are advantageously based on $\left\{T, P,\left\{x_{i}\right\}\right\}$ as independent variables. They have been most useful in applications to real gases and gas mixtures, though their suitability in dealing with liquid systems has been greatly furthered by the application of the corresponding-states theorem (CST): this theorem is firmly based on statistical mechanics and occupies a leading position in the field of property estimation [1, 16-18, 36, 47-50]. The most popular approaches are based on Pitzer's three-parameter CST correlation [51-55]. For pure (*) fluids, that is, for gases, vapors and liquids, the compression factor $Z^{*}=P V^{*} / R T$ is expressed as a function of reduced temperature $T_{\mathrm{r}} \equiv T / T_{\mathrm{c}}$, reduced pressure $P_{\mathrm{r}} \equiv P / P_{\mathrm{c}}$ and acentric factor $\omega$, which quantity is defined by

$$
\omega \equiv-1-\log _{10}\left(P_{\sigma, \mathrm{r}}\right)_{T_{\mathrm{r}}=0.7} .
$$

Here, $P_{\sigma, \mathrm{r}} \equiv P_{\sigma} / P_{\mathrm{c}}$ is the reduced vapor pressure $P_{\sigma}(T)$ at reduced temperature $T_{\mathrm{r}}=0.7$. Specifically, in the key three-parameter CST correlation

$$
\begin{aligned}
Z^{*} & =\frac{P V^{*}}{R T}=f\left(T_{\mathrm{r}}, P_{\mathrm{r}}, \omega\right) \\
& =Z^{(0)}\left(T_{\mathrm{r}}, P_{\mathrm{r}}\right)+\omega Z^{(1)}\left(T_{\mathrm{r}}, P_{\mathrm{r}}\right), \text { all fluids, }
\end{aligned}
$$

$Z^{(0)}$ represents the simple-fluid contribution to $Z^{*}$ that is based on experimental PVT data of $\mathrm{Ar}, \mathrm{Kr}$ and $\mathrm{Xe}$ for which $\omega$ is essentially zero (and thus a two-parameter CST correlation suffices); $Z^{(1)}$ represents the non-simple-fluid contribution to $Z^{*}$ : it is determined via experimental $P V T$ data of selected fluids with $\omega \neq 0$ (quantum fluids, strongly polar fluids and fluids with strong hydrogen bonds are excluded). Critically evaluated values of the $\omega \mathrm{s}$ for many fluids, together with values for $T_{\mathrm{c}}$ and $P_{\mathrm{c}}$, are tabulated in Ref. 49. One of the best Pitzer-type correlations is that developed by Lee and Kesler [56-58]: these authors present tables for the contributions $Z^{(0)}\left(T_{\mathrm{r}}, P_{\mathrm{r}}\right)$ and $Z^{(1)}\left(T_{\mathrm{r}}, P_{\mathrm{r}}\right)$, as well as for derived functions 
for both liquid and vapor phases, covering large temperature and pressure ranges, i.e., $0.30 \leq T_{\mathrm{r}} \leq 4.00$ and $0.01 \leq P_{\mathrm{r}} \leq 10.00$.

In order to use generalized CST correlations for mixtures, conventional practice is based on the assumption that mixture properties can be represented by the same correlation types developed for pure fluids, though with appropriately defined values for the corresponding-states scaling parameters of the mixture, that is, by essentially empirically averaging pure-component parameters $T_{\mathrm{c}, i}, P_{\mathrm{c}, i}$ and $\omega_{i}$ to obtain pseudocritical temperatures $T_{\mathrm{pc}}$, pseudocritical pressures $P_{\mathrm{pc}}$ and pseudo-acentric factors $\omega_{\mathrm{p}}$ referring to the mixture. This is accomplished by using recipes known as mixing rules. Thus, a three-parameter CST correlation for the mixture compression factor $Z$, in the one-fluid approximation, may be written as

$$
Z=\frac{P V}{R T}=f\left(T_{\mathrm{pr}}, P_{\mathrm{pr}}, \omega_{\mathrm{p}}\right)=Z^{(0)}\left(T_{\mathrm{pr}}, P_{\mathrm{pr}}\right)+\omega_{\mathrm{p}} Z^{(1)}\left(T_{\mathrm{pr}}, P_{\mathrm{pr}}\right), \text { all fluids, }
$$

where the pseudoreduced temperature $T_{\mathrm{pr}}$ and the pseudoreduced pressure $P_{\mathrm{pr}}$ are defined by

$$
T_{\mathrm{pr}} \equiv T / T_{\mathrm{pc}} \text { and } P_{\mathrm{pr}} \equiv P / P_{\mathrm{pc}} .
$$

The simplest set of mixing rules for pseudocritical parameters are those of Kay [59]. They are defined as mole-fraction-weighted sums of the pure-component values, and so is $\omega_{\mathrm{p}}$, i.e.,

$$
T_{\mathrm{pc}}=\sum_{i}^{c} x_{i} T_{\mathrm{c}, i}, P_{\mathrm{pc}}=\sum_{i}^{c} x_{i} P_{\mathrm{c}, i}, \omega_{\mathrm{p}}=\sum_{i}^{c} x_{i} \omega_{i} .
$$

Although simple to apply, for mixtures of molecularly noticeably dissimilar fluids Kay's rules are often inadequate, and more flexible and thus more elaborate recipes must be introduced [49], such as quadratic mixing rules (reminiscent of those used in the multicomponent vdW model):

$$
T_{\mathrm{pc}}=\sum_{i}^{c} \sum_{j}^{c} x_{i} x_{j} T_{\mathrm{c}, i j} \text { and } P_{\mathrm{pc}}=\sum_{i}^{c} \sum_{j}^{c} x_{i} x_{j} P_{\mathrm{c}, i j},
$$

where $T_{\mathrm{c}, i i}$ and $T_{\mathrm{c}, j j}$ denote the critical temperatures of the pure components $i$ and $j$, respectively, $P_{\mathrm{c}, i i}$ and $P_{\mathrm{c}, j j}$ are their critical pressures, respectively, and for the evaluation of the cross parameters $T_{\mathrm{c}, i j}$ and $P_{\mathrm{c}, i j}$ empirical recipes known as combining rules are required.

However, I emphasize that temperature and volume, or alternatively, amount density $\rho_{n} \equiv 1 / V$, or number density, are the commonly used variables in statistical mechanics; and in addition, on the practical side, most PVTx equations of state, such as cubic vdW-type equations, are pressure-explicit [1, 16-18, 49, 60-65]: hence $T$ and $V$ (and for mixtures, of course, also the composition $\left\{x_{i}\right\}$ ) are the natural (canonical) independent variables. Thus, for a single-phase pure fluid or constant-composition mixture one may also define molar residual functions in $\left(T, V,\left\{x_{i}\right\}\right)$-space, i.e., molar isochoric (isometric) residual functions

$$
M^{\mathrm{r}}\left(T, V,\left\{x_{i}\right\}\right) \equiv M\left(T, V,\left\{x_{i}\right\}\right)-M^{\mathrm{pg}}\left(T, V,\left\{x_{i}\right\}\right)
$$

Again, the $M \mathrm{~s}$ denote molar properties of any extensive thermodynamic property $n M\left(T, n V,\left\{x_{i}\right\}\right)$ of the fluid, for instance, $U, H, S, G$ or $F$, and $M^{\mathrm{pg}}\left(T, V,\left\{x_{i}\right\}\right)$ is the corresponding molar property of the fluid in its hypothetical perfect-gas state at the same 
temperature, the same molar volume and the same composition. Note the important fact that if the temperature and the volume are the same for the real fluid state and the perfect gas state, the pressure is not the same. Equation 99 may also be used for the definition of a residual pressure, an intensive property:

$$
P^{\mathrm{r}}\left(T, V,\left\{x_{i}\right\}\right) \equiv P\left(T, V,\left\{x_{i}\right\}\right)-R T / V=R T \frac{Z-1}{V} .
$$

With the availability of a pressure-explicit EOS in the form $P=P\left(T, V,\left\{x_{i}\right\}\right)$, isochoric residual properties are the properties of direct interest. In complete analogy to Eqs. 86 and 87 we have

$$
\begin{aligned}
M^{\mathrm{r}}\left(T, V,\left\{x_{i}\right\}\right) & =\left(M^{\mathrm{r}}\right)_{\substack{\text { infinite volume } \\
\text { (zero pressure) }}} \\
& +\int_{\infty}^{V}\left[\left(\frac{\partial M}{\partial V}\right)_{T,\left\{x_{i}\right\}}-\left(\frac{\partial M^{\mathrm{pg}}}{\partial V}\right)_{T,\left\{x_{i}\right\}}\right] \mathrm{d} V, \text { constant } T,\left\{x_{i}\right\} .
\end{aligned}
$$

Again, experimental evidence indicates that for the pivotal properties internal energy and entropy the infinite-volume terms (zero-pressure terms) can be set equal to zero (at constant $T$ and $\left\{x_{i}\right\}$ ), that is,

$$
\left.\begin{array}{l}
\lim _{V \rightarrow \infty} M^{\mathrm{r}}\left(T, V,\left\{x_{i}\right\}\right)=\left(M^{\mathrm{r}}\right)_{\text {infinite volume }}=0 \\
\text { for } \\
M^{\mathrm{r}}=U^{\mathrm{r}}\left(T, V,\left\{x_{i}\right\}\right) \text { and } M^{\mathrm{r}}=S^{\mathrm{r}}\left(T, V,\left\{x_{i}\right\}\right)
\end{array}\right\} .
$$

Thus, for the molar isochoric residual internal energy $U^{\mathrm{r}}\left(T, V,\left\{x_{i}\right\}\right)$ we obtain, in conjunction with $(\partial U / \partial V)_{T,\left\{x_{i}\right\}}=-P+T(\partial P / \partial T)_{V,\left\{x_{i}\right\}},\left(\partial U^{\mathrm{pg}} / \partial V\right)_{T,\left\{x_{i}\right\}}=0$, and Eq. 102,

$$
U^{\mathrm{r}}\left(T, V,\left\{x_{i}\right\}\right)=\int_{\infty}^{V}\left[-P+T\left(\frac{\partial P}{\partial T}\right)_{V,\left\{x_{i}\right\}}\right] \mathrm{d} V=R T^{2} \int_{\infty}^{V}\left(\frac{\partial Z}{\partial T}\right)_{V,\left\{x_{i}\right\}} \frac{\mathrm{d} V}{V}, \text { constant } T,\left\{x_{i}\right\},
$$

and for the molar isochoric residual enthalpy $H^{\mathrm{r}}\left(T, V,\left\{x_{i}\right\}\right)$ we obtain, in conjunction with $(\partial H / \partial V)_{T,\left\{x_{i}\right\}}=T(\partial P / \partial T)_{V,\left\{x_{i}\right\}}+V(\partial P / \partial V)_{T,\left\{x_{i}\right\}},\left(\partial H^{\mathrm{pg}} / \partial V\right)_{T,\left\{x_{i}\right\}}=0$, and Eq. 87, at constant temperature and composition

$$
\left.H^{\mathrm{r}}\left(T, V,\left\{x_{i}\right\}\right)=\int_{\infty}^{V}\left[T\left(\frac{\partial P}{\partial T}\right)_{V,\left\{x_{i}\right\}}+V\left(\frac{\partial P}{\partial V}\right)_{T,\left\{x_{i}\right\}}\right] \mathrm{d} V=R T^{2} \int_{\infty}^{V}\left(\frac{\partial Z}{\partial T}\right)_{V,\left\{x_{i}\right\}} \frac{\mathrm{d} V}{V}+R T(Z-1)\right\} .
$$

For the molar isochoric residual entropy $S^{\mathrm{r}}\left(T, V,\left\{x_{i}\right\}\right)$ we obtain, in conjunction with $(\partial S / \partial V)_{T,\left\{x_{i}\right\}}=(\partial P / \partial T)_{V,\left\{x_{i}\right\}},\left(\partial S^{\mathrm{pg}} / \partial V\right)_{T,\left\{x_{i}\right\}}=R / V$, and Eq. 101, at constant temperature and composition

$$
S^{\mathrm{r}}\left(T, V,\left\{x_{i}\right\}\right)=\int_{\infty}^{V}\left[\left(\frac{\partial P}{\partial T}\right)_{V,\left\{x_{i}\right\}}-\frac{R}{V}\right] \mathrm{d} V=R \int_{\infty}^{V}\left[T\left(\frac{\partial Z}{\partial T}\right)_{V,\left\{x_{i}\right\}}+Z-1\right] \frac{\mathrm{d} V}{V} .
$$


Since $C_{V}$ of a real gas approaches $C_{V}^{\mathrm{pg}}$ for $V \rightarrow \infty$ (i.e., for $P \rightarrow 0$ ), Eqs. 101 and 102 apply, and together with $\left(\partial C_{V}^{\mathrm{pg}} / \partial V\right)_{T,\left\{x_{i}\right\}}=0$, and

$$
\left(\frac{\partial C_{V}}{\partial V}\right)_{T,\left\{x_{i}\right\}}=T\left(\frac{\partial^{2} P}{\partial T^{2}}\right)_{V,\left\{x_{i}\right\}},
$$

the molar isochoric residual constant-volume heat capacity $C_{V}^{\mathrm{r}}\left(T, V,\left\{x_{i}\right\}\right)$ at constant $T$ and $\left\{x_{i}\right\}$ may be calculated via

$$
C_{V}^{\mathrm{r}}\left(T, V,\left\{x_{i}\right\}\right)=T \int_{\infty}^{V}\left(\frac{\partial^{2} P}{\partial T^{2}}\right)_{V,\left\{x_{i}\right\}} \mathrm{d} V=R T \int_{\infty}^{V}\left[T\left(\frac{\partial^{2} Z}{\partial T^{2}}\right)_{V,\left\{x_{i}\right\}}+2\left(\frac{\partial Z}{\partial T}\right)_{V,\left\{x_{i}\right\}}\right] \frac{\mathrm{d} V}{V} .
$$

Clearly,

$$
\left.\begin{array}{l}
H^{\mathrm{r}}=U^{\mathrm{r}}+P^{\mathrm{r}} V=U^{\mathrm{r}}+R T(Z-1), \\
F^{\mathrm{r}}=U^{\mathrm{r}}-T S^{\mathrm{r}}=-R T \int_{\infty}^{V}(Z-1) \frac{\mathrm{d} V}{V}, \\
G^{\mathrm{r}}=H^{\mathrm{r}}-T S^{\mathrm{r}}=F^{\mathrm{r}}+R T(Z-1),
\end{array}\right\}
$$

and all three functions approach zero for $V \rightarrow \infty$ (i.e., for $P \rightarrow 0$ ). For the computation of values $M$ of any thermodynamic fluid property,

$$
M\left(T, V,\left\{x_{i}\right\}\right)=M^{\mathrm{r}}\left(T, V,\left\{x_{i}\right\}\right)+M^{\mathrm{pg}}\left(T, V,\left\{x_{i}\right\}\right)
$$

is used.

The two types of residual functions, i.e., $M^{\mathrm{R}}\left(T, P,\left\{x_{i}\right\}\right)$ and $M^{\mathrm{r}}\left(T, V,\left\{x_{i}\right\}\right)$, are rigorously related:

$$
M^{\mathrm{r}}\left(T, V,\left\{x_{i}\right\}\right)=M^{\mathrm{R}}\left(T, P,\left\{x_{i}\right\}\right)+\int_{R T / V}^{P}\left(\frac{\partial M^{\mathrm{pg}}}{\partial P}\right)_{T,\left\{x_{i}\right\}} \mathrm{d} P .
$$

Note that $P$ and $V$ are parameters associated with the state of the real fluid system at temperature $T$ and constant composition $\left\{x_{i}\right\}$, and are therefore not related by the perfectgas law: the lower integral limit denotes the gas-pressure $P=R T / V$ for which the molar volume of the perfect-gas mixture has the same value $V$ as that of the real mixture at $T$ and $\left\{x_{i}\right\}$.

Alternatively, we have

$$
M^{\mathrm{R}}\left(T, P,\left\{x_{i}\right\}\right)=M^{\mathrm{r}}\left(T, V,\left\{x_{i}\right\}\right)+\int_{R T / P}^{V}\left(\frac{\partial M^{\mathrm{pg}}}{\partial V}\right)_{T,\left\{x_{i}\right\}} \mathrm{d} V .
$$

Note that $V^{\mathrm{r}}\left(T, V,\left\{x_{i}\right\}\right)$ and $P^{\mathrm{R}}\left(T, P,\left\{x_{i}\right\}\right)$ are identically zero.

Since at constant composition the perfect-gas properties $U^{\mathrm{pg}}, H^{\mathrm{pg}}, C_{P}^{\mathrm{pg}}$ and $C_{V}^{\mathrm{pg}}$ are all functions of temperature only, i.e., the first-law properties are independent of pressure and of volume, the equality 


$$
M^{\mathrm{R}}\left(T, P,\left\{x_{i}\right\}\right)=M^{\mathrm{r}}\left(T, V,\left\{x_{i}\right\}\right) \text { holds for } M=U, H, C_{P} \text { and } C_{V} .
$$

In contradistinction, the second-law perfect-gas properties $S^{\mathrm{pg}}, G^{\mathrm{pg}}$ and $F^{\mathrm{pg}}$ are functions of temperature and they do depend on pressure:

$$
\begin{gathered}
\left(\frac{\partial S^{\mathrm{pg}}}{\partial P}\right)_{T,\left\{x_{i}\right\}}=-\frac{R}{P}, \\
\left(\frac{\partial G^{\mathrm{pg}}}{\partial P}\right)_{T,\left\{x_{i}\right\}}=\left(\frac{\partial F^{\mathrm{pg}}}{\partial P}\right)_{T,\left\{x_{i}\right\}}=\frac{R T}{P},
\end{gathered}
$$

and they do depend on volume:

$$
\begin{gathered}
\left(\frac{\partial S^{\mathrm{pg}}}{\partial V}\right)_{T,\left\{x_{i}\right\}}=\frac{R}{V}, \\
\left(\frac{\partial F^{\mathrm{pg}}}{\partial V}\right)_{T,\left\{x_{i}\right\}}=\left(\frac{\partial G^{\mathrm{pg}}}{\partial V}\right)_{T,\left\{x_{i}\right\}}=-\frac{R T}{V} .
\end{gathered}
$$

Hence, by virtue of Eq. 110 or Eq. 111, respectively, the following relations between the residual second-law properties are obtained:

$$
\begin{gathered}
S^{\mathrm{r}}\left(T, V,\left\{x_{i}\right\}\right)=S^{\mathrm{R}}\left(T, P,\left\{x_{i}\right\}\right)-R \ln Z, \\
F^{\mathrm{r}}\left(T, V,\left\{x_{i}\right\}\right)=F^{\mathrm{R}}\left(T, P,\left\{x_{i}\right\}\right)+R T \ln Z, \\
G^{\mathrm{r}}\left(T, V,\left\{x_{i}\right\}\right)=G^{\mathrm{R}}\left(T, P,\left\{x_{i}\right\}\right)+R T \ln Z .
\end{gathered}
$$

\subsection{Isobaric Residual Properties, Fugacities and Fugacity Coefficients}

One of the most important functions in solution chemistry is the fugacity coefficient $\phi_{i}^{\pi}\left(T, P,\left\{x_{i}^{\pi}\right\}\right)$ of component $i$ in solution in phase $\pi$ [66]. It is related to the isobaric residual chemical potential, which may be obtained either by applying the partial molar derivative prescription

$$
M_{i}\left(T, P,\left(x_{i}\right)\right)=\left[\frac{\partial(n M)}{\partial n_{i}}\right]_{T, P, n_{j \neq i}},
$$

to the expression for the molar isobaric residual Gibbs energy of the mixture in phase $\pi$,

$$
\begin{aligned}
G^{\mathrm{R}, \pi}\left(T, P,\left\{x_{i}^{\pi}\right\}\right) & =G^{\pi}\left(T, P,\left\{x_{i}^{\pi}\right\}\right) \\
& -\sum_{i}^{c} x_{i}^{\pi} G_{i}^{\mathrm{pg}, *}(T, P)-R T \sum_{i}^{c} x_{i}^{\pi} \ln x_{i}^{\pi}
\end{aligned}
$$

or by substituting the expression for the chemical potential of component $i$ in a perfect-gas mixture 


$$
\begin{aligned}
\mu_{i}^{\mathrm{pg}}\left(T, P,\left\{x_{i}^{\pi}\right\}\right) & =G_{i}^{\mathrm{pg}}\left(T, P,\left\{x_{i}^{\pi}\right\}\right) \\
& =G_{i}^{\mathrm{pg}, *}(T, P)+R T \ln x_{i}^{\pi}
\end{aligned}
$$

directly into the defining equation for the isobaric residual chemical potential (the two formulations follow from Eq. 52b). Hence the partial molar isobaric residual Gibbs energy/the isobaric residual chemical potential of component $i$ in solution in phase $\pi$ reads

$$
\begin{aligned}
G_{i}^{\mathrm{R}, \pi}\left(T, P,\left\{x_{i}^{\pi}\right\}\right) & =\mu_{i}^{\mathrm{R}, \pi}\left(T, P,\left\{x_{i}^{\pi}\right\}\right) \equiv G_{i}^{\pi}\left(T, P,\left\{x_{i}^{\pi}\right\}\right) \\
& \left.-G_{i}^{\mathrm{pg}, *}(T, P)-R T \ln x_{i}^{\pi}=\mu_{i}^{\pi}\left(T, P,\left\{x_{i}^{\pi}\right\}\right)-G_{i}^{\mathrm{pg}, *}(T, P)-R T \ln x_{i}^{\pi}\right\},
\end{aligned}
$$

providing a rigorous basis for the definition of the fugacity coefficient of component $i$ in solution in phase $\pi$. The fugacity concept was introduced by Lewis [67]. It serves to maintain the simple formal structure of thermodynamic equations applicable to perfectgas (ideal-gas) systems, while avoiding the troublesome behavior of the chemical potential when either $P$ or $x_{i}^{\pi}$ approaches zero. Thus, in analogy to the expression for an isothermal change of the chemical potential of component $i$ in a perfect-gas mixture,

$$
\mathrm{d} G_{i}^{\mathrm{pg}}=\mathrm{d} \mu_{i}^{\mathrm{pg}}=R T \mathrm{~d} \ln \left(x_{i} P\right)=R T \mathrm{~d} \ln P_{i}, \text { constant } T,
$$

where $P_{i}=x_{i} P$ is the partial pressure of component $i$, the fugacity $f_{i}^{\pi}\left(T, P,\left\{x_{i}^{\pi}\right\}\right)$ of component $i$ in a real solution phase $\pi$ with composition $\left\{x_{i}^{\pi}\right\}$ is partially defined by.

$$
\mathrm{d} G_{i}^{\pi}=\mathrm{d} \mu_{i}^{\pi} \equiv R T \mathrm{~d} \ln f_{i}^{\pi}, \text { constant } T,
$$

For thermodynamic consistency we require as the second part of the definition

$$
f_{i}^{\mathrm{pg}, \pi} \equiv x_{i}^{\pi} P
$$

that is,

$$
\lim _{P \rightarrow 0}\left(\frac{f_{i}^{\pi}}{x_{i}^{\pi} P}\right) \equiv 1, \text { constant } T .
$$

Equations 125 and 126 together constitute the complete definition of the fugacity $f_{i}^{\pi}$ of component $i$ in solution in phase $\pi$. General integration of Eq. 125 at constant temperature [17] from the state of component $i$ in a perfect-gas mixture to the state of $i$ in the real mixture (phase $\pi$ ) at the same pressure and the same composition yields

$$
\begin{aligned}
G_{i}^{\mathrm{R}, \pi}\left(T, P,\left\{x_{i}^{\pi}\right\}\right) & \equiv \mu_{i}^{\mathrm{R}, \pi}\left(T, P,\left\{x_{i}^{\pi}\right\}\right) \\
\equiv & G_{i}^{\pi}\left(T, P,\left\{x_{i}^{\pi}\right\}\right)-G_{i}^{\mathrm{pg}}\left(T, P,\left\{x_{i}^{\pi}\right\}\right)=R T \ln \frac{f_{i}^{\pi}\left(T, P,\left\{x_{i}^{\pi}\right\}\right)}{x_{i}^{\pi} P},
\end{aligned}
$$

where the partial molar residual Gibbs energy in $(T, P, x)$-space of component $i$ in solution in phase $\pi, G_{i}^{\mathrm{R}, \pi}\left(T, P,\left\{x_{i}^{\pi}\right\}\right)$, and the residual chemical potential in $(T, P, x)$-space of component $i$ in solution in phase $\pi, \mu_{i}^{\mathrm{R}, \pi}\left(T, P,\left\{x_{i}^{\pi}\right\}\right)$, are given by Eq. 123. The dimensionless ratio appearing on the right-hand side of Eq. 128 as the argument of the logarithm is a new property and is called the fugacity coefficient $\phi_{i}^{\pi}$ of component $i$ in solution in phase $\pi$ : 


$$
\left.\phi_{i}^{\pi}\left(T, P,\left\{x_{i}^{\pi}\right\}\right) \equiv \frac{f_{i}^{\pi}\left(T, P,\left\{x_{i}^{\pi}\right\}\right)}{x_{i}^{\pi} P}=\exp \left(\frac{G_{i}^{\mathrm{R}, \pi}\left(T, P,\left\{x_{i}^{\pi}\right\}\right)}{R T}\right)\right\}
$$

or, perhaps, more convenient,

$$
\ln \left[\phi_{i}^{\pi}\left(T, P,\left\{x_{i}^{\pi}\right\}\right)\right]=\frac{G_{i}^{\mathrm{R}, \pi}\left(T, P,\left\{x_{i}^{\pi}\right\}\right)}{R T}=\frac{\mu_{i}^{\mathrm{R}, \pi}\left(T, P,\left\{x_{i}^{\pi}\right\}\right)}{R T} .
$$

For a perfect-gas system, necessarily $G_{i}^{\mathrm{R}, \pi}=\mu_{i}^{\mathrm{R}, \pi}=0$ and thus $\phi_{i}^{\mathrm{pg}}=1$. For a real pure substance $i$ we obtain

$$
G_{i}^{\mathrm{R}, \pi, *}(T, P) \equiv G_{i}^{\pi, *}(T, P)-G_{i}^{\mathrm{pg}, *}(T, P)=R T \ln \frac{f_{i}^{\pi, *}(T, P)}{P},
$$

where $G_{i}^{\mathrm{R}, \pi, *}(T, P)$ denotes the molar isobaric residual Gibbs energy of pure component $i$ in phase $\pi, f_{i}^{\pi, *}(T, P)$ is its fugacity, and

$$
\phi_{i}^{\pi, *}(T, P) \equiv \frac{f_{i}^{\pi, *}(T, P)}{P}=\exp \left(\frac{G_{i}^{\mathrm{R}, \pi, *}(T, P)}{R T}\right)
$$

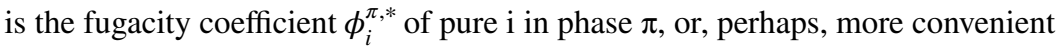

$$
\ln \left[\phi_{i}^{\pi, *}(T, P)\right]=\frac{G_{i}^{\mathrm{R}, \pi, *}(T, P)}{R T}
$$

For the overall fugacity of the solution in phase $\pi$ we have

$$
G^{\mathrm{R}, \pi}\left(T, P,\left\{x_{i}^{\pi}\right\}\right) \equiv G^{\pi}\left(T, P,\left\{x_{i}^{\pi}\right\}\right)-G^{\mathrm{pg}}\left(T, P,\left\{x_{i}^{\pi}\right\}\right)=R T \ln \frac{f^{\pi}\left(T, P,\left\{x_{i}^{\pi}\right\}\right)}{P},
$$

where $G^{\mathrm{R}, \pi}\left(T, P,\left\{x_{i}^{\pi}\right\}\right)$ is the molar isobaric residual Gibbs energy of the solution, and

$$
\phi^{\pi}\left(T, P,\left\{x_{i}^{\pi}\right\}\right) \equiv \frac{f^{\pi}\left(T, P,\left\{x_{i}^{\pi}\right\}\right)}{P}=\exp \left(\frac{G^{\mathrm{R}, \pi}\left(T, P,\left\{x_{i}^{\pi}\right\}\right)}{R T}\right),
$$

is the overall fugacity coefficient of the solution in phase $\pi$, or, perhaps, more convenient,

$$
\ln \left[\phi^{\pi}\left(T, P,\left\{x_{i}^{\pi}\right\}\right)\right]=\frac{G^{\mathrm{R}, \pi}\left(T, P,\left\{x_{i}^{\pi}\right\}\right)}{R T} .
$$

Evidently, fugacity, having the dimension of pressure, and the dimensionless fugacity coefficient are intensive state functions related to exponentials of (partial) molar isobaric residual Gibbs energies (divided by $R T$ ) as indicated above by Eqs. 129, 132 and 135. Viewing the fugacity as a "corrected pressure" may be misleading and obscure these exact relations. As the pressure goes to zero (at constant temperature and composition), the (partial) molar residual Gibbs energies divided by $R T$, as introduced above, all approach zero on the basis that the perfect-gas state is approached asymptotically; hence $f_{i}^{\pi} \rightarrow x_{i}^{\pi} P \equiv P_{i}$, $f_{i}^{\pi, *} \rightarrow P$ and $f^{\pi} \rightarrow P$, respectively. Thus, 


$$
\begin{gathered}
\lim _{P \rightarrow 0} \frac{f_{i}^{\pi}}{x_{i}^{\pi} P}=\lim _{P \rightarrow 0} \phi_{i}^{\pi}=1, \\
\lim _{P \rightarrow 0} \frac{f_{i}^{\pi, *}}{P}=\lim _{P \rightarrow 0} \phi_{i}^{\pi, *}=1,
\end{gathered}
$$

and

$$
\lim _{P \rightarrow 0} \frac{f^{\pi}}{P}=\lim _{P \rightarrow 0} \phi^{\pi}=1
$$

The fugacity coefficients are always positive: for real systems, they may be larger than 1 or smaller than 1 , and they are unity for a perfect-gas system.

Since $G_{i}^{\mathrm{R}, \pi}\left(T, P,\left\{x_{i}^{\pi}\right\}\right)$ is the partial molar isobaric residual Gibbs energy of component $i$ in phase $\pi$, i.e.,

$$
G_{i}^{\mathrm{R}, \pi}\left(T, P,\left\{x_{i}^{\pi}\right\}\right) \equiv\left(\frac{\partial\left(n^{\pi} G^{\mathrm{R}, \pi}\right)}{\partial n_{i}^{\pi}}\right)_{T, P, n_{j \neq i}^{\pi}},
$$

the associated summability relation yields the molar residual Gibbs energy of the solution

$$
G^{\mathrm{R}, \pi}\left(T, P,\left\{x_{i}^{\pi}\right\}\right)=\sum_{i}^{c} x_{i}^{\pi} G_{i}^{\mathrm{R}, \pi}\left(T, P,\left\{x_{i}^{\pi}\right\}\right) .
$$

For convenience, the non-dimensional quantity $G^{\mathrm{R}, \pi} / R T$ is frequently used instead of $G^{\mathrm{R}, \pi}$, hence

$$
\begin{aligned}
\frac{G^{\mathrm{R}, \pi}\left(T, P,\left\{x_{i}^{\pi}\right\}\right)}{R T} & =\sum_{i}^{c} x_{i}^{\pi} \frac{G_{i}^{\mathrm{R}, \pi}\left(T, P,\left\{x_{i}^{\pi}\right\}\right)}{R T} \\
& \left.=\sum_{i}^{c} x_{i}^{\pi} \frac{\mu_{i}^{\mathrm{R}, \pi}\left(T, P,\left\{x_{i}^{\pi}\right\}\right)}{R T}=\sum_{i}^{c} x_{i}^{\pi} \ln \phi_{i}^{\pi}\left(T, P,\left\{x_{i}^{\pi}\right\}\right)=\ln \phi^{\pi}\left(T, P,\left\{x_{i}^{\pi}\right\}\right) .\right\}
\end{aligned}
$$

We recognize that $\ln \phi_{i}^{\pi}$ is a partial molar property in relation to $\ln \phi^{\pi}$, where $\phi^{\pi}$ is the overall fugacity coefficient of the solution in phase $\pi$ :

$$
\begin{aligned}
\ln \phi_{i}^{\pi}\left(T, P,\left\{x_{i}^{\pi}\right\}\right) & =\left(\frac{\partial\left[n^{\pi} \ln \phi^{\pi}\left(T, P,\left\{x_{i}^{\pi}\right\}\right)\right]}{\partial n_{i}^{\pi}}\right)_{T, P, n_{j \neq i}^{\pi}}=\left(\frac{\partial\left[n^{\pi} G^{\mathrm{R}, \pi}\left(T, P,\left\{x_{i}^{\pi}\right\}\right) / R T\right]}{\partial n_{i}^{\pi}}\right)_{T, P, n_{j \neq i}^{\pi}} \\
& \left.=\frac{G_{i}^{\mathrm{R}, \pi}\left(T, P,\left\{x_{i}^{\pi}\right\}\right)}{R T}=\frac{\mu_{i}^{\mathrm{R}, \pi}\left(T, P,\left\{x_{i}^{\pi}\right\}\right)}{R T} \cdot\right\}
\end{aligned}
$$

The fundamental residual-property relation (canonical variables $T, P, n_{i}^{\pi}$ ) valid for a fluid in any phase $\pi$, may now be written as 


$$
\begin{aligned}
\mathrm{d}\left(\frac{n^{\pi} G^{\mathrm{R}, \pi}}{R T}\right)= & -\frac{n^{\pi} H^{\mathrm{R}, \pi}}{R T^{2}} \mathrm{~d} T \\
& +\frac{n^{\pi} V^{\mathrm{R}, \pi}}{R T} \mathrm{~d} P+\sum_{i}^{c} \frac{\mu_{i}^{\mathrm{R}, \pi}}{R T} \mathrm{~d} n_{i}^{\pi},
\end{aligned}
$$

or, alternatively,

$$
\begin{aligned}
\mathrm{d}\left(n^{\pi} \ln \phi^{\pi}\right)= & -\frac{n^{\pi} H^{\mathrm{R}, \pi}}{R T^{2}} \mathrm{~d} T \\
& +\frac{n^{\pi} V^{\mathrm{R}, \pi}}{R T} \mathrm{~d} P+\sum_{i}^{c}\left(\ln \phi_{i}^{\pi}\right) \mathrm{d} n_{i}^{\pi} .
\end{aligned}
$$

Here,

$$
\left.-\frac{H^{\mathrm{R}, \pi}\left(T, P,\left\{x_{i}^{\pi}\right\}\right)}{R T^{2}}=\left(\frac{\partial\left(G^{\mathrm{R}, \pi} / R T\right)}{\partial T}\right)_{P,\left\{x_{i}^{\pi}\right\}}=\left(\frac{\partial \ln \phi^{\pi}}{\partial T}\right)_{P,\left\{x_{i}^{\pi}\right\}}\right\},
$$

where $H^{\mathrm{R}, \pi}$ is the molar isobaric residual enthalpy of the solution in phase $\pi$, that is,

$$
H^{\mathrm{R}, \pi}\left(T, P,\left\{x_{i}^{\pi}\right\}\right)=H^{\pi}\left(T, P,\left\{x_{i}^{\pi}\right\}\right)-H^{\mathrm{pg}}\left(T, P,\left\{x_{i}^{\pi}\right\}\right) .
$$

and

$$
\left.\frac{V^{\mathrm{R}, \pi}\left(T, P,\left\{x_{i}^{\pi}\right\}\right)}{R T}=\left(\frac{\partial\left(G^{\mathrm{R}, \pi} / R T\right)}{\partial P}\right)_{T,\left\{x_{i}^{\pi}\right\}}=\left(\frac{\partial \ln \phi^{\pi}}{\partial P}\right)_{T,\left\{x_{i}^{\pi}\right\}}\right\} .
$$

where $V^{\mathrm{R}, \pi}$ is the molar isobaric residual volume of the solution in phase $\pi$, that is,

$$
V^{\mathrm{R}, \pi}\left(T, P,\left\{x_{i}^{\pi}\right\}\right)=V^{\pi}\left(T, P,\left\{x_{i}^{\pi}\right\}\right)-V^{\mathrm{pg}}\left(T, P,\left\{x_{i}^{\pi}\right\}\right) .
$$

Evidently, the partial molar property analogues of Eqs. 145 and 147 are

$$
\left(\frac{\partial \ln \phi_{i}^{\pi}}{\partial T}\right)_{P,\left\{x_{i}^{\pi}\right\}}=-\frac{H_{i}^{\mathrm{R}, \pi}}{R T^{2}}=-\frac{H_{i}^{\pi}-H_{i}^{\mathrm{pg}, *}}{R T^{2}},
$$

and

$$
\left(\frac{\partial \ln \phi_{i}^{\pi}}{\partial P}\right)_{T,\left\{x_{i}^{\pi}\right\}}=\frac{V_{i}^{\mathrm{R}, \pi}}{R T}=\frac{V_{i}^{\pi}-V_{i}^{\mathrm{pg}, *}}{R T},
$$

respectively. Here, $H_{i}^{\mathrm{R}, \pi}$ is the partial molar isobaric residual enthalpy, and $V_{i}^{\mathrm{R}, \pi}$ is the partial molar isobaric residual volume of component $i$ in solution in phase $\pi$, respectively. The corresponding summability relations read 


$$
H^{\mathrm{R}, \pi}\left(T, P,\left\{x_{i}^{\pi}\right\}\right)=\sum_{i}^{c} x_{i}^{\pi} H_{i}^{\mathrm{R}, \pi}\left(T, P,\left\{x_{i}^{\pi}\right\}\right)
$$

and

$$
V^{\mathrm{R}, \pi}\left(T, P,\left\{x_{i}^{\pi}\right\}\right)=\sum_{i}^{c} x_{i}^{\pi} V_{i}^{\mathrm{R}, \pi}\left(T, P,\left\{x_{i}^{\pi}\right\}\right) .
$$

Evaluation of $\ln \phi_{i}^{\pi}\left(T, P,\left\{x_{i}^{\pi}\right\}\right)$, using an equation of state in conjunction with Eq. 150, is now straightforward. Since the molar volume of pure component $i$ in the perfect-gas state is given by $V_{i}^{\mathrm{pg}, *}=R T / P$, and its partial molar volume in phase $\pi$ by $V_{i}^{\pi}=\frac{R T}{P}\left[\partial\left(n^{\pi} Z^{\pi}\right) / \partial n_{i}^{\pi}\right]_{T, P,\left\{n_{j \neq i}^{\pi}\right\}}$, integration at constant $T$ and constant composition from $P=0\left(\right.$ where $\left.\phi_{i}^{\pi}=1\right)$ to arbitrary pressure $P$ yields

$$
\ln \phi_{i}^{\pi}\left(T, P,\left\{x_{i}^{\pi}\right\}\right)=\int_{0}^{P}\left[\left(\frac{\partial\left(n^{\pi} Z^{\pi}\right)}{\partial n_{i}^{\pi}}\right)_{T, P,\left\{n_{j \neq i}^{\pi}\right\}}-1\right] \frac{\mathrm{d} P}{P}, \text { constant } T,\left\{x_{i}\right\},
$$

Equation 153a is a generally valid relation for the determination of $\ln \phi_{i}^{\pi}\left(T, P,\left\{x_{i}^{\pi}\right\}\right)$ from any volume-explicit EOS. By way of example, consider the binary vapor-phase mixture $\left(\pi=\mathrm{V}, x_{1}^{\mathrm{V}}=1-x_{2}^{\mathrm{V}}\right)$ adequately described by a two-term virial equation in pressure, i.e., $Z^{\mathrm{V}}\left(T, P, x_{2}^{\mathrm{V}}\right)=1+\frac{B\left(T, x_{2}\right)}{R T} P$. After some mathematical manipulation we obtain the compact expression for the fugacity coefficients of component 1 and 2, respectively, in the binary vapor mixture:

$$
\ln \phi_{i}^{\mathrm{V}}\left(T, P, x_{i}^{\mathrm{V}}\right)=\frac{P}{R T}\left(B_{i i}(T)+\left(x_{j}^{\mathrm{V}}\right)^{2} \delta_{12}(T)\right), i, j=1,2, i \neq j .
$$

Here, $B\left(T, x_{2}^{\mathrm{V}}\right)=x_{1}^{\mathrm{V}} B_{11}(T)+x_{2}^{\mathrm{V}} B_{22}(T)+x_{1}^{\mathrm{V}} x_{2}^{\mathrm{V}} \delta_{12}(T)$ is the second virial coefficient of the mixture, $\delta_{12} \equiv 2 B_{12}-\left(B_{11}+B_{22}\right), B_{11}$ and $B_{22}$ are the second virial coefficients of the pure components, and $B_{12}$ designates a composition-independent interaction virial coefficient (cross-coefficient). When focusing on highly dilute systems, the fugacity coefficient of, say, component 2 at infinite dilution in the vapor phase is thus given by

$$
\ln \phi_{2}^{\mathrm{V}, \infty}(T, P)=\frac{P}{R T}\left(2 B_{12}-B_{11}\right),
$$

For pure substance $i$ in phase $\pi$ we have

$$
\left(\frac{\partial \ln \phi_{i}^{\pi, *}}{\partial T}\right)_{P}=-\frac{H_{i}^{\mathrm{R}, \pi, *}}{R T^{2}}=-\frac{H_{i}^{\pi, *}-H_{i}^{\mathrm{pg}, *}}{R T^{2}},
$$

and

$$
\left(\frac{\partial \ln \phi_{i}^{\pi, *}}{\partial P}\right)_{T}=\frac{V_{i}^{\mathrm{R}, \pi, *}}{R T}=\frac{V_{i}^{\pi, *}-V_{i}^{\mathrm{pg}, *}}{R T} .
$$

To conclude this section, I emphasize that $G^{\mathrm{R}, \pi}\left(T, P,\left\{x_{i}^{\pi}\right\}\right) / R T$ is a convenient generating function for molar isobaric residual properties (see Eq. 144a). 
In principle, the exact classical thermodynamic method of using isobaric residual functions for the calculation of property changes of single-phase constant-composition fluids for any arbitrary equilibrium change of state is a quite general and powerful tool; and applications profit greatly from contributions based on the corresponding states theorem, such as the popular Lee-Kesler tables [56-58]. However, for a complete description, the perfectgas heat capacities $C_{P}^{\mathrm{pg}}\left(T,\left\{x_{i}\right\}\right)$ must be available.

To illustrate the convenient use of isobaric residual functions in the calculation of property changes for single-phase, constant-composition fluids (pure or multicomponent systems), consider an arbitrary change of state, say, from an initial condition characterized by $\left\{T_{1}, P_{1}\right\}$ to a final condition $\left\{T_{2}, P_{2}\right\}$. Such a change of state causes a change

$$
\Delta_{1 \rightarrow 2} M=M\left(T_{2}, P_{2},\left\{x_{i}\right\}\right)-M\left(T_{1}, P_{1},\left\{x_{i}\right\}\right),
$$

of any molar thermodynamic property $M\left(T, P,\left\{x_{i}\right\}\right)$. When interest focuses on $M=H$, the change of enthalpy per mole of mixture $\Delta_{1 \rightarrow 2} H$ is given by

$$
\begin{aligned}
\Delta_{1 \rightarrow 2} H= & H_{2}^{\mathrm{R}}\left(T_{2}, P_{2},\left\{x_{i}\right\}\right) \\
& -H_{1}^{\mathrm{R}}\left(T_{1}, P_{1},\left\{x_{i}\right\}\right)+\int_{T_{1}}^{T_{2}} C_{P}^{\mathrm{pg}}\left(T,\left\{x_{i}\right\}\right) \mathrm{d} T,
\end{aligned}
$$

Here, $\left(\partial H^{\mathrm{pg}} / \partial P\right)_{T,\left\{x_{i}\right\}}=0$ and $\left(\partial H^{\mathrm{pg}} / \partial T\right)_{P,\left\{x_{i}\right\}}=C_{P}^{\mathrm{pg}}$ were taken into account. For $M=S$, the change of entropy per mole of mixture $\Delta_{1 \rightarrow 2} S$ is given by

$$
\begin{aligned}
\Delta_{1 \rightarrow 2} S= & S_{2}^{\mathrm{R}}\left(T_{2}, P_{2},\left\{x_{i}\right\}\right)-S_{1}^{\mathrm{R}}\left(T_{1}, P_{1},\left\{x_{i}\right\}\right) \\
& +\int_{T_{1}}^{T_{2}}\left[C_{P}^{\mathrm{pg}}\left(T,\left\{x_{i}\right\}\right) / T\right] \mathrm{d} T-R \ln \left(P_{2} / P_{1}\right),
\end{aligned}
$$

since $\mathrm{d} S^{\mathrm{pg}}=\left(C_{P}^{\mathrm{pg}} / T\right) \mathrm{d} T-(R / P) \mathrm{d} P$. Evaluation of the integrals involving $C_{P}^{\mathrm{pg}}$ requires empirical expressions for the temperature dependence of the perfect-gas heat capacities involved [68]. For pure fluids, a commonly used empirical equation [49] reads

$$
\frac{C_{P}^{\mathrm{pg}, *}(T)}{R}=C_{0}+C_{1} T+C_{2} T^{2}+C_{3} T^{3}+C_{4} T^{4},
$$

and extensive data compilations are available [69-71]. For fairly simple molecules, $C_{P}^{\mathrm{pg}, *}(T)$ may be calculated using the frequencies of their normal modes of vibration [72-74].

The heat capacities of perfect-gas mixtures are rigorously obtained by mole-fraction averaging the pure-substance perfect-gas heat capacities, that is,

$$
\left.\begin{array}{l}
C_{P}^{\mathrm{pg}}\left(T,\left\{x_{i}\right\}\right)=\sum_{i}^{c} x_{i} C_{P i}^{\mathrm{pg}, *}(T), \\
C_{V}^{\mathrm{pg}}\left(T,\left\{x_{i}\right\}\right)=\sum_{i}^{c} x_{i} C_{V i}^{\mathrm{pg}, *}(T),
\end{array}\right\},
$$


and their difference is the same as that for pure perfect gases, $C_{P}^{\mathrm{pg}}\left(T,\left\{x_{i}\right\}\right)-C_{V}^{\mathrm{pg}}\left(T,\left\{x_{i}\right\}\right)=R$.

Since the perfect-gas state is characterized by the absence of intermolecular forces, the corresponding-states theorem is inapplicable, and when experimental data on $C_{P}^{\mathrm{pg}, *}(T)$ are lacking, reliable estimation methods based on molecular structure have to be used [49, 75-77].

\subsection{Empirical Equations of State: Selected Comments}

From $P V T$ equations of state, volumetric properties as well as residual functions characterizing deviations from perfect-gas (ideal-gas) behavior can be calculated (see Sects. 2.1 and 2.2). The PVT relation may be a pressure-explicit EOS or a volume-explicit (amount density-explicit) EOS. The simplest, practically useful polynomial EOS are cubic in molar volume (i.e., they are pressure-explicit), since they are capable of yielding the perfect-gas limit for $V \rightarrow \infty$, and of representing both liquid-like and vapor-like volumes for sufficiently low temperatures. The five-parameter equation [17]

$$
P=\frac{R T}{V-b}-\frac{\theta(V-\eta)}{(V-b)\left(V^{2}+\delta V+\varepsilon\right)},
$$

where the adjustable parameters $b, \theta, \eta, \delta, \varepsilon$ depend, in general, on temperature and composition, can be considered a generalization of the vdW equation Eq. 3 [61], to which it reduces for $\eta=b, \delta=\varepsilon=0$, and $\theta=$ constant $=a$. Over the decades, many specializations of Eq. 161 have been suggested [78], two of the most popular being the Redlich-Kwong (RK) Eq. (1949) [79],

$$
P=\frac{R T}{V-b_{\mathrm{RK}}}-\frac{a_{\mathrm{RK}}}{T^{1 / 2} V\left(V+b_{\mathrm{RK}}\right)},
$$

where $b=b_{\mathrm{RK}}, \theta=\theta_{\mathrm{RK}}(T) \equiv a_{\mathrm{RK}} / T^{1 / 2}, \eta=b_{\mathrm{RK}}, \delta=b_{\mathrm{RK}}$ and $\varepsilon=0$, and the Peng-Robinson (PR) Eq. (1976) [80],

$$
P=\frac{R T}{V-b_{\mathrm{PR}}}-\frac{\theta_{\mathrm{PR}}(T)}{V^{2}+2 b_{\mathrm{PR}} V-b_{\mathrm{PR}}^{2}},
$$

where $b=b_{\mathrm{PR}}, \theta=\theta_{\mathrm{PR}}(T) \equiv a_{\mathrm{PR}}(T), \eta=b_{\mathrm{PR}}, \delta=2 b_{\mathrm{PR}}$, and $\varepsilon=-b_{\mathrm{PR}}^{2}$.

Significantly, all modern cubic equations of state have a temperature-dependent $\theta$. While the RK equation gives a somewhat better critical compression factor than the original van der Waals EOS (see Eq. 8 and associated comments), i.e.,

$$
Z_{\mathrm{c}, \mathrm{RK}}=\frac{1}{3},
$$

and better second virial coefficients [81], it is still not very accurate for vapor pressures and liquid densities. It was Soave (S) [82, 83] who generalized, in 1972, the temperature dependence of the attractive parameter in the RK equation by writing, with $\left.\theta=\theta_{\mathrm{S}}(T) \equiv a_{\mathrm{S}}(T)\right)$

$$
P=\frac{R T}{V-b_{\mathrm{S}}}-\frac{a_{\mathrm{S}}(T)}{V\left(V+b_{\mathrm{S}}\right)} .
$$


In order to obtain the EOS parameters $a_{\mathrm{S}}(T)$ and $b_{\mathrm{S}}$, the most frequently employed conventional approach makes use of the mathematical requirements for the occurrence of an inflection point on the critical isotherm in the $\{P, V\}$-plane, that is, $(\partial P / \partial V)_{T_{\mathrm{c}}}=0$ and $\left(\partial^{2} P / \partial V^{2}\right)_{T_{\mathrm{c}}}=0$ at the critical point $\left(P=P_{\mathrm{c}}, V=V_{\mathrm{c}}\right)$. When applying these classical critical constraints, experience shows that the preferred sets of expressions for evaluating $a_{\mathrm{S}}\left(T_{\mathrm{c}}\right) \equiv a_{\mathrm{S}, \mathrm{c}}$ and $b_{\mathrm{S}}$ are in terms of $T_{\mathrm{c}}$ and $P_{\mathrm{c}}$, simply because critical pressures are known for more substances with better precision than critical molar volumes $V_{\mathrm{c}}[1,49]$. For the Soave equation of state we obtain

$$
\theta_{\mathrm{S}}\left(T_{\mathrm{c}}\right) \equiv a_{\mathrm{S}}\left(T_{\mathrm{c}}\right) \equiv a_{\mathrm{S}, \mathrm{c}}=0.42748 \frac{R^{2} T_{\mathrm{c}}^{2}}{P_{\mathrm{c}}} \text { and } b_{\mathrm{S}}=0.08664 \frac{R T_{\mathrm{c}}}{P_{\mathrm{c}}},
$$

and the same value for the critical compression factor as that of the Redlich-Kwong equation of state, i.e., $Z_{\mathrm{c}, \mathrm{S}}=\frac{1}{3}$.

The Soave equation of state, Eq. 165, may now be written as

$$
P=\frac{R T}{V-b_{\mathrm{S}}}-\frac{a_{\mathrm{S}, \mathrm{c}} \alpha_{\mathrm{S}}(T, \omega)}{V\left(V+b_{\mathrm{S}}\right)} .
$$

Thus, at temperatures $T \leq T_{\mathrm{c}}$, the attraction parameter $a_{\mathrm{S}}(T)$ is now expressed as the product of its value at the critical point and a dimensionless temperature-dependent $\alpha$-function, that is

$$
a_{\mathrm{S}}(T)=a_{\mathrm{S}, \mathrm{c}} \alpha_{\mathrm{S}}(T, \omega) .
$$

Clearly, for the limiting value of the $\alpha$-function as $T \rightarrow T_{\mathrm{c}}\left(\right.$ i.e., $\left.T_{\mathrm{r}} \rightarrow 1\right)$ we require

$$
\lim _{T \rightarrow T_{\mathrm{c}}} \alpha_{\mathrm{S}}(T, \omega)=1
$$

This empirical function was primarily formulated to help better fit vapor pressure data of hydrocarbons, and Soave suggested as a generally useful form

$$
\left.\begin{array}{rl}
\alpha_{\mathrm{S}}(T, \omega) & =\left[1+w_{\mathrm{S}}\left(1-\sqrt{T / T_{\mathrm{c}}}\right)\right]^{2}, \\
w_{\mathrm{S}} & =0.480+1.574 \omega-0.176 \omega^{2}
\end{array}\right\},
$$

whose limiting value for $T \rightarrow T_{\mathrm{c}}$ is one, in accord with Eq. 169. With a substantially expanded property set of vapor pressure data becoming available (a then new compilation from the American Petroleum Institute), the $\alpha_{S}(T, \omega)$-relation was refitted by Graboski and Daubert [84-86] to yield an improved expression with

$$
w_{\mathrm{S}}=0.48508+1.55171 \omega-0.1561 \omega^{2} .
$$

When applying the classical critical constraints to the Peng-Robinson equation of state, Eq. 163, we obtain

$$
\theta_{\mathrm{PR}}\left(T_{\mathrm{c}}\right) \equiv a_{\mathrm{PR}}\left(T_{\mathrm{c}}\right) \equiv a_{\mathrm{PR}, \mathrm{c}}=0.45724 \frac{R^{2} T_{\mathrm{c}}^{2}}{P_{\mathrm{c}}} \text { and } b_{\mathrm{PR}}=0.07780 \frac{R T_{\mathrm{c}}}{P_{\mathrm{c}}},
$$

and the EOS may be rewritten as follows: 


$$
P=\frac{R T}{V-b_{\mathrm{PR}}}-\frac{a_{\mathrm{PR}, \mathrm{c}} \alpha_{\mathrm{PR}}(T, \omega)}{V^{2}+2 b_{\mathrm{PR}} V-b_{P R}^{2}} .
$$

Thus, at temperatures $T \leq T_{\mathrm{c}}$, the attraction parameter $\theta_{\mathrm{PR}}(T)=a_{\mathrm{PR}}(T)$ is now expressed as the product of its value at the critical point and a dimensionless temperaturedependent $\alpha$-function, that is,

$$
a_{\mathrm{PR}}(T)=a_{\mathrm{PR}, \mathrm{c}} \alpha_{\mathrm{PR}}(T, \omega),
$$

again with the constraint

$$
\lim _{T \rightarrow T_{\mathrm{c}}} \alpha_{\mathrm{PR}}(T, \omega)=1 .
$$

Correlating vapor-pressure data with the Peng-Robinson equation of state yielded

$$
\left.\begin{array}{rl}
\alpha_{\mathrm{PR}}(T, \omega) & =\left[1+w_{\mathrm{PR}}\left(1-\sqrt{T / T_{c}}\right)\right]^{2}, \\
w_{\mathrm{PR}} & =0.37464+1.54226 \omega-0.26992 \omega^{2}
\end{array}\right\} .
$$

Over the years, many other $\alpha$-functions of increasing complexity have been proposed. The Soave-type $\alpha$-function is a generalized, polynomial expression which has been honed over decades, thereby making it so popular. It has been recognized that adequately formulated $\alpha$-functions substantially improved predictions of vapor pressure, especially of polar liquids [87]. However, its behavior was established for subcritical conditions only, and theory-based guidelines for developing adequate $\alpha$-function in general, and for supercritical conditions in particular, have been lacking until very recently. Based on a careful thermodynamic/mathematical analysis of the problem, researchers from Nancy, France, that is, Le Guennec, Lasala, Privat and Jaubert, have established the requirements for consistent $\alpha$-functions to be used in cubic equations of state applied at both subcritical and supercritical conditions [88-90].

The method of parameter evaluation for the PR equation of state remains unchanged, and the critical compression factor is $Z_{\mathrm{c}, \mathrm{PR}}=0.307$. This value is nearer the common experimental values (particularly for nonpolar compounds) and explains partially the fact that the PR equation of state predicts liquid densities more accurately than the Soave equation of state.

Despite the inherent empiricism [91], both the Soave and the Peng-Robinson EOS have remained mainstays for calculating thermodynamic properties of fluids and vapor-liquid equilibria: nearly 150 years after van der Waals' dissertation [24], generalized vdW equations are still a hot topic in chemical engineering.

The greatest utility of cubic equations of state is for vapor-liquid phase equilibrium calculations involving mixtures. Extension of vdW-type model equations to multicomponent mixtures rests upon the inherent assumption that the same EOS used for the pure fluid components can be used for the $c$-component mixture, provided adequate prescriptions for the mixture parameters are available. The van der Waals one-fluid approximation provides such recipes for the composition dependence of the mixture interaction energy parameter $a$, say, $a_{\mathrm{S}}$ or $a_{\mathrm{PR}}$, and of the mixture molecular size parameter $b$, say, $b_{\mathrm{S}}$ or $b_{\mathrm{PR}}$. These mixing rules are quadratic in mole fraction: 


$$
a\left(\left\{x_{i}\right\}\right)=\sum_{i=1}^{c} \sum_{j=1}^{c} x_{i} x_{j} a_{i j},
$$

where $x_{i}$ and $x_{j}$ are the mole fractions of components $i$ and $j$, respectively. The attractive $\mathrm{vdW}$ interaction parameters of the pure components are denoted by $a_{i i}$ and $a_{j j}$, while $a_{i j}$ measures the strength of the attractive interaction between unlike molecules. Similarly, the composition dependence of the mixture parameter $b$ (also known as the mixture covolume) is also approximated by a quadratic mixing rule

$$
b\left(\left\{x_{i}\right\}\right)=\sum_{i=1}^{c} \sum_{j=1}^{c} x_{i} x_{j} b_{i j},
$$

where $b_{i i}$ and $b_{j j}$ denote the vdW size parameters (covolumes) of the pure components, and $b_{i j}$ characterizes the repulsive interaction between unlike molecules.

For three-parameter cubic equations of state, such as the Patel-Teja (PT) equation [92],

$$
P=\frac{R T}{V-b_{\mathrm{PT}}}-\frac{\theta_{\mathrm{PT}}(T)}{V^{2}+V\left(b_{\mathrm{PT}}+c_{\mathrm{PT}}\right)-b_{\mathrm{PT}} c_{\mathrm{PT}}},
$$

where the parameters in the generalized $\mathrm{vdW}$ equation, Eq. 161, are given by $b=b_{\mathrm{PT}}$, $\theta=\theta_{\mathrm{PT}}(T) \equiv a_{\mathrm{PT}}(T), \eta=b_{\mathrm{PT}}, \delta=b_{\mathrm{PT}}+c_{\mathrm{PT}}$, and $\varepsilon=-b_{\mathrm{PT}} c_{\mathrm{PT}}$, a similar mixing rule for the third parameter, here for $c_{\mathrm{PT}}$, is usually assumed:

$$
c_{\mathrm{PT}}\left(\left\{x_{i}\right\}\right)=\sum_{i=1}^{c} \sum_{j=1}^{c} x_{i} x_{j} c_{\mathrm{PT} i j} .
$$

I emphasize that these commonly used mixing rules are semiempirical approximations, and alternative recipes could be used and have indeed been suggested. However, to apply Eqs. 177 and 178 (and 180), for unlike interactions $(i \neq j)$ the cross-interaction parameters $a_{i j}$ and $b_{i j}$ (and $c_{i j}$ ) have to be known: evaluation of these quantities in terms of pure-substance parameters is one of the key problems in molecular thermodynamics [1, 41-43, 49, $60,93,94]$. The most common choices for combining rules are the geometric-mean rule for $a_{i j}$ suggested by Galitzine in 1890 [95], and later by Berthelot in 1898 [96, 97], and the arithmetic-mean rule for $b_{i j}$, though in engineering calculations, both are routinely modified on an empirical basis:

$$
\begin{gathered}
a_{i j}=\left(1-k_{i j}\right) \sqrt{a_{i i} a_{j j}}, \\
b_{i j}=\left(1-l_{i j}\right)\left(b_{i i}+b_{j j}\right) / 2 .
\end{gathered}
$$

The parameters $k_{i j}$ and $l_{i j}$ are known as binary interaction parameters. For three-parameter cubic equations of state, $c_{i j}$ is typically approximated by

$$
c_{i j}=\left(1-m_{i j}\right)\left(c_{i i}+c_{j j}\right) / 2,
$$

$m_{i j}$ being another empirical binary interaction parameter.

So far, every cubic EOS that has been proposed has some limitations, either with respect to the range of operating conditions or types of fluids it could be applied to. Further progress in developing pressure-explicit multiparameter equations of state, was initially 
greatly stimulated by work on the virial equation in the amount density $\rho_{n}=1 / V$, which for a pure gas/vapor at not too high densities reads

$$
\frac{P}{\rho_{n} R T}=Z\left(T, \rho_{n}\right)=1+B(T) \rho_{n}+C(T) \rho_{n}^{2}+\cdots .
$$

This equation has a sound theoretical foundation [98]. Here, $B(T)$ is the second virial coefficient, $C(T)$ is the third virial coefficient, and so forth. The celebrated EOS of Benedict, Webb and Rubin (BWR) [99-101] of 1940,

$$
\begin{aligned}
\frac{P}{\rho_{n} R T}=1 & +\left(B_{0}-\frac{A_{0}}{R T}-\frac{C_{0}}{R T^{3}}\right) \rho_{n}+\left(b-\frac{a}{R T}\right) \rho_{n}^{2} \\
& +\frac{\alpha a}{R T} \rho_{n}^{5}+\frac{c \rho_{n}^{2}}{R T^{3}}\left(1+\gamma \rho_{n}^{2}\right) \exp \left(-\gamma \rho_{n}^{2}\right),
\end{aligned}
$$

was an important step in the right direction and represented the volumetric properties of industrially important fluids (hydrocarbons) reasonably well. However, the BWR equation of state was found to give unsatisfactory results in low-temperature applications, at high fluid densities, and in the critical region. To alleviate these deficiencies, many modifications of the BWR equation, with many more parameters, have been proposed [56-58, 102-105] and are still widely used.

As already pointed out, fundamental equations have a great inherent advantage over volumetric PVTx equations of state: they contain complete information on the thermodynamic system. Thus, once a judiciously selected empirical relation has been developed for one of the fundamental equations, say, for $n U=U^{\mathrm{t}}\left(n S, n V,\left\{n_{i}\right\}\right)$, or $n H=H^{\mathrm{t}}\left(n S, P,\left\{n_{i}\right\}\right)$, or $n F=F^{\mathrm{t}}\left(T, n V,\left\{n_{i}\right\}\right)$, or $n G=G^{\mathrm{t}}\left(T, P,\left\{n_{i}\right\}\right)$, in principle, all other thermodynamic fluid properties can be calculated by combinations of appropriate derivatives. Since the independent variable entropy $n S$ is not directly measurable, neither the fundamental equation formulated in terms of internal energy, nor that formulated in terms of enthalpy, are used for developing empirical correlational equations. The advantage of using $n G=G^{\mathrm{t}}\left(T, P,\left\{n_{i}\right\}\right)$ is that the intensive canonical variables $T$ and $P$ are easily measured, monitored and controlled; this makes the Gibbs energy so important in Physical Chemistry as well as in Chemical Engineering. However, due to the discontinuity in slope of the Gibbs energy surface at the liquid (L)/vapor (V) phase boundary, i.e.,

$$
\rho_{n}^{\mathrm{L}}\left(T, P_{\sigma}\right)=1 / V^{\mathrm{L}}\left(T, P_{\sigma}\right) \neq \rho_{n}^{\mathrm{V}}\left(T, P_{\sigma}\right)=1 / V^{\mathrm{V}}\left(T, P_{\sigma}\right),
$$

$n G=G^{\mathrm{t}}\left(T, P,\left\{n_{i}\right\}\right)$ can only be used to represent the liquid part of the Gibbs energy surface or the vapor part separately, thus precluding a closed mathematical description of the entire fluid range ( $P=P_{\sigma}$ denotes the vapor pressure, with the subscript $\sigma$ indicating saturation conditions) $[63,64]$. In contradistinction, formulations based on Helmholtz energy-based fundamental equations, using canonical variables $T$ and $V$ (or $T$ and $\rho_{n}$ ), are suitable for describing the entire fluid region. They are valid for liquid and vapor states, for equilibria between them, and the description of supercritical states is included. Their validity range terminates at the melting curve, thus allowing the calculation of liquid-phase properties, but not of properties of the coexisting solid phase. Modern fundamental equations are usually based on the Helmholtz energy [63, 64, 106-108]. However, in practical applications the dimensionless property $F(T, V) / R T$ (see Eq. 73) is usually replaced by $F\left(T, \rho_{n}\right) / R T, \rho_{n}=1 / V$, which quantity is split into a residual part and a perfect-gas (ideal-gas) part, where both are empirically expressed by dimensionless functions of the 
inverse reduced temperature $\tau \equiv T_{\mathrm{c}} / T$ and the reduced amount density, i.e., the inverse reduced molar volume, $\delta \equiv \rho_{n} / \rho_{n, \mathrm{c}}=V_{\mathrm{c}} / V$ :

$$
\frac{F\left(T, \rho_{n}\right)}{R T}=\alpha(\tau, \delta)=\alpha^{\mathrm{r}}(\tau, \delta)+\alpha^{\mathrm{pg}}(\tau, \delta) .
$$

The commonly used functional form of the residual term is that found in modified BWR equations. For details, see Refs. [63, 64, 106-108]. The most important application of the approach represented by Eq. 187 is the IAPWS (International Association for the Properties of Water and Steam) formulation 1995 for the thermodynamic properties of water prepared by Wagner and Pruß [108]. By using high-precision experimental data and applying modern strategies for optimizing the functional form of the EOS and for the simultaneous nonlinear data fitting, they obtained the so-called IAPWS-95 formulation covering the temperature range from the melting line to $1273 \mathrm{~K}$ and pressures up to $1000 \mathrm{MPa}$. In this range, IAPWS-95 represents all data to within their experimental uncertainty. In the critical region, data are represented very well, and IAPWS-95 can be reasonably extrapolated up to extremely high pressures and temperatures. The equation for the dimensionless perfect-gas part $\alpha^{\mathrm{pg}}(\tau, \delta)$ contains 8 terms, while the equation for the dimensionless residual part $\alpha^{\mathrm{r}}(\tau, \delta)$ contains 56 terms.

Note that the dimensionless property $F(T, V) / R T$ is related to the molar Massieu function divided by $R$ (see Eq. 55):

$$
\frac{F}{R T}=-\frac{\Psi}{R}
$$

Quite recently, generalized models for calculating thermodynamic mixture properties using Helmholtz energy-based fundamental equations have been developed [109-113]. For instance, the GERG-2008 equation developed by Kunz and Wagner [113] is based on data of 21 natural gas components. Over the entire composition range, it covers the liquid phase, the gas phase, the supercritical region, and VLE within the range $90 \mathrm{~K}$ to $450 \mathrm{~K}$, and up to $35 \mathrm{MPa}$. However, when applied to significantly asymmetric binary mixtures, say, methane + pentane, it predicts critical curves with physically unreasonable temperature maxima [114]; thus, further work is indicated.

\subsection{Property Changes on Mixing and Excess Properties}

Experimental determination, correlation and prediction of mixture properties are topics of central importance in chemical thermodynamics, and data on binary liquid mixtures are of particular interest. Primarily, they are useful for testing and guiding theories that attempt to predict thermodynamic mixture properties from the properties of the constituent pure components, and the experimental results provide information on parameters characterizing interactions between unlike species. In turn, these data constitute the very foundation for the development of predictive methods for properties of liquid multicomponent mixtures that, on the application side, are indispensable for the calculation of phase equilibria. At present, no generally satisfactory theory exists that provides a reliable basis for prediction/correlation of thermodynamic data for binary liquid mixture, and thus, a fortiori, for ternary and multinary mixtures. Fortunately, large numbers of (critically) evaluated experimental data of excess properties (for a definition see below) are available in systematic data collections, such as the Solubility Data Series (IUPAC-NIST) [115, 116], NIST ThermoData Engine [117-120] or Landolt-Börnstein [121-129], or in data banks, such as 
the Dortmund Data Bank [70]. Based on this wealth of experimental information, wellhoned semi-empirical models, such as UNIFAC [130-136], DISQUAC [137-140], and the recent MOQUAC model [141] have been developed for correlating, extrapolating and predicting, in particular, molar excess Gibbs energies $G^{\mathrm{E}}$ and molar excess enthalpies $H^{\mathrm{E}}$, over reasonably large temperature ranges. However, estimated infinite-dilution properties, aqueous solubilities of hydrocarbons, and excess heat capacities $C_{P}^{\mathrm{E}}$ of liquid mixtures are frequently not satisfactory [142]. Similar comments apply to COSMO-RS and related models [143-147].

When describing reality, instead of considering total mixture properties $M^{\mathrm{t}}=n M\left(T, P,\left\{x_{i}\right\}\right)$ it is helpful to discuss the corresponding molar mixture properties in relation to the properties of the pure liquid constituents at the same $T, P$ and $\left\{x_{i}\right\}$, i.e., to focus on difference measures. Discussion is thus based on a new class of thermodynamic functions known as property changes of mixing, customarily designated by the symbol $\Delta$ and, on a molar basis, defined by

$$
\Delta M\left(T, P,\left\{x_{i}\right\}\right) \equiv M\left(T, P,\left\{x_{i}\right\}\right)-\sum_{i}^{c} x_{i} M_{i}^{*}(T, P) .
$$

The corresponding new class of partial molar property changes of mixing (see Eq. 52c) is defined by

$$
\left(\frac{\partial(n \Delta M)}{\partial n_{i}}\right)_{T, P, n_{j \neq i}} \equiv \Delta M_{i}\left(T, P,\left\{x_{i}\right\}\right)=M_{i}\left(T, P,\left\{x_{i}\right\}\right)-M_{i}^{*}(T, P) .
$$

With the corresponding summability relation at constant $T$ and $P$, see Eq. 52d, we have

$$
\Delta M\left(T, P,\left\{x_{i}\right\}\right)=\sum_{i}^{c} x_{i} \Delta M_{i}\left(T, P,\left\{x_{i}\right\}\right)
$$

or

$$
n \Delta M\left(T, P,\left\{x_{i}\right\}\right)=\sum_{i}^{c} n_{i} \Delta M_{i}\left(T, P,\left\{x_{i}\right\}\right),
$$

and the exact differential of the extensive property $n \Delta M$ is

$$
\mathrm{d}(n \Delta M)=n\left(\frac{\partial \Delta M}{\partial T}\right)_{P,\left\{x_{i}\right\}} \mathrm{d} T+n\left(\frac{\partial \Delta M}{\partial P}\right)_{T,\left\{x_{i}\right\}} \mathrm{d} P+\sum_{i}^{c} \Delta M_{i} \mathrm{~d} n_{i} .
$$

From Eq. 192 a differential change in $n \Delta M$ is given by

$$
\mathrm{d}(n \Delta M)=\sum_{i}^{c} \Delta M_{i} \mathrm{~d} n_{i}+\sum_{i}^{c} n_{i} \mathrm{~d} \Delta M_{i}
$$

Hence, comparison with Eq. 193, and after division by $n$,

$$
\left(\frac{\partial \Delta M}{\partial T}\right)_{P,\left\{x_{i}\right\}} \mathrm{d} T+\left(\frac{\partial \Delta M}{\partial P}\right)_{T,\left\{x_{i}\right\}} \mathrm{d} P-\sum_{i}^{c} x_{i} \mathrm{~d} \Delta M_{i}=0,
$$


is obtained: this is another form of the general Gibbs-Duhem equation. In this section, the focus will be on $M=G, S, V, H$, and because of direct measurability, $\Delta H$ (via calorimetry) and $\Delta V$ (via dilatometry) are the molar property changes of mixing of special interest.

Applying residual-function approaches or equations-of-state methods to liquid mixtures, frequently consisting of chemically quite complex components, is often unsatisfactory. Thus, for many applications discussed in this symposium, one way to proceed is to select in place of the perfect gas (ideal gas) a reference basis that is more appropriate for condensed phases: the most commonly selected basis is the ideal solution (indicated by a superscript id). In fact, this approach represents the classic method in solution thermodynamics [148-150]. Compared to the perfect-gas basis, we recognize that changes in composition affect liquid mixture properties much stronger than changes in pressure.

Consider a liquid equilibrium phase with composition $\left\{x_{i}\right\} \equiv\left\{x_{i}^{\mathrm{L}}\right\}$ at uniform temperature and pressure. Using the idealized composition dependence of the component fugacity as represented by the Lewis-Randall (LR) rule [17, 66, 151],

$$
f_{i}^{\mathrm{id}}\left(T, P,\left\{x_{i}\right\}\right) \equiv f_{i}^{\mathrm{LR}}\left(T, P,\left\{x_{i}\right\}\right)=x_{i} f_{i}^{\mathrm{L}, *}(T, P), \text { valid for } 0 \leq x_{i} \leq 1,
$$

one obtains for the partial molar Gibbs energy.

$$
G_{i}^{\mathrm{id}}\left(T, P,\left\{x_{i}\right\}\right)=G_{i}^{*}(T, P)+R T \ln x_{i} .
$$

Discussion of real-solution behavior may now be based on deviations from Lewis-Randall ideal-solution behavior, i.e., on the differences between property values of real solutions and property values calculated for the Lewis-Randall ideal-solution model at the same T, $P$ and $\left\{x_{i}\right\}$ based on Eq. 197. That is, the partial molar Gibbs energy $G_{i}^{\text {id }}$ of component $i$ serves as a generating function for other partial molar properties of an LR-ideal solution that hence-forward will be indicated by a superscript id (alternative ideal-solution models are possible, and are indeed used, though the LR-ideal solution is the conventional reference for mixture behavior of liquid phases). For instance, the temperature derivative and the pressure derivative yield the partial molar entropy and the partial molar volume, respectively,

$$
\begin{gathered}
S_{i}^{\mathrm{id}}\left(T, P,\left\{x_{i}\right\}\right)=S_{i}^{*}(T, P)-R \ln x_{i}, \\
V_{i}^{\mathrm{id}}\left(T, P,\left\{x_{i}\right\}\right)=V_{i}^{*}(T, P),
\end{gathered}
$$

while the Gibbs-Helmholtz equation yields the LR-ideal partial molar enthalpy

$$
H_{i}^{\mathrm{id}}\left(T, P,\left\{x_{i}\right\}\right)=H_{i}^{*}(T, P) .
$$

The LR-ideal molar properties corresponding to the partial molar properties of Eqs. 197-200 are obtained with the summability relation:

$$
\begin{gathered}
G^{\mathrm{id}}\left(T, P,\left\{x_{i}\right\}\right)=\sum_{i} x_{i} G_{i}^{*}(T, P)+R T \sum_{i} x_{i} \ln x_{i}, \\
S^{\mathrm{id}}\left(T, P,\left\{x_{i}\right\}\right)=\sum_{i} x_{i} S_{i}^{*}(T, P)-R \sum_{i} x_{i} \ln x_{i},
\end{gathered}
$$




$$
\begin{gathered}
V^{\text {id }}\left(T, P,\left\{x_{i}\right\}\right)=\sum_{i} x_{i} V_{i}^{*}(T, P), \\
H^{\text {id }}\left(T, P,\left\{x_{i}\right\}\right)=\sum_{i} x_{i} H_{i}^{*}(T, P) .
\end{gathered}
$$

The molar property changes of mixing for LR-ideal solutions, $\Delta M^{\mathrm{id}}$, may be obtained as special cases from the general defining equation, Eq. 189:

$$
\left.\begin{array}{rl}
\Delta M^{\mathrm{id}}\left(T, P,\left\{x_{i}\right\}\right) \equiv & M^{\mathrm{id}}\left(T, P,\left\{x_{i}\right\}\right)-\sum_{i} x_{i} M_{i}^{*}(T, P)=\sum_{i} x_{i}\left[M_{i}^{\mathrm{id}}\left(T, P,\left\{x_{i}\right\}\right)-M_{i}^{*}(T, P)\right] \\
& =\sum_{i} x_{i} \Delta M_{i}^{\mathrm{id}}\left(T, P,\left\{x_{i}\right\}\right)
\end{array}\right\}
$$

That is, by substituting the corresponding expression for $M^{\text {id }}$, Eqs. 201-204, into Eq. 205 (or the corresponding expressions for $M_{i}^{\text {id }}$, Eqs. 197-200), we obtain

$$
\begin{gathered}
\Delta G^{\mathrm{id}}\left(T, P,\left\{x_{i}\right\}\right)=\sum_{i} x_{i} \Delta G_{i}^{\mathrm{id}}\left(T, P,\left\{x_{i}\right\}\right)=R T \sum_{i} x_{i} \ln x_{i}, \\
\Delta S^{\mathrm{id}}\left(T, P,\left\{x_{i}\right\}\right)=\sum_{i} x_{i} \Delta S_{i}^{\mathrm{id}}\left(T, P,\left\{x_{i}\right\}\right)=-R \sum_{i} x_{i} \ln x_{i} . \\
\Delta V^{\mathrm{id}}\left(T, P,\left\{x_{i}\right\}\right)=\sum_{i} x_{i} \Delta V_{i}^{\mathrm{id}}\left(T, P,\left\{x_{i}\right\}\right)=0, \\
\Delta H^{\mathrm{id}}\left(T, P,\left\{x_{i}\right\}\right)=\sum_{i} x_{i} \Delta H_{i}^{\mathrm{id}}\left(T, P,\left\{x_{i}\right\}\right)=0 .
\end{gathered}
$$

The general property $\Delta M_{i}^{\text {id }}\left(T, P,\left\{x_{i}\right\}\right)$ of Eq. 205 denotes a partial molar property change of mixing for LR-ideal solutions, such as those appearing in Eqs. 206-209:

$$
\Delta M_{i}^{\mathrm{id}}\left(T, P,\left\{x_{i}\right\}\right) \equiv M_{i}^{\mathrm{id}}\left(T, P,\left\{x_{i}\right\}\right)-M_{i}^{*}(T, P)
$$

Quantities measuring deviations of real solution properties $M\left(T, P,\left\{x_{i}\right\}\right)$ from LR-ideal solution properties $M^{\mathrm{id}}\left(T, P,\left\{x_{i}\right\}\right)$ at the same $T, P$ and $\left\{x_{i}\right\}$ (see Eqs. 201-204), constitute another highly useful new class of functions called molar excess properties. They are designated by a superscript $\mathrm{E}$ and are defined by

$$
M^{\mathrm{E}}\left(T, P,\left\{x_{i}\right\}\right) \equiv M\left(T, P,\left\{x_{i}\right\}\right)-M^{\mathrm{id}}\left(T, P,\left\{x_{i}\right\}\right) .
$$

The corresponding partial molar excess properties for component $i$ in solution are defined by

$$
\left(\frac{\partial\left(n M^{\mathrm{E}}\right)}{\partial n_{i}}\right)_{T, P, n_{j \neq i}} \equiv M_{i}^{\mathrm{E}}\left(T, P,\left\{x_{i}\right\}\right)=M_{i}\left(T, P,\left\{x_{i}\right\}\right)-M_{i}^{\mathrm{id}}\left(T, P,\left\{x_{i}\right\}\right),
$$

and with the summability relation we have 


$$
M^{\mathrm{E}}\left(T, P,\left\{x_{i}\right\}\right)=\sum_{i}^{c} x_{i} M_{i}^{\mathrm{E}}\left(T, P,\left\{x_{i}\right\}\right) .
$$

Substituting the ideal-solution expressions Eqs. 201-204 into Eq. 211, and taking into account Eq. 189, we obtain the following relations:

$$
\begin{gathered}
\left.G^{\mathrm{E}}\left(T, P,\left\{x_{i}\right\}\right)=G\left(T, P,\left\{x_{i}\right\}\right)-\sum_{i}^{c} x_{i} G_{i}^{*}(T, P)-R T \sum_{i}^{c} x_{i} \ln x_{i}\right\} \\
\left.=\Delta G\left(T, P,\left\{x_{1}\right\}\right)-R T \sum_{i}^{c} x_{i} \ln x_{i}\right\}, \\
\left.S^{\mathrm{E}}\left(T, P,\left\{x_{i}\right\}\right)=S\left(T, P,\left\{x_{i}\right\}\right)-\sum_{i}^{c} x_{i} S_{i}^{*}(T, P)+R \sum_{i}^{c} x_{i} \ln x_{i}\right\}, \\
\left.=\Delta S\left(T, P,\left\{x_{i}\right\}\right)+R \sum_{i}^{c} x_{i} \ln x_{i}\right\} \\
V^{\mathrm{E}}\left(T, P,\left\{x_{i}\right\}\right)=V\left(T, P,\left\{x_{i}\right\}\right)-\sum_{i}^{c} x_{i} V_{i}^{*}(T, P) \\
=\Delta V\left(T, P,\left\{x_{i}\right\}\right) \\
H^{\mathrm{E}}\left(T, P,\left\{x_{i}\right\}\right)=H\left(T, P,\left\{x_{i}\right\}\right)-\sum_{i}^{c} x_{i} H_{i}^{*}(T, P) \\
=\Delta H\left(T, P,\left\{x_{i}\right\}\right)
\end{gathered}
$$

First-law excess properties are identical to the property changes of mixing.

As a generating function, the molar excess Gibbs energy $G^{\mathrm{E}}$ is of central interest. As a matter of convenience, Eq. 197 may be generalized in such a manner that an expression for the partial molar Gibbs energy $G_{i}$ is obtained that is valid for any real mixture per definition. Thus, we may write

$$
\left.\begin{array}{rl}
G_{i}\left(T, P,\left\{x_{i}\right\}\right) & \equiv G_{i}^{*}(T, P)+R T \ln x_{i}+R T \ln \gamma_{i}^{\mathrm{LR}}\left(T, P,\left\{x_{i}\right\}\right) \\
& =G_{i}^{\mathrm{id}}\left(T, P,\left\{x_{i}\right\}\right)+R T \ln \gamma_{i}^{\mathrm{LR}}\left(T, P,\left\{x_{i}\right\}\right)
\end{array}\right\},
$$

where $\gamma_{i}^{\mathrm{LR}}\left(T, P,\left\{x_{i}\right\}\right)$ is known as the Lewis-Randall (LR) activity coefficient of component $i$ in solution. With the defining prescription Eq. 212, the partial molar excess Gibbs energy is thus given by

$$
\left.\begin{array}{rl}
\left(\frac{\partial\left(n G^{\mathrm{E}}\right)}{\partial n_{i}}\right)_{T, P, n_{j \neq i}} & \equiv G_{i}^{\mathrm{E}}\left(T, P,\left\{x_{i}\right\}\right)=G_{i}\left(T, P,\left\{x_{i}\right\}\right)-G_{i}^{\mathrm{id}}\left(T, P,\left\{x_{i}\right\}\right) \\
& =R T \ln \gamma_{i}^{\mathrm{LR}}\left(T, P,\left\{x_{i}\right\}\right)
\end{array}\right\} .
$$

In view of Eq. 213, the molar excess Gibbs energy reads 


$$
\left.\begin{array}{rl}
G^{\mathrm{E}}\left(T, P,\left\{x_{i}\right\}\right) & =G\left(T, P,\left\{x_{i}\right\}\right)-G^{\mathrm{id}}\left(T, P,\left\{x_{i}\right\}\right)=\sum_{i}^{c} x_{i} G_{i}^{\mathrm{E}}\left(T, P,\left\{x_{i}\right\}\right) \\
& =R T \sum_{i}^{c} x_{i} \ln \gamma_{i}^{\mathrm{LR}}\left(T, P,\left\{x_{i}\right\}\right)
\end{array}\right\}
$$

Since $S_{i}=-\left(\partial G_{i} / \partial T\right)_{P,\left\{x_{i}\right\}}$, we have for the molar excess entropy

$$
\begin{aligned}
S^{\mathrm{E}}\left(T, P,\left\{x_{i}\right\}\right) & =S\left(T, P,\left\{x_{i}\right\}\right)-S^{\mathrm{id}}\left(T, P,\left\{x_{i}\right\}\right)=\sum_{i}^{c} x_{i} S_{i}^{\mathrm{E}}\left(T, P,\left\{x_{i}\right\}\right) \\
& \left.=-R \sum_{i}^{c} x_{i} \ln \gamma_{i}^{\mathrm{LR}}\left(T, P,\left\{x_{i}\right\}\right)-R T \sum_{i}^{c} x_{i}\left(\partial \ln \gamma_{i}^{\mathrm{LR}}\left(T, P,\left\{x_{i}\right\}\right) / \partial T\right)_{P,\left\{x_{i}\right\}}\right\}
\end{aligned}
$$

With $V_{i}=\left(\partial G_{i} / \partial P\right)_{T,\left\{x_{i}\right\}}$, the molar excess volume is given by

$$
\begin{aligned}
V^{\mathrm{E}}\left(T, P,\left\{x_{i}\right\}\right) & \left.=V\left(T, P,\left\{x_{i}\right\}\right)-V^{\mathrm{id}}\left(T, P,\left\{x_{i}\right\}\right)=\sum_{i}^{c} x_{i} V_{i}^{\mathrm{E}}\left(T, P,\left\{x_{i}\right\}\right)\right\} \\
& \left.=R T \sum_{i}^{c} x_{i}\left(\partial \ln \gamma_{i}^{\mathrm{LR}}\left(T, P,\left\{x_{i}\right\}\right) / \partial P\right)_{T,\left\{x_{i}\right\}}\right\},
\end{aligned}
$$

and finally, with the Gibbs-Helmholtz equation or via $H^{\mathrm{E}}=G^{\mathrm{E}}+T S^{\mathrm{E}}$ we obtain for the molar excess enthalpy

$$
\begin{aligned}
H^{\mathrm{E}}\left(T, P,\left\{x_{i}\right\}\right) & \left.=H\left(T, P,\left\{x_{i}\right\}\right)-H^{\mathrm{id}}\left(T, P,\left\{x_{i}\right\}\right)=\sum_{i}^{c} x_{i} H_{i}^{\mathrm{E}}\left(T, P,\left\{x_{i}\right\}\right)\right\} \\
& \left.=-R T^{2} \sum_{i}^{c} x_{i}\left(\partial \ln \gamma_{i}^{\mathrm{LR}}\left(T, P,\left\{x_{i}\right\}\right) / \partial T\right)_{P,\left\{x_{i}\right\}}\right\} .
\end{aligned}
$$

The ideal-solution equations for $M^{\text {id }}(M=G, S, V, H)$ which follow from the Lewis-Randall rule are given by Eqs. 201-204. I reiterate that Eqs. 220-223 only apply when the Lewis-Randall model for the ideal solution is used.

Central to the development of useful relations is the fundamental property relation

$$
\mathrm{d}\left(n G^{\mathrm{E}}\right)=-\left(n S^{\mathrm{E}}\right) \mathrm{d} T+\left(n V^{\mathrm{E}}\right) \mathrm{d} P+R T \sum_{i}^{c} \ln \gamma_{i}^{\mathrm{LR}} \mathrm{d} n_{i},
$$

and, of course,

$$
G^{\mathrm{E}}=H^{\mathrm{E}}-T S^{\mathrm{E}} \text { and } G_{i}^{\mathrm{E}}=H_{i}^{\mathrm{E}}-T S_{i}^{\mathrm{E}},
$$

and so forth. The definition of an excess property is not restricted to any phase, though they are predominantly used for liquid mixtures.

Excess properties and property changes of mixing are closely related, and one may readily calculate $M^{\mathrm{E}}$ from $\Delta M$ and vice versa. By combining the definitions Eqs. 211 and 189, in conjunction with $\Delta M^{\text {id }}$ defined by Eq. 205 , the important relation 


$$
M^{\mathrm{E}}\left(T, P,\left\{x_{i}\right\}\right)=\Delta M\left(T, P,\left\{x_{i}\right\}\right)-\Delta M^{\mathrm{id}}\left(T, P,\left\{x_{i}\right\}\right),
$$

is obtained, with a similar one holding for the corresponding partial molar quantities:

$$
M_{i}^{\mathrm{E}}\left(T, P,\left\{x_{i}\right\}\right)=\Delta M_{i}\left(T, P,\left\{x_{i}\right\}\right)-\Delta M_{i}^{\mathrm{id}}\left(T, P,\left\{x_{i}\right\}\right) .
$$

Rearranging Eq. 226, the difference $\Delta M-M^{\mathrm{E}}=\Delta M^{\text {id }}$ is zero except for the secondlaw properties $M=G, F(=G-P V)$ and $S$, see Eqs. 206-209, and similarly for the partial molar properties in Eq. 227, the difference $\Delta M_{i}-M_{i}^{\mathrm{E}}=\Delta M_{i}^{\mathrm{id}}$ is zero except for the second-law properties $M_{i}=G_{i}, F_{i}\left(=G_{i}-P V_{i}\right)$ and $S_{i}$. Further, from Eq. 226 we see immediately that since a molar excess property represents also the difference between the real change of a property of mixing and the LR-ideal-solution change of a property of mixing, we may identify it, alternatively to the defining equation Eq. 211, as a molar excess property change of mixing

$$
M^{\mathrm{E}}=\Delta M-\Delta M^{\mathrm{id}} \equiv(\Delta M)^{\mathrm{E}} .
$$

Analogously, from Eq. 228 we may identify, alternatively to defining equation Eq. 212, a partial molar excess property as a partial molar excess property change of mixing

$$
M_{i}^{\mathrm{E}}=\Delta M_{i}-\Delta M_{i}^{\mathrm{id}} \equiv\left(\Delta M_{i}\right)^{\mathrm{E}} .
$$

Evidently, the terms molar excess property and molar excess property change of mixing may be used interchangeably, and both are indeed found in the literature. If the focus is on properties of mixtures, then $M^{\mathrm{E}}$ and $M_{i}^{\mathrm{E}}$ are preferred, while for mixing processes the notations $(\Delta M)^{\mathrm{E}}=\Delta M^{\mathrm{E}}$ and $\left(\Delta M_{i}\right)^{\mathrm{E}}=\Delta M_{i}^{\mathrm{E}}$ may be regarded as being more appropriate.

For the four quantities selected for a more detailed discussion, Eqs. 226 and 227 yield the following: for the second-law properties

$$
\begin{gathered}
G^{\mathrm{E}}=\Delta G-R T \sum_{i}^{c} x_{i} \ln x_{i}, \text { and } G_{i}^{\mathrm{E}}=\Delta G_{i}-R T \ln x_{i}, \\
S^{\mathrm{E}}=\Delta S+R \sum_{i}^{c} x_{i} \ln x_{i}, \text { and } S_{i}^{\mathrm{E}}=\Delta S_{i}+R \ln x_{i},
\end{gathered}
$$

is obtained, while for the first-law properties we have

$$
\begin{gathered}
V^{\mathrm{E}}=\Delta V, \text { and } V_{i}^{\mathrm{E}}=\Delta V_{i}, \\
H^{\mathrm{E}}=\Delta H, \text { and } H_{i}^{\mathrm{E}}=\Delta H_{i},
\end{gathered}
$$

in accord with Eqs. 214-217. Depending on the point of view, $V^{\mathrm{E}}=\Delta V$ is known as either the molar excess volume or the molar volume change of mixing, and $H^{\mathrm{E}}=\Delta H$ is called either the molar excess enthalpy or the molar enthalpy change of mixing.

In analogy to Eq. 193, the exact differential of the extensive property $n M^{\mathrm{E}}\left(T, P,\left\{x_{i}\right\}\right)$ is given by

$$
\mathrm{d}\left(n M^{\mathrm{E}}\right)=n\left(\frac{\partial M^{\mathrm{E}}}{\partial T}\right)_{P,\left\{x_{i}\right\}} \mathrm{d} T+n\left(\frac{\partial M^{\mathrm{E}}}{\partial P}\right)_{T,\left\{x_{i}\right\}} \mathrm{d} P+\sum_{i}^{c} M_{i}^{\mathrm{E}} \mathrm{d} n_{i},
$$


while Eq. 213 yields for a differential change in $n M^{\mathrm{E}}$

$$
\mathrm{d}\left(n M^{\mathrm{E}}\right)=\sum_{i}^{c} M_{i}^{\mathrm{E}} \mathrm{d} n_{i}+\sum_{i}^{c} n_{i} \mathrm{~d} M_{i}^{\mathrm{E}} .
$$

Comparison with Eq. 234 and division by $n$ results in

$$
\left(\frac{\partial M^{\mathrm{E}}}{\partial T}\right)_{P,\left\{x_{i}\right\}} \mathrm{d} T+\left(\frac{\partial M^{\mathrm{E}}}{\partial P}\right)_{T,\left\{x_{i}\right\}} \mathrm{d} P-\sum_{i}^{c} x_{i} \mathrm{~d} M_{i}^{\mathrm{E}}=0,
$$

which is still another form of the general Gibbs-Duhem equation.

For convenience, instead of $G_{i}^{\mathrm{E}}$ the non-dimensional group $G_{i}^{\mathrm{E}} / R T$ is frequently used, which quantity is directly related to the LR-based dimensionless state function $\ln \gamma_{i}^{\mathrm{LR}}\left(T, P,\left\{x_{i}\right\}\right)$ by

$$
G_{i}^{\mathrm{E}} / R T \equiv \mu_{i}^{\mathrm{E}} / R T=\ln \gamma_{i}^{\mathrm{LR}}
$$

Using the summability relation, we have

$$
\begin{aligned}
\frac{G^{\mathrm{E}}\left(T, P,\left\{x_{i}\right\}\right)}{R T} & =\sum_{i}^{c} x_{i} \frac{G_{i}^{\mathrm{E}}\left(T, P,\left\{x_{i}\right\}\right)}{R T} \\
& =\sum_{i}^{c} x_{i} \frac{\mu_{i}^{\mathrm{E}}\left(T, P,\left\{x_{i}\right\}\right)}{R T}=\sum_{i}^{c} x_{i} \ln \gamma_{i}^{\mathrm{LR}}\left(T, P,\left\{x_{i}\right\}\right) .
\end{aligned}
$$

The corresponding fundamental excess-property relation for a single-phase system, in which the amounts $n_{i}$ may vary either through interchange of matter with its surroundings (open phase) or because of chemical reactions within the system or both, reads

$$
\left.\mathrm{d}\left(\frac{n G^{\mathrm{E}}}{R T}\right)=-\frac{n H^{\mathrm{E}}}{R T^{2}} \mathrm{~d} T+\frac{n V^{\mathrm{E}}}{R T} \mathrm{~d} P+\sum_{i}^{c} \frac{G_{i}^{\mathrm{E}}}{R T} \mathrm{~d} n_{i}=-\frac{n H^{\mathrm{E}}}{R T^{2}} \mathrm{~d} T+\frac{n V^{\mathrm{E}}}{R T} \mathrm{~d} P+\sum_{i}^{c} \ln \gamma_{i}^{\mathrm{LR}} \mathrm{d} n_{i}\right\},
$$

in complete analogy to Eq. 70. By inspection we find

$$
\begin{gathered}
\left(\frac{\partial\left(G^{\mathrm{E}} / R T\right)}{\partial T}\right)_{P,\left\{x_{i}\right\}}=-\frac{H^{\mathrm{E}}}{R T^{2}}, \\
\left(\frac{\partial\left(G^{\mathrm{E}} / R T\right)}{\partial P}\right)_{T,\left\{x_{i}\right\}}=\frac{V^{\mathrm{E}}}{R T}, \\
\left(\frac{\partial\left(n G^{\mathrm{E}} / R T\right)}{\partial n_{i}}\right)_{T, P, n_{j \neq i}}=\frac{G_{i}^{\mathrm{E}}}{R T}=\ln \gamma_{i}^{\mathrm{LR}} .
\end{gathered}
$$

The corresponding Gibbs-Duhem equation reads 


$$
-\frac{H^{\mathrm{E}}}{R T^{2}} \mathrm{~d} T+\frac{V^{\mathrm{E}}}{R T} \mathrm{~d} P-\sum_{i}^{c} x_{i} \mathrm{~d} \ln \gamma_{i}^{\mathrm{LR}}=0,
$$

which at constant $T$ and $P$ reduces to

$$
\sum_{i}^{c} x_{i} \mathrm{~d} \ln \gamma_{i}^{\mathrm{LR}}\left(T, P,\left\{x_{i}\right\}\right)=0 .
$$

Rewriting it for a binary mixture

$$
\frac{\mathrm{d} \ln \gamma_{1}^{\mathrm{LR}}}{\mathrm{d} x_{1}}=-\frac{x_{2}}{x_{1}} \frac{\mathrm{d} \ln \gamma_{2}^{\mathrm{LR}}}{\mathrm{d} x_{1}}
$$

we recognize that if in a binary mixture $\gamma_{1}^{\mathrm{LR}}$ increases (or decreases) with increasing $x_{1}$, then $\gamma_{2}^{\mathrm{LR}}$ must simultaneously decrease (or increase) with increasing $x_{1}$. In addition, when $x_{2} \rightarrow 0$, and thus $x_{1} \rightarrow 1$, the slope of the curve $\ln \gamma_{1}^{\mathrm{LR}}$ vs. $x_{1}$ is zero, and vice versa, when $x_{1} \rightarrow 0$, and thus $x_{2} \rightarrow 1$, the slope of the curve $\ln \gamma_{2}^{\mathrm{LR}}$ vs. $x_{1}$ is zero: each $\ln \gamma_{i}^{\mathrm{LR}}$ curve $(i=1,2)$ terminates at $x_{i}^{\mathrm{LR}}=1$ with zero value and zero slope.

The fundamental excess-property relation Eq. 239 with canonical variables $T, P$ and $\left\{n_{i}\right\}$ supplies complete information on excess properties. It is of central importance in solution chemistry because $H^{\mathrm{E}}$, and its temperature dependence $C_{P}^{\mathrm{E}}$, the molar excess heat capacity at constant pressure (molar excess isobaric heat capacity)

$$
\left.\begin{array}{rl}
C_{P}^{\mathrm{E}}\left(T, P,\left\{x_{i}\right\}\right) & \left.=\left(\frac{\partial H^{\mathrm{E}}\left(T, P,\left\{x_{i}\right\}\right)}{\partial T}\right)_{P,\left\{x_{i}\right\}}=T\left(\frac{\partial S^{\mathrm{E}}\left(T, P,\left\{x_{i}\right\}\right)}{\partial T}\right)_{P,\left\{x_{i}\right\}}\right\}, \\
& =C_{P}\left(T, P,\left\{x_{i}\right\}\right)-\sum_{i}^{c} x_{i} C_{P_{i}}^{*}(T, P)=\Delta C_{P}\left(T, P,\left\{x_{i}\right\}\right)
\end{array}\right\}
$$

the molar excess volume $V^{\mathrm{E}}$ and $\ln \gamma_{i}^{\mathrm{LR}}$ are experimentally accessible quantities: excess enthalpies and excess isobaric heat capacities may be obtained more or less directly via calorimetry, and excess volumes may be obtained more or less directly via dilatometry, or pycnometry, or vibrating-tube densimetry, or buoyancy methods (exploiting the Archimedes principle), respectively [37, 46, 66, 68, 152-160]. The excess Gibbs energy, in principle the key excess property, is a conceptual property that cannot be measured directly (nor can the excess entropy), though it can be quantitatively deduced from vapor-liquid equilibrium (VLE) measurements via determination of LR activity coefficients [17, 20, 46, 161-172]. For mixtures at low to moderate pressure, that is, well below the critical pressure, the conventional highly effective and refined "phi/gamma $(\phi / \gamma)$ ") approach to VLE is commonly used: note that isothermal measurements are advantageous [17]. For applications at pressures up to a few bars, to an excellent approximation the equilibrium relation for a binary system reads

$$
y_{i} P \Phi_{i}=x_{i} \gamma_{i}^{\mathrm{LR}} P_{\sigma, i}, \text { hence } \gamma_{i}^{\mathrm{LR}}=\frac{y_{i} P \Phi_{i}}{x_{i} P_{\sigma, i}}, i=1 \text { or } 2,
$$

and 


$$
\begin{gathered}
\Phi_{i}=\frac{\phi_{i}^{\mathrm{V}}\left(T, P, y_{i}\right)}{\phi_{i}^{\mathrm{V}, *}\left(T, P_{\sigma, i}\right)} \exp \left[-\int_{P_{\sigma, i}}^{P} \frac{V_{i}^{\mathrm{L}, *}}{R T} \mathrm{~d} P\right], \\
\cong \exp \left[\frac{\left(B_{i i}-V_{i}^{\mathrm{L}, *}\right)\left(P-P_{\sigma, i}\right)+P y_{j}^{2} \delta_{i j}}{R T}\right], i \neq j .
\end{gathered}
$$

Here, $\delta_{i j}$ is defined by

$$
\delta_{i j} \equiv 2 B_{i j}-B_{i i}-B_{j j}
$$

where the identically subscripted second virial coefficients $B_{i i}$ and $B_{j j}$ refer to the pure vapor of component $i$ or $j$, respectively, and $B_{i j}$ is known as the interaction virial coefficient, or cross-coefficient. Note that we have $\delta_{i j}=\delta_{j i}$. In Eq. $247 \mathrm{a}, \phi_{i}^{\mathrm{V}}\left(T, P, y_{i}\right)$ denotes the vapor-phase fugacity coefficient of component $i$, and $\phi_{i}^{\mathrm{V}, *}\left(T, P_{\sigma, i}\right)$ is the fugacity coefficient of pure saturated vapor at $T$ and $P_{\sigma, i}$. These phase-equilibrium relations are easily extended to multicomponent mixtures.

The classical data reduction approach uses activity coefficients directly determined by Eq. 246. Insertion of the so obtained $\gamma_{i}^{\mathrm{LR}} \mathrm{s}$ into Eq. 220 or Eq. 238 yields values of $G^{\mathrm{E}} / R T=\sum_{i}^{c} x_{i} \ln \gamma_{i}^{\mathrm{LR}}$ that are then fit to an appropriate analytical correlating equation representing the composition dependence of $G^{\mathrm{E}} / R T$ (see next section). For more recent methods see Van Ness and Abbott [17]. Combination with calorimetrically measured excess enthalpies yields the molar excess entropy

$$
S^{\mathrm{E}}\left(T, P,\left\{x_{i}\right\}\right)=\left[H^{\mathrm{E}}\left(T, P,\left\{x_{i}\right\}\right)-G^{\mathrm{E}}\left(T, P,\left\{x_{i}\right\}\right)\right] / T .
$$

For one mole of a constant-composition mixture,

$$
\mathrm{d}\left(\frac{G^{\mathrm{E}}}{R T}\right)=-\frac{H^{\mathrm{E}}}{R T^{2}} \mathrm{~d} T+\frac{V^{\mathrm{E}}}{R T} \mathrm{~d} P,
$$

and for the corresponding partial molar excess properties, see Eq. 237,

$$
\mathrm{d}\left(\frac{G_{i}^{\mathrm{E}}}{R T}\right)=\mathrm{d} \ln \gamma_{i}^{\mathrm{LR}}=-\frac{H_{i}^{\mathrm{E}}}{R T^{2}} \mathrm{~d} T+\frac{V_{i}^{\mathrm{E}}}{R T} \mathrm{~d} P,
$$

is obtained. Hence the partial molar analogues of Eqs. 240 and 241, respectively, are

$$
\left(\frac{\partial \ln \gamma_{i}^{\mathrm{LR}}}{\partial T}\right)_{P,\left\{x_{i}\right\}}=-\frac{H_{i}^{\mathrm{E}}}{R T^{2}}
$$

and

$$
\left(\frac{\partial \ln \gamma_{i}^{\mathrm{LR}}}{\partial P}\right)_{T,\left\{x_{i}\right\}}=\frac{V_{i}^{\mathrm{E}}}{R T}
$$

Thus, the partial molar excess entropy is given by 


$$
S_{i}^{\mathrm{E}}=\frac{H_{i}^{\mathrm{E}}-G_{i}^{E}}{T}=-R T\left(\frac{\partial \ln \gamma_{i}^{\mathrm{LR}}}{\partial T}\right)_{P,\left\{x_{i}\right\}}-R \ln \gamma_{i}^{\mathrm{LR}}
$$

Finally, I list the useful relations

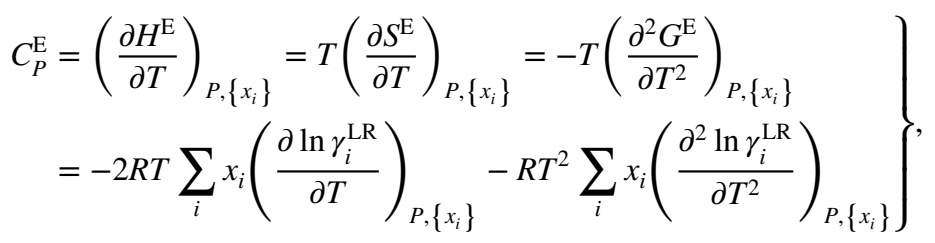

and, important for calorimetric work at high pressure

$$
\left(\frac{\partial H^{\mathrm{E}}}{\partial P}\right)_{T,\left\{x_{i}\right\}}=V^{\mathrm{E}}-T\left(\frac{\partial V^{\mathrm{E}}}{\partial T}\right)_{P,\left\{x_{i}\right\}}=\left[\frac{\partial\left(V^{\mathrm{E}} / T\right)}{\partial(1 / T)}\right]_{P,\left\{x_{i}\right\}},
$$

and

$$
\left(\partial C_{P}^{\mathrm{E}} / \partial P\right)_{T,\left\{x_{i}\right\}}=-T\left(\partial^{2} V^{\mathrm{E}} / \partial T^{2}\right)_{P,\left\{x_{i}\right\}} \cdot
$$

Modern calorimeters allow reliable measurements of $H^{\mathrm{E}}$ and $C_{P}$ (and thus of $C_{P}^{\mathrm{E}}$ ) at elevated $T$ and $P$, and the results have to be consistent with experimentally determined volumetric properties, as indicated by Eqs. 256 and 257, respectively. However, outside the critical region the influence of pressure on excess properties is usually rather small.

Focusing now on the non-dimensional excess property $G^{\mathrm{E}} /\left(x_{1} x_{2} R T\right)$ for a binary mixture, we find this quantity of considerable practical utility, especially when a graphical (visual) evaluation of experimentally determined $G^{\mathrm{E}} \mathrm{s}$ is intended. Note that

$$
\lim _{x_{1} \rightarrow 0} \frac{G^{\mathrm{E}} / R T}{x_{1} x_{2}}=\ln \gamma_{1}^{\mathrm{LR}, \infty} \text { and } \lim _{x_{2} \rightarrow 0} \frac{G^{\mathrm{E}} / R T}{x_{1} x_{2}}=\ln \gamma_{2}^{\mathrm{LR}, \infty},
$$

where $\gamma_{1}^{\mathrm{LR}, \infty}$ and $\gamma_{2}^{\mathrm{LR}, \infty}$ are the LR activity coefficients at infinite dilution. These quantities play an important role in solution chemistry and have found many applications in the characterization of liquid solution behavior. In general, for binary mixtures extrapolation of $M^{\mathrm{E}} / x_{1} x_{2}$ to $x_{1}=0$ and $x_{2}=0$, respectively, is the most convenient and reliable graphical method for determining the infinite-dilution partial molar excess properties $M_{1}^{\mathrm{E}, \infty}$ and $M_{2}^{\mathrm{E}, \infty}$.

\subsection{Correlation of Experimental Data}

In general, experimental data have to be treated on their way from experiment in the laboratory to the place of application, be it in support of theory development, or model checking, or plant operation. Clearly, the mathematical treatment should aim to eliminate inconsistencies without distorting the results, it should correlate the data to within experimental error, provide at least realistic first derivatives upon differentiation, and it should combine adequate flexibility with reasonable simplicity. Unfortunately, no theoretical model of any general validity exists that satisfactorily describes the composition dependence of excess properties of liquid mixtures, and relations commonly used are semiempirical at best [1, 
16-18]. Focusing now on binary liquid mixtures, one procedure is to express $M^{\mathrm{E}} / x_{1} x_{2}$ at constant temperature and pressure as a power series in $x_{1}$ (since $x_{2}=1-x_{1}, x_{1}$ can be selected as the single independent variable). However, an equivalent power series, with certain advantages, is the popular empirical expansion due to Redlich and Kister [173-175],

$$
\frac{M^{\mathrm{E}}}{x_{1} x_{2}}=A_{0}+\sum_{m=1}^{a} A_{m}\left(x_{1}-x_{2}\right)^{m}, \text { constant } T, P,
$$

where the parameters $A_{0}$ and $A_{m}, m=1,2,3, \ldots$ are, in general, functions of $T$ and $P$; truncations of Eq. 259 with $a \leq 3$ are frequently used in the literature. The partial molar excess property values at infinite dilution, $M_{i}^{\mathrm{E}, \infty}=\lim _{x_{i} \rightarrow 0} M_{i}^{\mathrm{E}}$, are given by

$$
M_{1}^{\mathrm{E}, \infty}=A_{0}+\sum_{m=1}^{a} A_{m}(-1)^{m} \text { and } M_{2}^{\mathrm{E}, \infty}=A_{0}+\sum_{m=1}^{a} A_{m} .
$$

For highly skewed data, using more than four terms may cause spurious oscillations of the $M_{i}^{\mathrm{E}} \mathrm{s}$, and may yield unreliable $M_{i}^{\mathrm{E}, \infty}$ s. Superior flexibility for fitting strongly unsymmetrical curves is provided by Padé approximants [1, 17, 176-178] of order $[a / b]$, where the denominator must never become zero:

$$
\frac{M^{\mathrm{E}}}{x_{1} x_{2}}=\frac{A_{0}+\sum_{m=1}^{a} A_{m}\left(x_{1}-x_{2}\right)^{m}}{1+\sum_{n=1}^{b} B_{n}\left(x_{1}-x_{2}\right)^{n}}, \text { constant } T, P .
$$

As alternatives, expressions based on orthogonal polynomials have been suggested [179-181], e.g., expansions based on Legendre polynomials in $z_{12} \equiv x_{1}-x_{2}$ :

$$
H^{\mathrm{E}}=x_{1} x_{2} \sum_{p=0}^{n_{p}} a_{p} L_{p}\left(z_{12}\right), \text { constant } T, P,
$$

with $L_{0}\left(z_{12}\right)=1, L_{1}\left(z_{12}\right)=z_{12}, L_{2}\left(z_{12}\right)=\left(3 z_{12}^{2}-1\right) / 2, L_{3}\left(z_{12}\right)=\left(5 z_{12}^{3}-3 z_{12}\right) / 2$, and so forth. The summation limit $n_{p}$ is selected as required to fit the available experimental data. If $H^{\mathrm{E}}$ data are available at several temperatures, the temperature dependence of the coefficients $a_{p}$ has to be incorporated via, say,

$$
a_{p}=a_{p 0}+a_{p 1} T+a_{p 2} T^{2}+a_{p 3} T^{3}+\cdots,
$$

or an exponential temperature dependence [182].

Used with necessarily discrete experimental data, Legendre polynomial expansions have the merit that increasing the number of terms to improve the fit will only slightly influence the values of lower-order terms. As pointed out by Pelton and Bale [180, 181], using Legendre expansions in terms of $L_{p}\left(z_{12}\right)$ instead in terms of $L_{p}\left(x_{1}\right)$ has certain advantages. Conversion formulae to calculate Legendre coefficients from Redlich-Kister coefficients (or from power series coefficients) have been given by Pelton and Bale [181], Howald and Eliezer [183], and Tomiska [184].

When the number of components increases to three and beyond, experimental work to determine excess properties increases sharply, thus explaining the scarcity of experimental data for multicomponent mixtures. The situation is aggravated by less reliable empirical/ semiempirical correlating functions describing the composition dependence of multinary 
excess properties, though, for instance, the Redlich-Kister expansion can be generalized without difficulty. However, predictions of multicomponent solution properties from results pertaining to the constituent binaries alone, without ternary (or higher) terms, are always approximate, the most successful correlation of this type being Kohler's equation [185]: it relates the excess molar Gibbs energy $G^{\mathrm{E}, 123}$ of a ternary liquid mixture with mole fractions $\left\{x_{1}, x_{2}, x_{3}\right\}, \sum_{i=1}^{3} x_{i}=1$, to the excess molar Gibbs energies $G^{\mathrm{E}, i j}$ of the three binary subsystems with composition $\left\{x_{i}^{\prime}, x_{j}^{\prime}=1-x_{i}^{\prime}\right\}$, where the mole fractions characterized by a superscript prime are defined by

$$
x_{j}^{\prime} \equiv x_{i} /\left(x_{i}+x_{j}\right) \text { and } x_{j}^{\prime} \equiv x_{j} /\left(x_{i}+x_{j}\right) .
$$

Based on the reasonable approximation that pairwise molecular interactions $i \Leftrightarrow j$ remain constant along lines representing mixtures having a constant composition ratio $x_{i} / x_{j}$, the binary quantities $G^{\mathrm{E}, i j}$ are assumed to depend only on $\left\{x_{i}^{\prime}, x_{j}^{\prime}=1-x_{i}^{\prime}\right\}$, and.

$$
\begin{aligned}
G^{\mathrm{E}, 123}\left(x_{1}, x_{2}, x_{3}\right)= & \left(x_{1}+x_{2}\right)^{2} G^{\mathrm{E}, 12}\left(x_{1}^{\prime}, x_{2}^{\prime}\right) \\
& +\left(x_{1}+x_{3}\right)^{2} G^{\mathrm{E}, 13}\left(x_{1}^{\prime}, x_{3}^{\prime}\right)+\left(x_{2}+x_{3}\right)^{2} G^{\mathrm{E}, 23}\left(x_{2}^{\prime}, x_{3}^{\prime}\right) .
\end{aligned}
$$

Kohler's equation treats the binary subsystems equally, and the model does not impose any restrictions on the functional form of the expressions selected to represent the composition dependence of binary $G^{\mathrm{E}, i j}$ data. Similar comments apply, of course, to $H^{\mathrm{E}, 123}, C_{P}^{\mathrm{E}, 123}$ and $V^{\mathrm{E}, 123}$. Kohler's equation can be generalized to correlate/predict the composition dependence of molar excess properties of multicomponent systems with four or more components. Assuming again that pairwise molecular interactions $i \Leftrightarrow j$ remain constant at conditions imposing a constant composition ratio $x_{i} / x_{j}=x_{i}^{\prime} / x_{j}^{\prime}$, and using Eq. 262 for the binary subsystems with $z_{i j}^{\prime} \equiv x_{i}^{\prime}-x_{j}^{\prime}$, that is,

$$
H^{\mathrm{E}, i j}\left(x_{i}^{\prime}, x_{j}^{\prime}\right)=x_{i}^{\prime} x_{j}^{\prime} \sum_{p=0}^{n_{p}} a_{p} L_{p}\left(z_{i j}^{\prime}\right), \text { constant } T, P,
$$

such a generalized equation for the molar excess enthalpy $H^{\mathrm{E}, 123 \ldots c}$ of a c-component system reads [37]

$$
\left.\begin{array}{rl}
H^{\mathrm{E}, 12 \ldots c}\left(x_{1}, x_{2}, \ldots, x_{c}\right) & \left.=\sum_{j=i+1}^{c} \sum_{i=1}^{c-1}\left[\left(x_{i}+x_{j}\right)^{2} H^{\mathrm{E}, i j}\left(x_{i}^{\prime}, x_{j}^{\prime}\right)\right]\right] \\
& =\sum_{j=i+1}^{c} \sum_{i=1}^{c-1}\left[x_{i} x_{j} \sum_{p=0}^{n_{p}} a_{p} L_{p}\left(z_{i j}^{\prime}\right)\right] .
\end{array}\right\}
$$

For the composition dependence of the excess molar enthalpies $H^{\mathrm{E}, i j}\left(x_{i}^{\prime}, x_{j}^{\prime}\right)$ of the binary subsystems, any function, say, Redlich-Kister, Padé or Legendre polynomial (see Eq. 262), may be used. In fact, each binary system could be represented by a different correlating equation.

Traditionally, the thermodynamic description of real liquid solutions is based on the excess-property formalism presented above. As already pointed out, large numbers of (critically) evaluated experimental data on $G^{\mathrm{E}}$ and $\ln \gamma_{i}^{\mathrm{LR}}, H^{\mathrm{E}}, C_{P}^{\mathrm{E}}$ and $V^{\mathrm{E}}$ of binary liquid 
mixtures are now available in systematic data collections [70, 115-129], and are at the disposal of liquid-state physical chemists and chemical engineers.

For the global thermodynamic description of liquid nonelectrolyte mixtures, $C_{P}^{\mathrm{E}} \mathrm{s}$ are pivotal properties, and taking advantage of the exact relations of Eq. 255, considerable economy in experimental effort can be attained. In order to obtain precise values of $G^{\mathrm{E}}\left(T, P,\left\{x_{i}\right\}\right)$ (as well as of $H^{\mathrm{E}}\left(T, P,\left\{x_{i}\right\}\right)$ and $S^{\mathrm{E}}\left(T, P,\left\{x_{i}\right\}\right)$ ) over wide ranges of temperature at constant pressure $P$ (though well below the vapor-liquid critical region), it suffices to determine $G^{\mathrm{E}}\left(T=T_{\text {ref }}, P,\left\{x_{i}\right\}\right)$ and $H^{\mathrm{E}}\left(T=T_{\text {ref }}, P,\left\{x_{i}\right\}\right)$ at one suitably selected reference temperature (frequently, $T_{\text {ref }}=298.15 \mathrm{~K}$ ), and to measure the temperature dependence of the molar excess heat capacity $C_{P}^{\mathrm{E}}\left(T, P,\left\{x_{i}\right\}\right)$ over a large enough temperature range of interest at constant pressure and at well distributed compositions. These data then permit the consistent determination of the remaining excess properties $G^{\mathrm{E}}, H^{\mathrm{E}}$ and $S^{\mathrm{E}}$ over this temperature range by integration of the relevant differential equations. Compared to the converse approach of determining $G^{\mathrm{E}}$ over a reasonably large temperature range and deriving $H^{\mathrm{E}}, S^{\mathrm{E}}$ and $C_{P}^{\mathrm{E}}$ by differentiation with respect to $T$, it is an obviously advantageous route that will yield more reliable results. This will be especially the case when $G^{\mathrm{E}}$ values are needed at low temperatures where the vapor pressures of the mixtures are small and the conventional determination of $G^{\mathrm{E}}$ is difficult. Well below the vapor-liquid critical region, $C_{P}^{\mathrm{E}}$ of a constant-composition mixture frequently shows a simple temperature dependence, that is, at constant pressure it can be approximated satisfactorily by [157],

$$
C_{P}^{\mathrm{E}} / R=a_{3}+a_{4} \tau+a_{5} \tau^{2},
$$

where $\tau \equiv T_{\text {ref }} / T$. Using the differential equations presented in Eq. 255, integration over temperature at constant pressure and constant composition yields

$$
\begin{aligned}
& H^{\mathrm{E}} / R T=a_{3}+\left(a_{2}-a_{3}+a_{5}\right) \tau-a_{5} \tau^{2}-a_{4} \tau \ln \tau, \\
& S^{\mathrm{E}} / R=a_{1}+a_{4}+a_{5} / 2-a_{4} \tau-a_{5} \tau^{2} / 2-a_{3} \ln \tau,
\end{aligned}
$$

and

$$
G^{E} / R T=-a_{1}+a_{3}-a_{4}-a_{5} / 2+\left(a_{2}-a_{3}+a_{4}+a_{5}\right) \tau-a_{5} \tau^{2} / 2+a_{3} \ln \tau-a_{4} \tau \ln \tau .
$$

The dimensionless coefficients $a_{i}$ depend, of course, on pressure and composition; they are related to the corresponding molar excess quantities at $\left\{T=T_{\text {ref }}, P,\left\{x_{i}\right\}\right\}$ as follows: $C_{P}^{\mathrm{E}}\left(T_{\text {ref }}\right) / R=a_{3}+a_{4}+a_{5}, \quad H^{\mathrm{E}}\left(T_{\text {ref }}\right) / R T_{\text {ref }}=a_{2}, \quad S^{\mathrm{E}}\left(T_{\text {ref }}\right) / R=a_{1}, \quad$ and $G^{\mathrm{E}}\left(T_{\text {ref }}\right) / R T_{\text {ref }}=-a_{1}+a_{2}$. Analogous expressions may be derived if the temperature dependence of $C_{P}^{\mathrm{E}}$, at constant pressure and composition, is described by a polynomial in $T$ instead of $T^{-1}$. Global studies of this kind are, however, quite rare, with some of the most careful investigations being those of Ziegler and colleagues [186, 187].

Classical thermodynamics does not constrain the signs of particular excess properties, that is, $G^{E}, H^{\mathrm{E}}$ and $S^{\mathrm{E}}$ can each be positive or negative, and quite a few systems are known where the sign changes with composition (sigmoidal composition dependence: one maximum, one minimum). Very rarely so-called $M$-shaped excess enthalpies have been reported: for the binary liquid mixture \{benzonitrile + benzene \}, at $298.15 \mathrm{~K}, 308.15 \mathrm{~K}$, and $318.15 \mathrm{~K}$ and ambient pressure, $H^{\mathrm{E}}$ changes its sign with composition two times, that is, it exhibits two maxima and one minimum [188]. Thermodynamics restricts, however, the admissible combinations of signs for these three excess properties: Eq. 249 clearly 
Fig. $1 W$-shaped molar excess heat capacities $C_{P}^{\mathrm{E}}$ at constant pressure of three binary liquid mixtures of type $\{1,4-$ dioxane + n-alkane $\}$, i.e. $\left\{x_{1} 1,4-\right.$ $\left.\mathrm{C}_{4} \mathrm{H}_{8} \mathrm{O}_{2}+\left(1-x_{1}\right) \mathrm{C}_{n} \mathrm{H}_{2 n+2}\right\}$, $n=7,10$ and 14 , at $298.15 \mathrm{~K}$ and ambient pressure. The circles and triangles represent our experimental results [189]. The figure was reproduced from $\mathrm{E}$. Wilhelm, J. Solution Chem. 43, 525-576 (2014) [157]

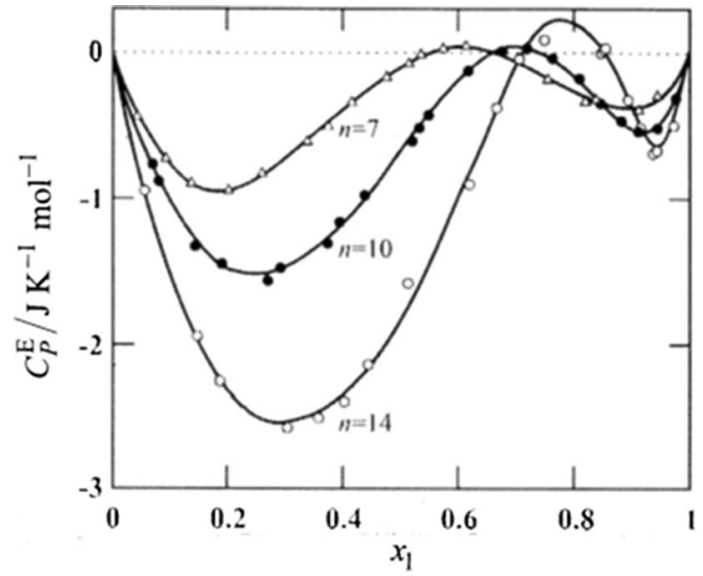

shows that a positive $G^{\mathrm{E}}$ with a negative $H^{\mathrm{E}}$ and a positive $S^{\mathrm{E}}$ is not possible, nor a negative $G^{E}$ with a positive $H^{\mathrm{E}}$ and a negative $S^{\mathrm{E}}$. In passing I note that our research group in France was the first to report (in 1982) $W$-shaped excess heat capacities $C_{P}^{\mathrm{E}}$ vs. $x_{1}$ for $\{1,4$-dioxane $\left.+\mathrm{n}-\mathrm{C}_{n} \mathrm{H}_{2 n+2}\right\}$ at $298.15 \mathrm{~K}, n=7,10,14$ [189]: $C_{P}^{\mathrm{E}}$ exhibits two minima and one maximum, see Fig. 1.

\subsection{Excess Molar Gibbs Energy and Lewis-Randall Activity Coefficients (in Particular at Infinite Dilution)}

Activity coefficients $\gamma_{i}^{\mathrm{LR}, \infty}$ at infinite dilution characterize the thermodynamic behavior of a single solute molecule completely surrounded by solvent molecules, hence it usually indicates maximum non-ideality and-in the absence of $i \Leftrightarrow i$ interactions-it provides important information on solute-solvent interactions $i \Leftrightarrow j$. Thus, they are of great value in chemical engineering and are key parameters in the discussion of dilute solutions encountered in environmental studies [190-192]. In fact, given the infinite-dilution activity coefficients of each component in the other in a binary mixture, values of parameters in popular two-parameter activity coefficient models can be easily obtained; in turn, these can be used for phase equilibrium predictions over the entire composition range [193]. However, prediction quality depends strongly on the model used, and whether the mixture is only slightly or strongly nonideal. With improved experimental techniques $[170,171,194]$, precise measurements at low concentrations can be made with less effort compared to conventional VLE measurements, and with greater accuracy since extrapolation of activity coefficients obtained at higher mole fractions to infinite dilution is quite demanding.

For the prediction of the composition dependence of LR-based $G^{\mathrm{E}}$, many empirical equations have been proposed, and for binary mixtures, some of the simpler ones are special cases of one of the following power series expansions in the mole fractions:

$$
\frac{G^{\mathrm{E}}}{x_{1} x_{2} R T}=B^{\prime}+C^{\prime}\left(x_{1}-x_{2}\right)+D^{\prime}\left(x_{1}-x_{2}\right)^{2}+E^{\prime}\left(x_{1}-x_{2}\right)^{3}+\cdots,
$$

see Eq. 259 (Redlich-Kister expansion), or 


$$
\frac{x_{1} x_{2} R T}{G^{\mathrm{E}}}=B+C\left(x_{1}-x_{2}\right)+D\left(x_{1}-x_{2}\right)^{2}+E\left(x_{1}-x_{2}\right)^{3}+\cdots .
$$

With restriction to two parameters and on rearrangement, i.e., $B^{\prime}+C^{\prime}=A_{21}^{\prime}$ and $B^{\prime}-C^{\prime}=A_{12}^{\prime}$, Eq. 272 yields the equivalent (two-parameter) 3-suffix Margules ${ }^{3}$ equation

$$
\frac{G^{\mathrm{E}}}{x_{1} x_{2} R T}=A_{21}^{\prime} x_{1}+A_{12}^{\prime} x_{2},
$$

with the following expressions for the LR activity coefficients:

$$
\begin{aligned}
& \frac{G_{1}^{\mathrm{E}}}{R T}=\ln \gamma_{1}^{\mathrm{LR}}=x_{2}^{2}\left[A_{12}^{\prime}+2 x_{1}\left(A_{21}^{\prime}-A_{12}^{\prime}\right)\right], \text { and } \ln \gamma_{1}^{\mathrm{LR}, \infty}=A_{12}^{\prime}=B^{\prime}-C^{\prime}, \\
& \frac{G_{2}^{\mathrm{E}}}{R T}=\ln \gamma_{2}^{\mathrm{LR}}=x_{1}^{2}\left[A_{21}^{\prime}+2 x_{2}\left(A_{12}^{\prime}-A_{21}^{\prime}\right)\right], \text { and } \ln \gamma_{2}^{\mathrm{LR}, \infty}=A_{21}^{\prime}=B^{\prime}+C^{\prime} .
\end{aligned}
$$

With restriction to two parameters and on rearrangement, i.e., $1 /(B-C)=A_{12}$, and $1 /(B+C)=A_{21}$, Eq. 273 yields the equivalent two-parameter van Laar equation [188, 189]

$$
\frac{G^{\mathrm{E}}}{x_{1} x_{2} R T}=\frac{A_{12} A_{21}}{A_{12} x_{1}+A_{21} x_{2}},
$$

with the following expressions for the LR activity coefficients:

$$
\begin{aligned}
& \frac{G_{1}^{\mathrm{E}}}{R T}=\ln \gamma_{1}^{\mathrm{LR}}=A_{12}\left(1+\frac{A_{12}}{A_{21}} \frac{x_{1}}{x_{2}}\right)^{-2}, \text { and } \ln \gamma_{1}^{\mathrm{LR}, \infty}=A_{12}=\frac{1}{B-C}, \\
& \frac{G_{2}^{\mathrm{E}}}{R T}=\ln \gamma_{2}^{\mathrm{LR}}=A_{21}\left(1+\frac{A_{21}}{A_{12}} \frac{x_{2}}{x_{1}}\right)^{-2} \text {, and } \ln \gamma_{2}^{\mathrm{LR}, \infty}=A_{21}=\frac{1}{B+C} .
\end{aligned}
$$

\footnotetext{
${ }^{3}$ Max Margules was an Austrian physicist. Born in Brody, Galicia, a former crownland of the AustroHungarian Empire, on April 23, 1856, he started his studies in mathematics, physics and chemistry at the University of Wien (Vienna), Austria, in 1872. Among his teachers were Ludwig Boltzmann and Josef Loschmidt. After a few years as Assistant at the Central Institute of Meteorology in Wien, he went to Berlin for additional studies in mathematical physics (1879/1880) and was habilitated at the University of Wien after his return. However, in 1882 Margules resigned from this academic position and rejoined the Central Institute of Meteorology. During the first years at this institution, he continued to pursue physical and physical-chemical research parallel to his work on theoretical meteorology. In 1906 he voluntarily retired at the age of fifty on a very modest pension. This was also the year of his last meteorological publication, dedicated to the theory of storms:

Margules, M.: Zur Sturmtheorie. Meteorolog. Z. 23, 481-497 (1906).

His small pension and the inflation after the end of World War I led to a life in poverty, which was compounded by the general state of malnutrition of the Austrian population and his refusal to accept help from colleagues and/or the Austrian Meteorological Society (he was awarded the Hann Medal in 1919 but declined the associated honorarium). Max Margules died of starvation in Perchtoldsdorf near Wien (Vienna), Austria, on October 4, 1920. He contributed significantly and lastingly to meteorology and thermodynamics [195]. Obituaries were prepared by F. M. Exner (Meteorolog. Z. 37, 322-324 (1920)) and E. Gold (Nature 106, 286-287 (1920)).
} 
If for a binary system precise and plentiful data are available, the use of a 3-parameter Redlich-Kister equation (see Eqs. 259 or 272) is frequently indicated, i.e.,

$$
\frac{G^{\mathrm{E}}}{x_{1} x_{2} R T}=A^{\prime}+B^{\prime}\left(x_{1}-x_{2}\right)+C^{\prime}\left(x_{1}-x_{2}\right)^{2},
$$

with the following expressions for the LR activity coefficients:

$$
\begin{aligned}
& \ln \gamma_{1}^{\mathrm{LR}}=x_{2}^{2}\left[A^{\prime}+3 B^{\prime}+5 C^{\prime}-\left(4 B^{\prime}+16 C^{\prime}\right) x_{2}+12 C^{\prime} x_{2}^{2}\right], \text { and } \ln \gamma_{1}^{\mathrm{LR}, \infty}=A^{\prime}-B^{\prime}+C^{\prime}, \\
& \ln \gamma_{2}^{\mathrm{LR}}=x_{1}^{2}\left[A^{\prime}-3 B^{\prime}+5 C^{\prime}+\left(4 B^{\prime}-16 C^{\prime}\right) x_{2}+12 C^{\prime} x_{2}^{2}\right], \text { and } \ln \gamma_{2}^{\mathrm{LR}, \infty}=A^{\prime}+B^{\prime}+C^{\prime} .
\end{aligned}
$$

Combining the results for $\ln \gamma_{1}^{\mathrm{LR}, \infty}$ and $\ln \gamma_{2}^{\mathrm{LR}, \infty}$ with the value of $G^{\mathrm{E}} / R T$ at $x_{1}=x_{2}=0.5$ yields.

$$
\begin{aligned}
& A^{\prime}=4 G^{\mathrm{E}}\left(x_{1}=0.5\right) / R T, B^{\prime}=\left(\ln \gamma_{2}^{\mathrm{LR}, \infty}-\ln \gamma_{1}^{\mathrm{LR}, \infty}\right) / 2, \\
& C^{\prime}=\left(\ln \gamma_{2}^{\mathrm{LR}, \infty}+\ln \gamma_{1}^{\mathrm{LR}, \infty}\right) / 2-A^{\prime} .
\end{aligned}
$$

Note that the Redlich-Kister equation, Eq. 280, may also be transformed into an alternative, equivalent polynomial known as the 4-suffix Margules equation [1, 17]

$$
\frac{G^{\mathrm{E}}}{x_{1} x_{2} R T}=A_{21}^{\prime} x_{1}+A_{12}^{\prime} x_{2}-C^{\prime} x_{1} x_{2} .
$$

In 1964 Wilson suggested a novel equation for $G^{\mathrm{E}}$ by introducing the local mole fraction of component $i$ in a mixture $\{i+j\}$ [198], a concept which has been developed impressively since then [137, 199-209]. For a binary mixture, the molar excess Gibbs energy is given by

$$
\begin{aligned}
\frac{G^{\mathrm{E}}}{R T} & =-x_{1} \ln \left(x_{1}+x_{2} \Lambda_{12}\right) \\
& -x_{2} \ln \left(x_{2}+x_{1} \Lambda_{21}\right)
\end{aligned}
$$

and the activity coefficients are

$$
\begin{gathered}
\ln \gamma_{1}^{\mathrm{LR}}=-\ln \left(x_{1}+x_{2} \Lambda_{12}\right)+x_{2} \Gamma, \\
\ln \gamma_{2}^{\mathrm{LR}}=-\ln \left(x_{2}+x_{1} \Lambda_{21}\right)-x_{1} \Gamma, \\
\Gamma=\frac{\Lambda_{12}}{x_{1}+x_{2} \Lambda_{12}}-\frac{\Lambda_{21}}{x_{2}+x_{1} \Lambda_{21}} .
\end{gathered}
$$

Thus, at infinite dilution we obtain

$$
\ln \gamma_{1}^{\mathrm{LR}, \infty}=-\ln \Lambda_{12}+1-\Lambda_{21} \text { and } \ln \gamma_{2}^{\mathrm{LR}, \infty}=-\ln \Lambda_{21}+1-\Lambda_{12} .
$$

An iterative procedure is required to evaluate the adjustable parameters $\Lambda_{12}$ and $\Lambda_{21}$. In Wilson's derivation, they are related to the pure-component molar volumes and to characteristic interaction energy differences, i.e., 


$$
\Lambda_{i j}=\frac{V_{i}^{\mathrm{L}, *}}{V_{j}^{\mathrm{L}, *}} \exp \left(-\frac{\lambda_{i j}-\lambda_{i i}}{R T}\right) .
$$

Numerical values of the parameters $\lambda_{i j}-\lambda_{i i}$ can only be found through reduction of experimental VLE data. The Wilson equation is a very flexible equation with a built-in temperature dependence; it is able to represent mixtures exhibiting strong deviations from ideality and is easily generalized to describe multicomponent behavior using only binary parameters. However, Wilson's equation is unable to predict limited miscibility, and should therefore be used only for liquid systems where the components are completely miscible.

Enormous research efforts have been invested in developing the local composition concept, for instance in developing the NRTL equation, the UNIQUAC and UNIFAC formalism, and the DISQUAC model [137, 199-209]. This topic definitely deserves a review of its own.

\subsection{Henry's Law: An Alternative Ideal-Solution Model for Liquid Systems}

In Sect. 2.4, I presented an ideal-solution model derived from the Lewis-Randall rule, that serves as the basis of the excess property formalism. This approach for the discussion of liquid solution properties is entirely appropriate as long as the constituent components are stable as pure liquids at $T$ and $P$ of the solution. However, solutions of gases in liquids pose a problem, since the dissolved gas does not exist in the pure state as a liquid at $T$ and $P$ of the solution, thereby precluding any measurements of its properties in this state. In addition, the solubility of gases in liquids is usually quite small, hence experimental data are usually collected only over a rather small part of the composition range $[70,115,116$, 210-215].

Consider a binary system of solute 2 dissolved in solvent 1 . A general criterion for phase equilibrium at $T$ and $P$ is the equality of the fugacity $f_{i}^{\pi}$ of each one of the components in the coexisting phases. Thus, for the specific case of VLE, where $\pi=\mathrm{V}$ or $\mathrm{L}$,

$$
f_{i}^{\mathrm{V}}\left(T, P, x_{i}^{\mathrm{V}}\right)=f_{i}^{\mathrm{L}}\left(T, P, x_{i}^{\mathrm{L}}\right), i=1 \text { or } 2 .
$$

The link with experiment may be formally established by expressing the component fugacities in the vapor phase in terms of the respective fugacity coefficients $\phi_{i}^{\mathrm{V}}$,

$$
f_{i}^{\mathrm{V}}\left(T, P, x_{i}^{\mathrm{V}}\right)=\phi_{i}^{\mathrm{V}}\left(T, P, x_{i}^{\mathrm{V}}\right) x_{i}^{\mathrm{V}} P, i=1 \text { or } 2 \text {, valid for } 0 \leq x_{i}^{\mathrm{V}} \leq 1,
$$

while the liquid-phase fugacities of the components are expressed with the help of appropriately normalized liquid-phase activity coefficients. Two entirely equivalent approaches are in use: the symmetric convention is based on the Lewis-Randall rule, Eq. 196, and leads to (i.e., for both solvent and solute)

$$
f_{i}^{\mathrm{L}}\left(T, P, x_{i}^{\mathrm{L}}\right)=\gamma_{i}^{\mathrm{LR}}\left(T, P, x_{i}^{\mathrm{L}}\right) x_{i}^{\mathrm{L}} f_{i}^{\mathrm{L}, *}(T, P), i=1 \text { or } 2, \text { valid for } 0 \leq x_{i}^{\mathrm{L}} \leq 1,
$$

where

$$
f_{i}^{\mathrm{L}, *}(T, P)=P \phi_{i}^{\mathrm{L}, *}(T . P)
$$

denotes the fugacity of pure component $i$ in either a real or a hypothetical liquid state at $T$ and $P$ of the liquid solution, and $\phi_{i}^{\mathrm{L}, *}(T . P)$ is the corresponding pure-substance fugacity 
coefficient (see Eqs. 131-133). As I have already indicated, $\gamma_{i}^{\mathrm{LR}}$ is predominantly used for the thermodynamic description of liquid mixtures when all components are considered stable as pure liquids at $T$ and $P$ of interest, and

$$
\gamma_{i}^{\mathrm{LR}} \rightarrow 1 \text { as } x_{i}^{\mathrm{L}} \rightarrow 1, \text { constant } T, P,
$$

applies to all components of the solution: the activity coefficients are said to be normalized symmetrically. At all other compositions $x_{i}^{\mathrm{L}} \neq 1$, deviations of $\gamma_{i}^{\mathrm{LR}}$ from unity measure deviations of real solution behavior from ideal LR behavior (ratio measure). This $(\phi, \gamma)$ -approach is also known, somewhat imprecisely, as being based on Raoult's law.

Alternatively, ideal-solution behavior may be based on Henry's law (HL), that is, the validity of the linear relation

$$
f_{i}^{\mathrm{id}}\left(T, P,\left\{x_{i}\right\}\right) \equiv f_{i}^{\mathrm{HL}}\left(T, P, x_{i}^{\mathrm{L}}\right)=x_{i}^{\mathrm{L}} h_{i, j}(T, P), i=1 \text { or } 2,
$$

over the entire composition range $0 \leq x_{i}^{\mathrm{L}} \leq 1$ is assumed, where $h_{i, j}(T, P)$ denotes the Henry fugacity (also known as Henry's law constant) as defined below by Eq. 300a. Traditionally, for compact notation, no superscript L is attached to the Henry fugacity. The corresponding activity coefficient $\gamma_{i}^{\mathrm{HL}}$ measures how much the component fugacity of $i$ in the real solution differs from the component fugacity of $i$ corresponding to the HL-idealization Eq. 296 (ratio measure) [17, 66, 169]. Thus,

$$
f_{i}^{\mathrm{L}}\left(T, P, x_{i}^{\mathrm{L}}\right)=\gamma_{i}^{\mathrm{HL}}\left(T, P, x_{i}^{\mathrm{L}}\right) x_{i}^{\mathrm{L}} h_{i, j}(T, P) .
$$

The value of the Henry fugacity depends on $T$ and $P$ and also on the identity of the solvent (the other component), hence the double subscript $i, j$ has been added; and $h_{i, j}(T, P)$ has to be determined for each binary system. HL-based activity coefficients are predominantly used for the thermodynamic description of dilute liquid solutions and, in particular, if the experimental temperature is above the critical temperature of one component of the solution (gas solubility). From Eqs. 297 and 300a (presented below), it follows that

$$
\gamma_{i}^{\mathrm{HL}} \rightarrow 1 \text { as } x_{i}^{\mathrm{L}} \rightarrow 0, \text { constant } T, P .
$$

That is, at nonzero compositions, $x_{i}^{\mathrm{L}} \neq 0$, deviations of $\gamma_{i}^{\mathrm{HL}}$ from unity quantify real solution behavior vis-à-vis ideal HL behavior.

The activity coefficients are said to be normalized unsymmetrically, when for the solvent $(i=1)$ the LR convention Eq. 295 applies, and for the (frequently supercritical) solute $(i=2)$ the HL convention Eq. 298 is used. The limiting behavior of the activity coefficients characterizing such a binary liquid solution is thus given by

$$
\left.\begin{array}{l}
\gamma_{1}^{\mathrm{LR}} \rightarrow 1 \text { as } x_{1}^{\mathrm{L}} \rightarrow 1, \\
\gamma_{2}^{\mathrm{HL}} \rightarrow 1 \text { as } x_{2}^{\mathrm{L}} \rightarrow 0
\end{array}\right\}, \text { constant } T, P .
$$

For obvious reasons, this approach is also called a $(\phi, \gamma)$-method. For details, see Refs. 1, 17, 66, 169 and 216-219.

Figure 2 presents schematically the composition dependence of the component fugacity $f_{2}\left(T, P, x_{2}\right)$, at constant temperature and pressure, in a binary liquid solution (solid curve) exhibiting a positive deviation from the Lewis-Randall rule, Eq. 196. $f_{2}^{*}(T, P)$ is the fugacity of pure liquid component 2 , and $h_{2,1}(T, P)$ is the Henry fugacity (also known as Henry's law constant) of solute 2 dissolved in solvent 1 . For the sake of simplicity, the 


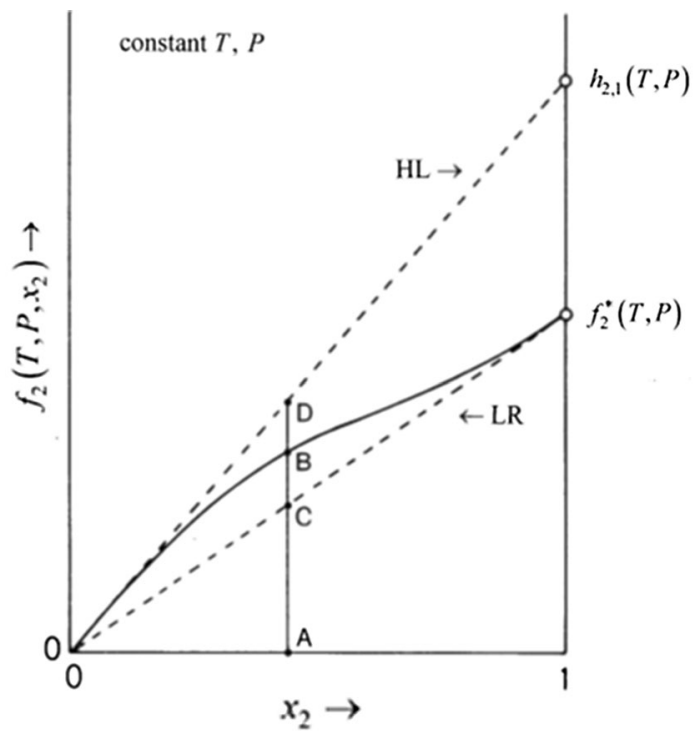

Fig. 2 Composition dependence (schematic) of the component fugacity $f_{2}\left(T, P, x_{2}\right)$ in a binary liquid solution (solid curve) at constant $T$ and $P$ showing a positive deviation from the Lewis-Randall rule, Eq. 196. $f_{2}^{*}(T, P)$ is the fugacity of pure liquid component $2, h_{2,1}(T, P)$ is the Henry fugacity (also known as Henry's law constant) of solute 2 dissolved in solvent 1 , and $x_{2}=1-x_{1}$ is the mole fraction of component 2 in the solution. For compact notation, the superscripts L have been omitted. The dashed lines represent two popular ideal-solution models: the one based on the Lewis-Randall rule, i.e. Equation 196, is labeled LR, and the one based on Henry's law, i.e. Equation 296, is labeled HL The corresponding activity coefficients that are used to quantitatively characterize reality in a binary solution are defined by Eqs. 293 (LR) and 297 (HL), respectively. For the solute $i=2$, the activity coefficients (at any selected composition $x_{2}$ ), are thus given geometrically by $\gamma_{2}^{\mathrm{LR}}\left(T, P, x_{2}\right)=f_{2}\left(T, P, x_{2}\right) / f_{2}^{\mathrm{LR}}\left(T, P, x_{2}\right)=f_{2}\left(T, P, x_{2}\right) / x_{2} f_{2}^{*}\left(T, P, x_{2}\right)=\overleftrightarrow{\mathrm{AB}} / \overleftrightarrow{\mathrm{AC}}$, or $\gamma_{2}^{\mathrm{HL}}\left(T, P, x_{2}\right)=f_{2}\left(T, P, x_{2}\right) / f_{2}^{\mathrm{HL}}\left(T, P, x_{2}\right)=f_{2}\left(T, P, x_{2}\right) / x_{2} h_{2,1}\left(T, P, x_{2}\right)=\overleftrightarrow{\mathrm{AB}} / \overleftrightarrow{\mathrm{AD}}$. Because of the intercept theorem, their ratio is independent of the composition (see also Eq. 307): $\gamma_{2}^{\mathrm{LR}}\left(T, P, x_{2}\right) / \gamma_{2}^{\mathrm{HL}}\left(T, P, x_{2}\right)=\overleftrightarrow{\mathrm{AD}} / \overleftrightarrow{\mathrm{AC}}=h_{2,1}(T, P) / f_{2}^{*}(T, P)$. Evidently, for positive deviations from LRideality (which are more common than negative deviations) $\gamma_{i}^{\mathrm{LR}}>1$ and $\gamma_{i}^{\mathrm{HL}}<1$, and conversely, for negative deviations from LR-ideality $\gamma_{i}^{\mathrm{LR}}<1$ and $\gamma_{i}^{\mathrm{HL}}>1$. However, when the focus is on solutions of a supercritical solute 2 (a gas) in a liquid solvent 1, the pure solute does not exist as a liquid at the experimental conditions. In addition, the solubility of such a gas is limited at given $T$ and $P$, hence experimental results provide the composition dependence of $f_{2}\left(T, P, x_{2}\right)$ for only a part of the composition range, i.e. for $0 \leq x_{2}<1$. In this case, the unsymmetric convention is usually selected: it has the advantage that Henry fugacities and hence activity coefficients $\gamma_{2}^{\mathrm{HL}}\left(T, P, x_{2}\right)$ are unambiguously accessible via an experimental procedure (cf. Equation 301) [17, 66, 169, 216, 219]. The figure was reproduced from E. Wilhelm, J. Solution Chem. 44, 1004-1061 (2015) [66]

superscripts L have been omitted. The dashed lines represent the two most popular idealsolution models discussed in this review: the one labeled LR corresponds to Eq. 196, and the other, labeled HL, corresponds to Eq. 296. As shown below via Eq. 300a, the HL line becomes a tangent to $f_{2}\left(T, P, x_{2}\right)$ in the limit $x_{2} \rightarrow 0$, while according to Eq. $300 \mathrm{~b}$, the LR line becomes a tangent to $f_{2}\left(T, P, x_{2}\right)$ in the limit $x_{2} \rightarrow 1$.

Since the limiting value of the ratio $f_{i}^{\pi} / x_{i}^{\pi}$ for $x_{i}^{\pi} \rightarrow 0$ at constant $T$ and $P$ is known from experiment to be finite, $f_{i}^{\pi}$ must also become zero at $x_{i}^{\pi}=0$, and by de l'Hôpital's rule 


$$
\lim _{x_{i}^{\pi} \rightarrow 0}\left(\frac{f_{i}^{\pi}}{x_{i}^{\pi}}\right)=\left(\frac{\mathrm{d} f_{i}^{\pi}}{\mathrm{d} x_{i}^{\pi}}\right)_{x_{i}^{\pi}=0}=h_{i, j}^{\pi}(T, P), \text { constant } T, P,
$$

is obtained. Equation 300a summarizes Henry's law; it defines the Henry fugacity $h_{i, j}^{\pi}(T, P)$ of component $i$ dissolved in component $j$ for any phase $\pi(\mathrm{L}$ or $\mathrm{V})$ and identifies the limiting slope of the curve $f_{i}^{\pi}$ vs. $x_{i}^{\pi}$ at constant $T$ and $P$ as $h_{i, j}^{\pi}(T, P)$. Henry's law is a limiting law, and for real solutions it is approximately valid for small values of $x_{i}^{\pi}$, with the experimental precision determining the observed apparent validity range [17, 66, 169, 216, 219].

As shown in Refs. 17, 66, 169 and 219, at the other end of the composition range, that is, for $x_{i}^{\pi} \rightarrow 1$, at constant temperature and pressure we obtain

$$
\lim _{x_{i}^{\pi} \rightarrow 1}\left(\frac{f_{i}^{\pi}}{x_{i}^{\pi}}\right)=\left(\frac{\mathrm{d} f_{i}^{\pi}}{\mathrm{d} x_{i}^{\pi}}\right)_{x_{i}^{\pi}=1}=f_{i}^{\pi, *}(T, P), \text { constant } T, P,
$$

where $f_{i}^{\pi, *}(T, P)$ denotes the fugacity of pure component $i$ at $T$ and $P$ of the solution and in the same physical state. Equation 300b summarizes the Lewis-Randall rule; it is valid in any phase $\pi\left(\mathrm{L}\right.$ or $\mathrm{V}$ ) and shows that in the limit $x_{i}^{\pi} \rightarrow 1$ both $f_{i}^{\pi}$ and the limiting slope of the curve $f_{i}^{\pi}$ vs. $x_{i}^{\pi}$ at constant $T$ and $P$ become equal to the fugacity of pure $i$ in phase $\pi$. The Lewis-Randall rule is a limiting law, and for real solutions it is approximately valid for values of $x_{i}^{\pi}$ near unity, with the experimental precision determining the observed apparent validity range [17, 66, 169, 216, 219].

Focusing now on gas solubilities, the Henry fugacity of supercritical solute 2 dissolved in liquid solvent 1 is defined by Eq. 300a, with $\pi=\mathrm{L}$. Because of vapor-liquid phase equilibrium, see Eq. 291, in conjunction with Eqs. 292, 297 and 298, and adopting the widely used notation $x_{i}^{\mathrm{V}} \equiv y_{i}$ and $x_{i}^{\mathrm{L}} \equiv x_{i}$, we arrive at the classical experimental prescription for the determination of $h_{2,1}\left(T, P_{\sigma, 1}\right)$ from isothermal VLE experiments (measurement of $P$, $x_{2}$ and $\left.y_{2}\right)$ at decreasing total pressures $P \rightarrow P_{\sigma, 1}(T)$ and concomitantly decreasing $x_{2} \rightarrow 0$ and $y_{2} P \rightarrow 0$ :

$$
h_{2,1}\left(T, P_{\sigma, 1}\right)=\lim _{x_{2} \rightarrow 0}\left(\frac{f_{2}^{\mathrm{L}}\left(T, P, x_{2}\right)}{x_{2}}\right)=\lim _{\substack{x_{2} \rightarrow 0 \\ y_{2} P \rightarrow 0}}\left(\frac{\phi_{2}^{\mathrm{V}}\left(T, P, y_{2}\right) y_{2} P}{x_{2}}\right), \text { constant } T .
$$

That is to say, the Henry fugacity referring to solute 2 dissolved in the liquid phase (consisting essentially of solvent 1 ) is obtained as the intercept of a plot, at constant $T$, of $\phi_{2}^{\mathrm{V}}\left(T, P, y_{2}\right) y_{2} P / x_{2}$ against $x_{2}$ or $y_{2} P$. Entirely equivalent expressions relating the Henry fugacity to limiting slopes (see Eq. 300a) may be derived. When applying Eq. 301 to experimental data, the vapor-phase fugacity coefficient $\phi_{2}^{\mathrm{V}}\left(T, P, y_{2}\right)$ must be calculated with a suitable vapor-phase EOS. Since the majority of gas-solubility measurements is performed in the low to moderate pressure domain, the virial equation of state is the equation of choice: it has a sound theoretical basis, and it is superior to cubic equations of state. The computational convenience associated with a volume-explicit version leads to the excellent, widely used approximation

$$
Z^{\mathrm{V}}\left(T, P,\left\{y_{i}\right\}\right)=1+B\left(T,\left\{y_{i}\right\}\right) P / R T,
$$

where $B\left(T,\left\{y_{i}\right\}\right)$ is the second mixture virial coefficient [49, 220-223] (note that $B$ refers to the virial equation in amount density and is usually the quantity listed in data 
compilations). In turn, for the calculation of the fugacity coefficient of the gas in the binary vapor mixture Eq. 153a is used, yielding (see Eq. 153b)

$$
\ln \phi_{2}^{\mathrm{V}}=\frac{P}{R T}\left(B_{22}+y_{1}^{2} \delta_{12}\right),
$$

where $\delta_{12}$ is defined by Eq. 248 .

The temperature dependence of the Henry fugacity is given by [17, 66, 169, 216, 219]

$$
\left(\frac{\partial \ln h_{2,1}(T, P)}{\partial T}\right)_{P}=-\frac{H_{2}^{\mathrm{L}, \infty}-H_{2}^{\mathrm{pg}, *}}{R T^{2}}=-\frac{\Delta H_{2}^{\infty}(T, P)}{R T^{2}},
$$

and its pressure dependence is given by $[17,66,169,216,219]$

$$
\left(\frac{\partial \ln h_{2,1}(T, P)}{\partial P}\right)_{T}=\frac{V_{2}^{\mathrm{L}, \infty}}{R T} .
$$

Here, $H_{2}^{\mathrm{L}, \infty}$ is the partial molar enthalpy of solute 2 at infinite dilution in liquid solvent $1, H_{2}^{\mathrm{pg}, *}$ denotes the molar enthalpy of the pure solute in the perfect-gas state, and $\Delta H_{2}^{\infty}(T, P)$ is the partial molar enthalpy change on solution (this notation is generally preferred over $H_{2}^{\mathrm{R}, \mathrm{L}, \infty}$, the partial molar residual enthalpy at infinite dilution [66, 169]). $V_{2}^{\mathrm{L}, \infty}$ is the partial molar volume of the solute at infinite dilution in the liquid solvent. Equation 304 provides the basis for obtaining partial molar enthalpy changes on solution via van't Hoff analysis of high-precision solubility data of gases in liquids (see below).

For the reduction, correlation, and further use of high-precision gas solubility data, as suggested by Eq. 301, it is advantageous to select at each temperature the vapor pressure $P_{\sigma, 1}(T)$ of the solvent as reference pressure. With this convention, the Henry fugacity of solute 2 dissolved in solvent 1 at any other pressure is obtained via integration of Eq. 305 :

$$
h_{2,1}(T, P)=h_{2,1}\left(T, P_{\sigma, 1}(T)\right) \exp \left[\int_{P_{\sigma, 1}}^{P} \frac{V_{2}^{\mathrm{L}, \infty}(T, P)}{R T} \mathrm{~d} P\right] .
$$

The exponential in Eq. 306 is known as Poynting correction factor; its evaluation requires reliable data on the partial molar volume of the solute at infinite dilution [224-236].

Since the approaches to the thermodynamic description of VLE introduced above are equivalent, various key quantities associated with them are, of course, connected with each other by exact relations [66, 169, 216-219, 237-239]. For instance, focusing on binary liquid mixtures, comparison of Eq. 293 with Eq. 297 yields $(i=1$ or $2, j=1$ or $2, i \neq j$ )

$$
\frac{\gamma_{i}^{\mathrm{LR}}\left(T, P, x_{i}\right)}{\gamma_{i}^{\mathrm{HL}}\left(T, P, x_{i}\right)}=\frac{h_{i, j}(T, P)}{f_{i}^{\mathrm{L}, *}(T, P)},
$$

independent of composition. Thus, for the LR activity coefficient at infinite dilution one obtains

$$
\gamma_{i}^{\mathrm{LR}, \infty}=\lim _{x_{i} \rightarrow 0} \gamma_{i}^{\mathrm{LR}}=h_{i, j} / f_{i}^{\mathrm{L}, *}
$$


and hence

$$
\begin{gathered}
\gamma_{i}^{\mathrm{HL}}=\gamma_{i}^{\mathrm{LR}} / \gamma_{i}^{\mathrm{LR}, \infty}, \\
\lim _{x_{i} \rightarrow 1} \gamma_{i}^{\mathrm{HL}}=1 / \gamma_{i}^{\mathrm{LR}, \infty} .
\end{gathered}
$$

For compact notation, the specifications $\left(T, P, x_{i}\right)$, etc. have been omitted.

From the definitions Eqs. 129 and 300a follows the important, generally valid relation $[66,169]$, here specified to apply to the liquid solution phase (that is, $\pi=\mathrm{L}$ ):

$$
\left.\begin{array}{l}
\phi_{i}^{\mathrm{L}, \infty}(T, P)=\lim _{x_{i} \rightarrow 0} \phi_{i}^{\mathrm{L}}\left(T, P, x_{i}\right)=\frac{1}{P} \lim _{x_{i} \rightarrow 0}\left[\frac{f_{i}^{\mathrm{L}}\left(T, P, x_{i}\right)}{x_{i}}\right]=\frac{h_{i, j}(T, P)}{P}, \text { constant } T, P, \\
\phi_{i}^{\mathrm{L}, \infty}(T, P)=\exp \left(\frac{G_{i}^{\mathrm{R}, \mathrm{L}, \infty}(T, P)}{R T}\right)=\exp \left(\frac{\mu_{i}^{\mathrm{R}, \mathrm{L}, \infty}(T, P)}{R T}\right)
\end{array}\right\} .
$$

$\phi_{\dot{b}}^{\mathrm{L}, \infty}(T, P)$ denotes the fugacity coefficient of component $i$ at infinite dilution in solvent $j, G_{i}^{\mathrm{R}, \mathrm{L}, \infty}(T, P)$ denotes the partial molar isobaric residual Gibbs energy of component $i$ at infinite dilution, and $\mu_{i}^{\mathrm{R}, \mathrm{L}, \infty}(T, P)$ is its isobaric residual chemical potential at infinite dilution. Equation 310 provides an exact link between the thermodynamic description of any solution based on Henry's law and a description based on fugacity coefficients and thus on an equation of state: for solute 2 we have.

$$
h_{2,1}(T, P)=P \phi_{2}^{\mathrm{L}, \infty}(T, P) .
$$

In addition, using Eqs. 129, 132, 293, 297 and 310 as needed, the following rigorous relations between quantities used in a description based on activity coefficients and one based on fugacity coefficients (and hence on an EOS), are obtained (all quantities refer to the same temperature and pressure):

$$
\begin{aligned}
& \gamma_{i}^{\mathrm{LR}}=\phi_{i}^{\mathrm{L}} / \phi_{i}^{\mathrm{L}, *}, \\
& \gamma_{i}^{\mathrm{HL}}=\phi_{i}^{\mathrm{L}} / \phi_{i}^{\mathrm{L}, \infty},
\end{aligned}
$$

and in the limit $x_{i} \rightarrow 0$, for the LR activity coefficient at infinite dilution we have

$$
\lim _{x_{i} \rightarrow 0} \gamma_{i}^{\mathrm{LR}} \equiv \gamma_{i}^{\mathrm{LR}, \infty}=\gamma_{i}^{\mathrm{LR}} / \gamma_{i}^{\mathrm{HL}}=h_{i, j} / f_{i}^{\mathrm{L}, *}=\phi_{i}^{\mathrm{L}, \infty} / \phi_{i}^{\mathrm{L}, *} .
$$

For the sake of a more compact notation, the specifications $\left(T, P, x_{i}\right)$ etc., have been omitted. Equations 310-314 allow straightforward transition of system description from one using fugacity coefficients to one using activity coefficients, and vice versa.

The last topic I shall discuss briefly in this review concerns the determination of partial molar enthalpy changes on solution $\Delta H_{2}^{\infty}$ and partial molar heat capacity changes on solution $\Delta C_{P, 2}^{\infty}$ of sparingly soluble gases in liquids. The latter quantity is defined by

$$
\Delta C_{P, 2}^{\infty}(T, P) \equiv C_{P, 2}^{\mathrm{R}, \mathrm{L}, \infty}(T, P)=C_{P, 2}^{\mathrm{L}, \infty}-C_{P, 2}^{\mathrm{pg}, *}=\left(\frac{\partial \Delta H_{2}^{\infty}(T, P)}{\partial T}\right)_{P},
$$


where $C_{P, 2}^{\mathrm{L}, \infty}$ is the partial molar heat capacity at constant pressure of the solute at infinite dilution in the liquid solvent, and $C_{P, 2}^{\mathrm{pg}, *}$ is the molar heat capacity at constant pressure of the pure solute in the perfect-gas state. The notation $\Delta C_{P, 2}^{\infty}$ is generally preferred over $C_{P, 2}^{\mathrm{R}, \mathrm{L}, \infty}$, the partial molar residual isobaric heat capacity at infinite dilution $[66,169]$.

In fact, until quite recently high-precision measurements of Henry fugacities over temperature ranges large enough to permit van't Hoff analysis of the solubility data, constituted the only reliable source of information on these quantities [211, 214, 240, 241]. Once experimental Henry fugacities $h_{2,1}\left(T, P_{\sigma, 1}(T)\right)$ for a binary solution have been determined over a reasonably large temperature range (but not too close to the critical temperature of solvent 1), the most popular correlating equations are either the Clarke-Glew (CG) equation [242-244]

$$
\ln \left[h_{2,1}\left(T, P_{\sigma, 1}(T)\right) / \mathrm{Pa}\right]=A_{0}+A_{1}(T / \mathrm{K})^{-1}+A_{2} \ln (T / \mathrm{K})+\sum_{i=3}^{n} A_{i}(T / \mathrm{K})^{i-2},
$$

or the Benson-Krause (BK) equation [245-247], conventionally expressed as

$$
\ln \left[h_{2,1}\left(T, P_{\sigma, 1}(T)\right) / \mathrm{Pa}\right]=\sum_{i=0}^{n} a_{i}(T / \mathrm{K})^{-i} .
$$

Based on the ability to fit high-precision Henry fugacity data over fairly large temperature ranges, and of simplicity, the BK power series in 1/T appears to be superior. In passing I note that the 3-term version of Eq. 316 is the well-known Valentiner equation [248].

At this juncture I would like to emphasize once more that the frequently found sweeping statement "the solubility of a gas in a liquid decreases with increasing temperature" is misleading/incorrect when the entire liquid range of the solvent between its triple point temperature and its critical temperature is considered. For many binary solutions of gases in liquids, the following behavior is well documented [1, 66, 157, 169, 216, 249-251]: at low temperatures, the Henry fugacity $h_{2,1}\left(T, P_{\sigma, 1}\right)$ typically increases with increasing temperature, passes through a maximum, and then decreases towards its limiting value at the solvent's critical point (with critical temperature $T_{\mathrm{c}, 1}$ and critical pressure $P_{\mathrm{c}, 1}$ ), which, according to Eq. 311, is given by [66, 157, 169, 216-219]

$$
\lim _{\substack{T \rightarrow T_{\mathrm{c}, 1} \\ P_{\sigma, 1} \rightarrow P_{\mathrm{c}, 1}}} h_{2,1}\left(T, P_{\sigma, 1}\right)=P_{\mathrm{c}, 1} \phi_{2}^{\mathrm{L}, \infty}\left(T_{\mathrm{c}, 1}, P_{\mathrm{c}, 1}\right)=P_{\mathrm{c}, 1} \phi_{2}^{\mathrm{V}, \infty}\left(T_{\mathrm{c}, 1}, P_{\mathrm{c}, 1}\right) .
$$

Figure 3 provides two examples of such a solubility behavior: Henry fugacities, i.e. $\ln \left[h_{2,1}\left(T, P_{\sigma, 1}\right) / \mathrm{GPa}\right]$, of methane dissolved in liquid water, and of krypton dissolved in liquid water, are plotted against temperature [249, 252]. Note that the system $\left\{x_{1} \mathrm{H}_{2} \mathrm{O}+x_{2} \mathrm{CH}_{4}\right\}$ is important in the discussion of hydrophobic effects [253-262].

While the Henry fugacity remains finite at $T_{\mathrm{c}, 1}$, for volatile solutes the limiting slope approaches $-\infty$ when the critical point of the solvent is approached along the coexistence curve [263]:

$$
\lim _{\substack{T \rightarrow T_{\mathrm{c}, 1} \\ P \rightarrow P_{\mathrm{c}, 1}}} \frac{\mathrm{d} \ln \left[h_{2,1}\left(T, P_{\sigma, 1}\right) / \mathrm{Pa}\right]}{\mathrm{d} T}=-\infty
$$

Since the experimental Henry fugacities at different temperatures refer to different vapor pressures $P_{\sigma, 1}(T)$, we obtain with Eqs. 304 and 305 [66, 157, 169, 216, 219, 237-239] 


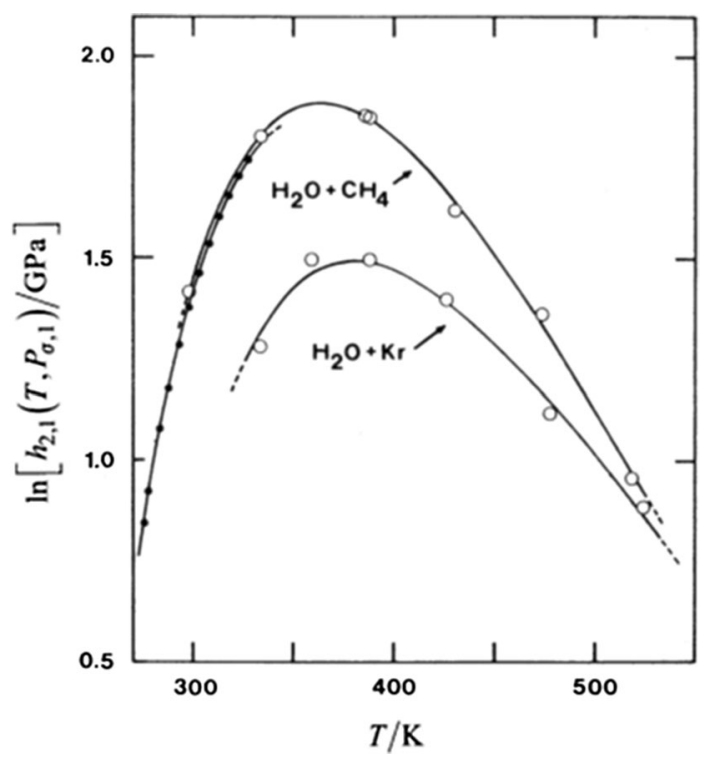

Fig. 3 Plot of $\ln \left[h_{2,1}\left(T, P_{\sigma, 1}\right) / \mathrm{GPa}\right]$ against temperature $T$ for krypton and methane dissolved in liquid water: $h_{2,1}\left(T, P_{\sigma, 1}\right)$ denotes the Henry fugacity (Henry's law constant) at temperature $T$ and corresponding pressure $P_{\sigma, 1}(T)$, the vapor pressure of water [108]. Open circles represent experimental results of Crovetto et al. [249]: the average percentage deviation of the Henry fugacities from the values calculated via BKtype fitting equations is about $\pm 2 \%$. Filled circles represent experimental results of Rettich et al. [252]: the average percentage deviation of the Henry fugacities from the values calculated via a correlating BK function, Eq. 317 , is about $\pm 0.05 \%$. The temperature where the Henry fugacity exhibits a maximum is about $382 \mathrm{~K}$ for $\left\{\mathrm{H}_{2} \mathrm{O}+\mathrm{Kr}\right\}$, and about $363 \mathrm{~K}$ for $\left\{\mathrm{H}_{2} \mathrm{O}+\mathrm{CH}_{4}\right\}$. The limiting values of the respective Henry fugacities $h_{2,1}\left(T, P_{\sigma, 1}\right)$ as $T \rightarrow T_{\mathrm{c}, 1}$ and $P_{\sigma, 1} \rightarrow P_{\mathrm{c}, 1}$ are finite and are given by Eq. 318, the limiting slope of the curves is $-\infty$, see Eq. 319. The figure was reproduced from E. Wilhelm, J. Solution Chem. 43, 525-576 (2014) [157]

$$
\begin{aligned}
\frac{\Delta H_{2}^{\infty}\left(T, P_{\sigma, 1}\right)}{R T} & =-T \frac{\mathrm{d} \ln \left[h_{2,1}\left(T, P_{\sigma, 1}\right) / \mathrm{Pa}\right]}{\mathrm{d} T} \\
& +\frac{V_{2}^{\mathrm{L}, \infty}}{R} \frac{\mathrm{d} P_{\sigma, 1}}{\mathrm{~d} T},
\end{aligned}
$$

and by analogous arguments

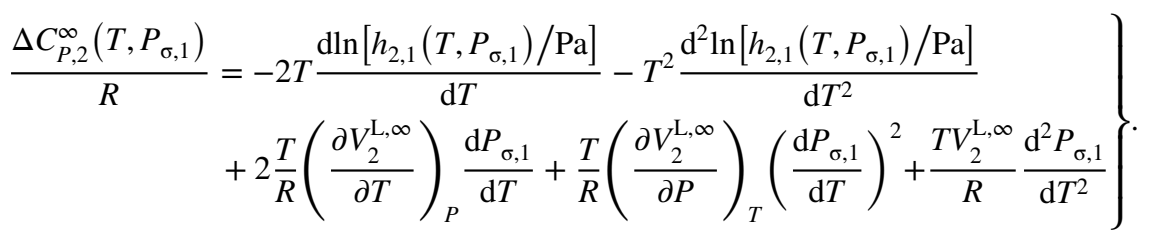

The second term on the right-hand side of Eq. 320 and the third, fourth and fifth term on the right-hand side of Eq. 321, respectively, containing the partial molar volume of the solute at infinite dilution $V_{2}^{\mathrm{L}, \infty}$ and its derivatives with respect to $T$ and $P$, together with $\mathrm{d} P_{\sigma, 1} / \mathrm{d} T,\left(\mathrm{~d} P_{\sigma, 1} / \mathrm{d} T\right)^{2}$ and $\mathrm{d}^{2} P_{\sigma, 1} / \mathrm{d} T^{2}$, are referred to in the literature as Wilhelm terms 
[247, 263, 264]. For aqueous solutions, say, of the rare gases below $373 \mathrm{~K}$, their contributions are small [247], usually smaller than the experimental error associated with current high-precision measurements, though they become more important in the critical region. World-wide, there were essentially only two groups that could provide the necessary highprecision experimental data on $h_{2,1}\left(T, P_{\sigma, 1}\right)$ with an imprecision of, say, $\pm 0.05 \%$, that allow reliable van 't Hoff-type analysis: the group of B. B. Benson and D. Krause, Jr. (BK), Department of Physics, Amherst College, Amherst, MA, USA, and the group of R. Battino, E. Wilhelm and T. R. Rettich (BWR), Department of Chemistry, Wright State University, Dayton, OH, USA.

Direct calorimetric determinations of the high-dilution molar enthalpy change on solution $\Delta_{\text {sol }} H$ of a gas in a liquid have been carried out by only a very limited number of researchers [265-277], simply because of the experimental difficulties associated with accurate measurements of very small heat effects in very dilute solutions (typically, aqueous mole fraction solubilities at ambient temperature and $0.1 \mathrm{MPa}$ gas pressure are about $10^{-5}$ to $10^{-4}$ ). Recently, various calorimeter designs used in this field were discussed in detail by Wilhelm and Battino [241]. The most prominent methodological step forward in measuring enthalpies of solution of gases in liquids with a precision high enough to allow reliable determination of the corresponding molar heat capacity changes on solution

$$
\Delta_{\mathrm{sol}} C_{P}=\left(\frac{\partial \Delta_{\mathrm{sol}} H}{\partial T}\right)_{P},
$$

is closely connected with the development of microcalorimeters in the Thermochemistry Laboratory of the University of Lund, Sweden (I. Wadsö's group), and in the Chemistry Department of the University of Colorado in Boulder, CO, USA (S. J. Gill's group), respectively. A fortiori, because of the formidable experimental difficulties encountered in the case of direct measurements of the molar heat capacity changes on solution of nonreacting gases dissolved in water at very low concentration, there exist only five sets of such data, all originating from the laboratory of R. H. Wood at the University of Delaware in Newark, DE, USA. Wood and collaborators determined the apparent molar isobaric heat capacities at very small solute mole fractions of the following four gaseous solutes in water: argon [278, 279], xenon [279], methane [280] and ethene [279]; in addition, they measured the apparent molar isobaric heat capacities at small mole fractions of aqueous solutions of $\mathrm{CO}_{2}, \mathrm{H}_{2} \mathrm{~S}$ and $\mathrm{NH}_{3}$ [280]. The sophisticatedly constructed flow calorimeter developed by Wood and his collaborators was used over very large temperature ranges from about $305 \mathrm{~K}$ to well into the supercritical region of water up to $T \approx 720 \mathrm{~K}$. Measurements of the apparent molar heat capacities of acid gases in liquid water near ambient conditions using a commercial Picker calorimeter [281] were reported by Barbero et al.: aqueous solutions of hydrogen sulfide at $10^{\circ} \mathrm{C}, 25^{\circ} \mathrm{C}$ and $40{ }^{\circ} \mathrm{C}$ [282]; aqueous solutions of carbon dioxide and sulfur dioxide at $25{ }^{\circ} \mathrm{C}[283]$; note that $T / \mathrm{K}=t /{ }^{\circ} \mathrm{C}+273.15$.

Although the measurements of Wood et al. were all performed at somewhat elevated pressures between, roughly, $17 \mathrm{MPa}$ and $32 \mathrm{MPa}$, the mole fractions of the dissolved gases are still small enough to make the apparent molar heat capacities to a good approximation, that is, within experimental error, equal to the partial molar isobaric heat capacities at infinite dilution (this statement is valid at temperatures below ca. $500 \mathrm{~K}$ ). At constant $T$ and $P$ we have 


$$
C_{P, 2, \mathrm{app}}^{\mathrm{L}} \equiv \frac{n C_{P}^{\mathrm{L}}-n_{1} C_{P, 1}^{\mathrm{L}, *}}{n_{2}}
$$

hence

$$
C_{P, 2}^{\mathrm{L}}=C_{P, 2, \mathrm{app}}^{\mathrm{L}}+n_{2}\left(\frac{\partial C_{P, 2, \mathrm{app}}^{\mathrm{L}}}{\partial n_{2}}\right)_{T, P, n_{1}},
$$

Since

$$
\lim _{n_{2} \rightarrow 0} C_{P, 2, \mathrm{app}}^{\mathrm{L}}=C_{P, 2}^{\mathrm{L}, \infty}
$$

we obtain

$$
\Delta C_{P, 2}^{\infty}(T, P) \cong C_{P, 2, \mathrm{app}}^{\mathrm{L}}(T, P)-C_{P, 2}^{\mathrm{pg}, *}(T)=\Delta C_{P, 2}^{\infty}(\text { Wood }) .
$$

Except for the few sets of direct heat capacity data on aqueous solutions of nonreacting gases as reported by Wood et al., all other calorimetry-based determinations of molar heat capacity changes on solution were obtained from the temperature dependence of the enthalpies of solution via Eq. 322.

Evidently, comparing van't Hoff derived enthalpy changes (one differentiation level, see Eq. 320) and heat capacity changes (two differentiation levels, see Eq. 321) with directly obtained high-precision calorimetric results constitutes a particularly severe test of solubility data. Recently, Wilhelm [169], Wilhelm and Battino [240, 241], and Battino [284] presented essentially comprehensive compilations of van 't Hoff-based partial molar enthalpy changes on solution $\Delta H_{2}^{\infty}$ and partial molar heat capacity changes on solution $\Delta C_{P, 2}^{\infty}$ for gases dissolved in liquid water at $T=298.15 \mathrm{~K}$ and $P_{\sigma, 1}\left(\mathrm{H}_{2} \mathrm{O} ; 298.15 \mathrm{~K}\right)=3.1691 \mathrm{kPa}$, and compared them with calorimetrically determined results for $\Delta_{\mathrm{sol}} H$ and $\Delta_{\mathrm{sol}} C_{P}$ at high dilution and ambient pressure. Note that high-precision solubility data for nitrogen and carbon monoxide dissolved in water [285, 286] are available, but somewhat surprisingly no calorimetric results have been reported so far. For the sake of brevity, in Table 5 I have limited such a comparison to a few representative systems at $298.15 \mathrm{~K}$, though essentially the same overall picture emerges for other systems at other temperatures too: agreement between these two approaches is highly satisfactory, i.e., it is usually within the combined experimental error: what a credit to experimental ingenuity and to the skills of solution thermodynamicists!

\section{Concluding Remarks, Future Directions and Acknowledgments}

By common consent, the liquid state of matter houses by far the largest group of unsolved/ crudely solved problems in modern physical chemistry, especially when bio-physical chemistry is included: indeed, molecular thermodynamics of aqueous solutions of biorelevance is an exciting, dynamically evolving field. With increasingly sophisticated computer simulations, new insights at microscopic, mesoscopic, and macroscopic levels are obtained, providing stimulating connections with a growing number of neighboring fields, in particular with bio-medical research. Another major driving force is the unabated progress in instrumentation: increasing the applicability range, that is, larger temperature 
Table 5 Comparison of partial molar enthalpy changes on solution $\Delta H_{2}^{\infty}\left(T, P_{\sigma, 1}\right)$ and partial molar heat capacity changes on solution $\Delta C_{P, 2}^{\infty}\left(T, P_{\sigma, 1}\right)$ determined via van't Hoff-type data treatment of solubilities of gases in liquid water (that is, Henry fugacities) with molar enthalpies of solution $\Delta_{\text {sol }} H$ and molar heat capacity changes on solution $\Delta_{\mathrm{sol}} C_{P}$ determined via calorimetry at high dilution and ambient pressure, and with partial molar heat capacity changes on solution $\Delta C_{P, 2}^{\infty}$ (Wood), obtained calorimetrically by Wood et al. [278-280] via Eq. 326: selected literature values for five representative gases dissolved in water at $298.15 \mathrm{~K}$

\begin{tabular}{|c|c|c|c|c|c|}
\hline \multirow[t]{2}{*}{ Gas } & \multicolumn{2}{|c|}{$\begin{array}{l}\text { From the solubility of gases in water: } \\
\text { van't Hoff analysis }\end{array}$} & \multicolumn{3}{|c|}{ From calorimetry } \\
\hline & $\frac{\Delta H_{2}^{\infty}\left(T, P_{\sigma, 1}\right)}{\mathrm{kJ} \cdot \mathrm{mol}^{-1}}$ & $\frac{\Delta C_{P, 2}^{\infty}\left(T, P_{\sigma, 1}\right)}{\mathrm{J} \cdot \mathrm{K}^{-1} \cdot \mathrm{mol}^{-1}}$ & $\frac{\Delta_{\mathrm{sol}} H}{\mathrm{~kJ} \cdot \mathrm{mol}^{-1}}$ & $\frac{\Delta_{\mathrm{sol}} C_{P}}{\mathrm{~J} \cdot \mathrm{K}^{-1} \cdot \mathrm{mol}^{-1}}$ & $\frac{\Delta C_{P, 2}^{\infty}(\text { Wood })}{\mathrm{J} \cdot \mathrm{K}^{-1} \cdot \mathrm{mol}^{-1}}$ \\
\hline $\mathrm{Ar}$ & -11.96 [287] & 192 [287] & -12.01 [269] & 200 [269] & $185[278]$ \\
\hline $\mathrm{O}_{2}$ & -11.97 [288] & $200[288]$ & -12.00 [269] & 205 [269] & \\
\hline $\mathrm{CH}_{4}$ & -13.19 [252] & 237 [252] & -13.12 [273] & 209 [273] & 212 [280] \\
\hline $\mathrm{C}_{2} \mathrm{H}_{6}$ & $-19.50[252]$ & $270[252]$ & $-19.43[274]$ & $273[274]$ & \\
\hline $\mathrm{C}_{2} \mathrm{H}_{4}$ & -16.40 [289] & 239 [289] & $-16.46[270]$ & 237 [272] & 221 [279] \\
\hline
\end{tabular}

At this temperature, the vapor pressure of water is $P_{\sigma, 1}=3.1691 \mathrm{kPa}[108]$

and pressure ranges, ever smaller concentrations, improving precision and accuracy, and increasing the speed of measurements together with facilitating application and data transfer now allows tackling problems which, say, twenty years ago would have been considered to be quite impossible tasks. In this review, I have focused on the rigorous and concise presentation of the thermodynamic formalism relevant for the study of liquid solutions of nonelectrolytes, in particular of dilute liquid solutions, and I have tried to cover, in a coherent fashion, the most important aspects of its practical implementation, indicating areas where more and/or more detailed experimental work would be desirable. Special emphasis was placed on binary aqueous solutions of supercritical solutes (gases), a field I have worked in with Rubin Battino for more than 50 years. Two closely related areas have been dealt with prominently:

- discussion of solution behavior in terms of the Henry fugacity $h_{2,1}\left(T, P_{\sigma, 1}\right)$ (also known as Henry's law constant) and related quantities, such as fugacity coefficients and activity coefficients, and exposing some frequently encountered misconceptions;

- discussion of the equivalency of results for caloric quantities (e.g., $\Delta H_{2}^{\infty}$ and $\Delta C_{P, 2}^{\infty}$ ) derived from solubility measurements via van't Hoff analysis with those measured directly with calorimeters.

These two topics present the state of the art for the (potential) experimentalist. Results obtained for aqueous systems by the BK and BWR groups (VLE), by Wadsö et al. and by Gill et.al. (calorimetry), have greatly contributed to our understanding and appreciation of hydrophobic effects. They are thought to play an important role in biology, though in many complex biochemical processes hydrophilic effects may be more important [290, 291]. A rich research field lies ahead, as evidenced by the increasing number of investigations with a strong biophysical and/or biomedical flavor. The research of Wood et al., based on the instruments he developed, made possible pioneering measurements of the density and the heat capacity of dilute aqueous solutions of nonelectrolytes as well as electrolytes from ambient temperatures to the critical temperature of water, $T_{\mathrm{c}}=647.1 \mathrm{~K}$, and beyond, well into the single fluid region, yielding novel, spectacular results [292]. 
What I hope to have also communicated is my conviction that advances in science inevitably lead to a broadening and merging of neighboring areas of research, and that crossdisciplinary fundamental research is always a potent stimulus in science as well as for technological innovation.

Studies on solubility in general, and on solubility in liquid water in particular, have come a long way. The field has grown too big to be covered in one modest review article, but for the foundations and the few subsections covered I hope to have succeeded in providing a feeling for their scope, current position in physical chemistry, and future potential. Related to these aspects I find the statement by Gilbert Newton Lewis (1875-1946) on the practical philosophy of science most appropriate and encouraging. In fact, it is one of my favorite quotes, and although I've cited it before, the insight contained makes it appropriate to repeat it here:

The scientist is a practical man and his are practical aims. He does not seek the ultimate but the proximate. He does not speak of the last analysis but rather of the next approximation.... On the whole, he is satisfied with his work, for while science may never be wholly right it certainly is never wholly wrong; and it seems to be improving from decade to decade.

My view of chemical thermodynamics has evolved over the years, and I have benefitted from collaborations as well as discussions with many friends and colleagues. Quite early in my career I was asked to participate at various IUPAC activities, and I did indeed devote an appreciable part of my "scientific life" to further the Union's goals, both nationally and internationally (see Appendix). Thus, I was particularly pleased to participate, as an Invited Lecturer, at the 47th IUPAC World Chemistry Congress: Frontiers in Chemistry, celebrating 100 Years with IUPAC, in Paris, France, 7-12 July 2019. This Invited Lecture was part of a Special Symposium dedicated to Professor Eduard Hála (1919-1989), who was one of the great pioneers of modern vapor-liquid experimental science and theory. Incidentally, Professor Hála and I were both founding members of the editorial board of Fluid Phase Equilibria, one of the internationally leading journals in this field. This Special Symposium was admirably organized by M. Bendova, ICPF/CAS, Prague, Czech Republic, and J. Jacquemin, Université de Tours, Tours, France.

Chemical thermodynamics is a vast field of science with many applications to realworld problems (hence many advances originated from work of chemical engineers). For me it is a great pleasure to acknowledge here (a) the initiation to this topic, many decades ago, by Professor Friedrich Kohler ( $\dagger$ ), my $\mathrm{PhD}$ advisor (on adiabatic calorimetry and applied statistical mechanics) at the University of Wien, Austria, via his courses and seminars [293]; (b) the more than 50 years of fruitful scientific collaboration with Rubin Battino, now Emeritus Professor at Wright State University (WSU), Dayton, Ohio, USA, who introduced me to the fascinating field of the solubility of gases in liquids; (c) the work with Jean-Pierre E. Grolier, now Emeritus Professor at Université Blaise Pascal, ClermontFerrand, France, with whom I enjoyed about 45 years of exciting scientific collaboration with more than 25 years of continuously funded research in solution thermodynamics (that is, research on $H^{\mathrm{E}}, C_{P}^{\mathrm{E}}$ and $V^{\mathrm{E}}$ ), and many memorable first-class dinners; (d) the cooperation with Henry V. Kehiaian $(\dagger)$ [294, 295], former Directeur de Recherches at ITODYS (CNRS), Paris, France, and an old friend since 1967 (since his first visit from Warsaw, Poland, to the"west", that is, to the Institute of Physical Chemistry, University of Wien, Vienna, Austria), on group contribution theory and many enjoyable dinners (often together with Jean-Pierre Grolier); (e) the research (since 1981) with Augustinus Asenbaum, now Retired Professor of Experimental Physics at the University of Salzburg, Salzburg, Austria, 
who opened the doors to the fascinating extra-thermodynamic field of Brillouin scattering at ambient and elevated pressures; and (f) the satisfying and frequently stimulating work with about 80 colleagues, post-doctoral fellows and students from 17 countries. Without them, many projects would have been difficult to carry out. Fortunately, over the years the University of Wien (Vienna), Austria, was generous enough to grant repeatedly extended Leaves of Absence for scientific research abroad, and in the present context, the most important were two extended stays in the United States of America at WSU, Dayton, Ohio (first as a Senior Fulbright Research Associate, then as Visiting Associate Professor: about four years altogether), which I greatly enjoyed. In fact, most of the experimental work on gas solubility was supported by the Public Health Service of the United States of America via grants from the National Institute of General Medical Sciences, and a smaller part was supported by the Petroleum Research Fund, administered by the American Chemical Society. Research on fluid phase chemical thermodynamics funded within the frame of the Austrian-French Program on Scientific and Technical Cooperation also led to many extended stays in France, altogether for more than five years: first at the CRMT/CNRS in Marseille (Tian-Calvet Institute), and then at the Université Blaise Pascal in Clermont-Ferrand and at ITODYS in Paris, all of which I also greatly enjoyed. When visiting Jean-Pierre Grolier in Clermont-Ferrand, I frequently had also the pleasure to meet Robert H. Wood on leave from the University of Delaware, Newark, DE, USA, to whom a Special Issue of The Journal of Solution Chemistry (Volume 44, Number 5), a Festschrift to celebrate his eightieth birthday, was dedicated in May 2015. These joint stays provided a welcome opportunity to discuss molecular thermodynamics problems in general, and hot topics in solution thermodynamics in particular. In appreciation of our scientific research activities at the Université Blaise Pascal, Bob Wood and I were recipients of honorary doctorates. Besides science, for me those years in Marseille, Clermont-Ferrand and Paris provided unforgettable experiences associated with France: indeed, these were the years of St. Nectair and Bleu d'Auvergne, of Saint-Emilion and Pomerol!

\section{Appendix: Some of My IUPAC-Related Activities}

\section{IUPAC Solubility Data Series}

IUPAC Commission V.8 (Solubility Data)

IUPAC Commission I.2 (Thermodynamics)

IUPAC Committee on Legendre Transforms

IUPAC-CODATA Commission IUCOSPED IUPAC Fellow founding Member of the Editorial Board and Topic Editor, 1976-1981.

Associate Member, and/or National Representative, and/or Member of Subcommittee on Gas Solubilities, 1977-2001.

Associate Member, 1985-1993.

Member (with R. A. Alberty ( $\dagger$ ), Chairman, J. M. G. Barthel (†), E. R. Cohen, M. B. Ewing ( $\dagger$ ), and R. N. Goldberg), 1994 2001. Recommendations published as: IUPAC Technical Report: Use of Legendre Transforms in Chemical Thermodynamics, Pure Appl. Chem. 73, 1349-1380 (2001).

Member, 1999-2003.

Since 2010 
Co-organizer of:>

$\begin{array}{lcc}\text { 3rd ICCT } & \text { Baden near Vienna, Austria, } 1973 . \\ \text { 12th ICCT } & \text { Snowbird, Utah, USA, 1992. } \\ \text { 17th ICCT } & \text { Rostock, Germany, 2002. } & \\ \text { 19th ICCT (THERMO International, +61st CALCON + 16th STP) } & \text { Boulder, CO, } \\ & \text { USA, 2006. } \\ \text { 21st ICCT } & \text { Tsukuba Science City, Ibaraki, Japan, } 2010 .\end{array}$

Plenary Lectures/Invited Lectures:

6th ICCT

8th ICCT + 39th CALCON

1st International Symposium on Solubility Phenomena London, Ontario, Canada, 1984. Journées d'étude sur les capacités calorifiques des systèmes condensés (Societé Française de Chimie \& IUPAC)

11th ICCT

13th ICCT

14th ICCT

26th International Conference on Solution Chemistry Fukuoka, Japan, 1999.

IUPAC-CODATA Meeting IUCOSPED

IUPAC-CODATA Meeting IUCOSPED

18th ICCT

25th ICCT + 73rd CALCON

Cadarache, France, 1986.

Como, Italy, 1990.

Laudatory Speech in honor of Prof. H. C.

Van Ness at the occasion of his Rossini Lecture/Award, Clermont-Ferrand, France, 1994. Osaka, Japan, 1996.

Berlin, Germany, 1999.

Paris, France, 2001.

Beijing, China, 2004.

Granlibakken/Lake Tahoe, CA, USA, 2018.

47th IUPAC World Chemistry Congress/100 years with IUPAC Paris, France, 2019.

Co-editor, with Prof. Trevor M. Letcher, of Four Books:

Heat Capacities

Volume Properties

Enthalpy and Internal Energy

Gibbs Energy and Helmholtz Energy
Liquids, Solutions and Vapours. The Royal Society of Chemistry/IUPAC \& IACT, Cambridge (2010). Liquids, Solutions and Vapours. The Royal Society of Chemistry/IUPAC \& IACT, Cambridge (2015). Liquids, Solutions and Vapours. The Royal Society of Chemistry/IACT, London (2018).

Liquids, Solutions and Vapours. The Royal Society of Chemistry/IACT, London (2021).

\section{Glossary of Symbols}

In most cases I have adhered to the nomenclature/symbols suggested by the International Union of Pure and Applied Chemistry, IUPAC (see Quantities, Units and Symbols in Physical Chemistry [296], commonly known as the Green Book). Deviations from a few of these suggestions are due to my desire to present a concise, unequivocal and logically consistent notation in compliance with usage preferred by the scientific community interested in this review's topics, that is, by physical chemists, physicists and chemical engineers. 
Such an approach is in accord with the spirit of the Green Book expressed so admirably by Martin Quack in his Historical Introduction on p. XII of its 3rd edition, 2007: It is not the aim to present a list of recommendations in form of commandments. Rather we have always followed the principle that this manual should help the user in what may be called "good practice of scientific language". However, IUPAC suggestions differing from those used here I have included in 5.1 List of Symbols.

A few quantities I would like to single out to comment on are: the pressure $P$, the mechanical coefficients, i.e., the isobaric expansivity $\alpha_{P}$, the isothermal compressibility $\beta_{T}$, and the isochoric thermal pressure coefficient $\gamma_{V}$, and the Henry fugacity $h_{i, j}(T, P)$, also known as Henry's law constant.

The symbol $P$ for pressure is now accepted by IUPAC as an alternative to $p$, as indicated in Tables 2.2 and 2.10 of the Green Book. The reason why I (and many others) prefer $P$ is the following: temperature and pressure are both intensive quantities, and together with the composition expressed by the set of mole fractions $\left\{x_{i}\right\}$, or by the set of amounts of substance $\left\{n_{i}\right\}$, they form a group of basic thermodynamic variables advantageously used in solution chemistry. They are not perceived primarily as properties of the fluids but as conditions imposed on/exhibited by them with the valuable bonus of being (in principle) easily measured and controlled. In other words, temperature and pressure are quantities of "equal rank", which fact should be reflected in the symbols we use, that is, capital $T$ and capital $P$. For heterogeneous PVTx systems consisting of several phases in equilibrium with each other, temperature and pressure are identical in the coexisting phases. Griffiths and Wheeler [297] call such variables fields (in contradistinction to variables that are in general not equal in coexisting phases, such as molar volume, molar enthalpy, and molar entropy, which they call densities). For the isothermal compressibility, Rowlinson and Swinton [29], amongst many others, use the symbol $\beta_{T} \equiv-V^{-1}(\partial V / \partial P)_{T,\left\{x_{i}\right\}}$. Together with the isobaric expansivity $\alpha_{P} \equiv V^{-1}(\partial V / \partial T)_{P,\left\{x_{i}\right\}}$ and the isochoric thermal pressure coefficient $\gamma_{V} \equiv(\partial P / \partial T)_{V,\left\{x_{i}\right\}}$, for a constant-composition fluid, and thus also for pure fluids, these mechanical coefficients form a mnemonic triple:

$$
\alpha_{P} / \beta_{T}=\gamma_{V}
$$

Writing them this way, i.e., by indicating via subscript what quantity is to be held constant, is advantageous in general, and particularly so when discussing the related isentropic and orthobaric quantities [298]. The Henry fugacity $h_{i, j}(T, P)$ depends on $T$ and $P$, and also on the chemical identities of solute $i$ and solvent $j$ (the other component), hence the double subscript $i, j$ has been added to the symbol $h$. The Henry fugacity (hence the lower-case letter $h$ ) is a material property $[66,169]$, which fact is clearly indicated by Eq. 310 .

Some of the symbols listed below may be modified further, with obvious meaning, by adding appropriate subscripts, such as $\sigma$ (saturation or orthobaric condition), and/or superscripts, such as $*$ (pure substance), $\infty$ (infinite dilution), and L (liquid) or V (vapor). The capital superscript letters are used because (I) they are easy to read, (II) they are frequently used in the chemical engineering literature, including important monographs (for instance, Prausnitz et al. [1], Poling et al. [49]) and volumes published under the auspices of the International Union of Pure and Applied Chemistry (IUPAC) [155, 158, 160, 169, 172], and (III) vapor-liquid equilibrium is usually abbreviated by VLE, and not by vle.

Finally, a very recent important development that has already been pointed out in footnote 2: please note that the values for some fundamental physical constants listed in the glossary below, such as the Avogadro constant $N_{\mathrm{A}}$, and the Boltzmann constant $k_{\mathrm{B}}$, are now exactly defined $[44,45]$. 


\section{List of Symbols}

\section{Variables and Constants}

$a$

$a_{i}$

$a_{i i}, a_{j j}$

$a_{i j}$

$A_{0}, A_{m}$

$A_{12}, A_{21}$

$A_{12}^{\prime}, A_{21}^{\prime}$

$A_{i}$

$b$
$b_{i i}, b_{j j}$
$b_{i j}$
$B$
$B, C, D$
$B^{\prime}, C^{\prime}, D^{\prime}$
$B_{n}$
$B_{11}, B_{22}$

$B_{11}, B_{22}$

$B_{12}$

$c$

$c_{\mathrm{PT}}$

$c_{\mathrm{PT} i i}, c_{\mathrm{PT} j j}$

$c_{\mathrm{PT} i j}$

$C_{c}^{p}$

$C_{P}$

$C_{P, i, *}^{*} C_{P, i}^{\mathrm{pg}, *}(T)$

$C_{P, i}$
Attractive interaction parameter, vdW-type equations of state, Eqs. 2, 162, 163, 165,179

$i=0,1,2, \ldots$ Dimensionless coefficients used to describe the temperature dependence of the Henry fugacity, i.e., of $\ln \left[h_{2,1}\left(T, P_{\sigma, 1}(T)\right) / \mathrm{Pa}\right]$, Eq. 317 (BK); or the temperature dependence of $G^{\mathrm{E}} / R T$ etc., Eqs. 268-271

Attractive interaction parameters, pure fluids (vdW-type equation of state): Eqs. 177 and 181

Cross-interaction parameter (vdW-type equation of state): Eqs. 177 and 181

Redlich-Kister parameters, Eq. 259, or Padé parameters, Eq. 261

van Laar parameters, Eq. 277

Margules parameters, Eq. 274

$i=0,1,2, \ldots$ Dimensionless coefficients used to describe the temperature dependence of the Henry fugacity, i.e., of $\ln \left[h_{2,1}\left(T, P_{\sigma, 1}(T)\right) / \mathrm{Pa}\right]$, Eq. 316 (CG)

Covolume, vdW-type equations of state, Eqs. 2, 162, 163, 165, 179

Repulsive interaction parameters, pure fluids (vdW-type equation of state): Eqs. 178 and 182

Cross repulsive interaction parameter (vdW-type equation of state): Eqs. 178 and 182

Second virial coefficient of a gaseous mixture, associated with an amount-density expansion of the compression factor Z, Eq. 184

Parameters of Eq. 273

Redlich-Kister parameters, Eqs. 272 and 280

Padé parameters, Eq. 261

Second virial coefficients of the pure components 1 and 2 in the gas phase/ vapor phase (corresponding to the amount-density expansion of the compression factor Z), respectively, Eqs. 153b, 153c and 248. They are used in the truncated volume-explicit two-term virial equation in pressure $Z_{i}^{\mathrm{V}, *} \equiv P V_{i}^{\mathrm{V}, *} / R T=1+B_{i i} P / R T, i=1$ or 2

Vapor-phase interaction virial coefficient (cross-coefficient), associated with a binary gas phase/vapor phase, Eqs. 153b, 153c and 248

Number of components in a multi-component solution

Parameter, Patel-Teja equation of state, Eq. 179

Parameter, pure fluids (Patel-Teja equation of state): Eqs. 180 and 183

Cross-parameter, Patel-Teja equation of state, Eqs. 180 and 183

$=\left(\begin{array}{l}p \\ c\end{array}\right)$, Combinations without repetition (order is not important), Eq. 1

Molar heat capacity at constant pressure (molar isobaric heat capacity)

Molar heat capacity at constant pressure of pure substance $i$

Molar heat capacity at constant pressure of pure substance $i$ in the perfect-gas (ideal-gas) state

Partial molar heat capacity at constant pressure of component $i$ of a mixture/ solution 
$C_{P}^{\mathrm{E}} \quad$ Molar excess heat capacity at constant pressure of a mixture/solution

Partial molar excess heat capacity at constant pressure of component $i$ of a mixture/solution

$C_{P, 2}^{\mathrm{L}, \infty} \quad$ Partial molar heat capacity at constant pressure of solute 2 at infinite dilution in a liquid (L) mixture/solution

$C_{P, 2, \text { app }}^{\mathrm{L}}$

$\Delta C_{P} \quad$ Molar heat capacity change of mixing

$\Delta C_{P .2}^{\infty} \quad$ Partial molar heat capacity change on solution of a gas (2) in a liquid (1) at infinite dilution

$\Delta_{\text {sol }} C_{P} \quad$ Molar heat capacity change on solution, calorimetrically determined

$C_{V}$

$f$

Molar heat capacity at constant volume (molar isochoric heat capacity)

$f_{i}^{\pi} \quad$ Fugacity of component $i$ in solution in phase $\pi$ (L or V)

$f_{i}^{\mathrm{L}, *}$

$f_{i}^{\mathrm{V}, *}$

$=\phi_{i}^{\mathrm{L}, *} P$, Fugacity of pure substance $i$ in the liquid phase

$=\phi_{i}^{\mathrm{V}, *} P$, Fugacity of pure substance $i$ in the vapor phase

$f^{(1)}, f^{(2)}$

First-order partial Legendre transform, second-order partial Legendre transform

$F$

Molar Helmholtz energy, see Table 1; the Green Book [296] suggests $F$ and $A$

$G$

Molar Gibbs energy, see Table 1

$G_{i}^{*} \quad$ Molar Gibbs energy of pure substance $i$

$G_{i}$

$=\mu_{i}$, Partial molar Gibbs energy of component $i$ of a mixture/solution, chemical potential of component $i$

$G^{\mathrm{E}} \quad$ Molar excess Gibbs energy of a mixture/solution, Eqs. 214 and 220

$G_{i}^{\mathrm{E}} \quad=\mu_{i}^{\mathrm{E}}$, Partial molar excess Gibbs energy of component $i$ of a mixture/solution, excess chemical potential of component $i$, Eqs. 219 and 237

$\Delta G \quad$ Molar Gibbs energy change of mixing

$h_{i, j}$ $=h_{i, j}(T, P)$, Henry fugacity (or Henry's law constant) of component $i$ dissolved in liquid solvent $j$. Usually, experimental data obtained via extrapolation $x_{i} \rightarrow 0$ are reported at pressures $P=P_{\sigma, j}$, the vapor pressure of the solvent, that is, $h_{i, j}\left(T, P_{\sigma, 1}\right)$ is reported, see Eq. 301. This nomenclature has been adopted in Volume VII of IUPAC's Experimental Thermodynamics Series, Measurement of the Thermodynamic Properties of Multiple Phases [169]. Prausnitz et al. [1] use the symbol $H_{i, j}$, and the Green Book of IUPAC [296] suggests $k_{\mathrm{H}, \mathrm{B}}$, where the definition applies to entities B which should be specified.

$H \quad$ Molar enthalpy, see Table 1

$H_{i}^{*} \quad$ Molar enthalpy of pure substance $i$

$H_{i}$

$H^{\mathrm{E}}$

Partial molar enthalpy of component $i$ of a mixture/solution

$H_{i}^{\mathrm{E}}$

$\Delta H \quad$ Molar enthalpy change of mixing

Molar excess enthalpy of a mixture/solution, Eqs. 217 and 223

$\Delta H_{2}^{\infty} \quad$ Partial molar enthalpy change on solution when dissolving a gas (2) in a liquid (1), at infinite dilution

$\Delta_{\text {sol }} H \quad$ Molar enthalpy change on solution, calorimetrically determined $\equiv U-T S-\sum_{i} \mu_{i} x_{i}=-P V$, Molar grand canonical potential, see Table 1; not mentioned in the Green Book [296]

$k_{\mathrm{B}} \quad=1.380649 \times 10^{-23} \mathrm{~J} \cdot \mathrm{K}^{-1}=R / N_{\mathrm{A}}$, Exactly, Boltzmann constant $[44,45]$

$k_{i j}$

Binary interaction parameter, Eq. 181 
$l_{i j} \quad$ Binary interaction parameter, Eq. 182

$L_{p}\left(z_{12}\right) \quad$ Legendre polynomial, Eq. $262, z_{12} \equiv x_{1}-x_{2}$

$m \quad$ Mass

$m_{\mathrm{m}} \quad=m / n$, Molar mass; $n$ denotes the total amount of substance

$m_{i j} \quad$ Binary interaction parameter, Eq. 183

$M$

$M^{\mathrm{t}} \quad \equiv n M$, Total thermodynamic property of a single-phase multicomponent solution (extensive property)

$M_{i}^{*} \quad$ Molar thermodynamic property of pure homogeneous fluid $i$

$M_{i}^{i} \quad$ Partial molar property of component $i$ of a mixture/solution

$M^{\mathrm{E}} \quad$ Molar excess property of a mixture/solution, Eq. 211

$M_{i}^{\mathrm{E}} \quad$ Partial molar excess property of component $i$ of a mixture/solution, Eq. 212

$\Delta M \quad$ Molar property change on mixing, Eq. 189

$\Delta M_{i} \quad$ Partial molar property change on mixing, Eq. 190

$\Delta_{1 \rightarrow 2} M \quad$ Property change for $1 \mathrm{~mol}$ of a thermodynamic property $M$ of a homogeneous fluid when going from $\left\{T_{1}, P_{1}\right\}$ to $\left\{T_{2}, P_{2}\right\}$, Eq. 156

$n$

$=\sum_{i}^{c} n_{i}$, Total amount (of substance) of a mixture/solution with $c$ components

$n_{i} \quad$ Amount (of substance) of component $i$ of a mixture/solution

$N \quad=n N_{\mathrm{A}}$, Number of molecules

$N_{\mathrm{A}}=6.02214076 \times 10^{23} \mathrm{~mol}^{-1}$, Exactly, Avogadro constant [44, 45]; the Green Book [296] suggests $L$ and $N_{\mathrm{A}}$

$N_{\text {Le,p }} \quad$ Total number of partial Legendre transforms, Eq. 49

$N_{\mathrm{Le}}^{\mathrm{t}}$

$P$

$P_{\mathrm{c}} \quad$ Critical pressure

$P_{\mathrm{r}} \quad \equiv P / P_{\mathrm{c}}$, Reduced pressure

$P_{\sigma} \quad$ Vapor pressure

$P_{\text {ref }} \quad$ Suitably selected reference pressure, frequently $10^{5} \mathrm{~Pa}$

$R$

$=N_{\mathrm{A}} k_{\mathrm{B}}=8.314462618 \mathrm{~J} \cdot \mathrm{K}^{-1} \cdot \mathrm{mol}^{-1}$, (molar) gas constant $[44,45]$

$S \quad$ Molar entropy, see Table 3

$S_{i}^{*} \quad$ Molar entropy of pure substance $i$

$S_{i}$

$S^{\mathrm{E}}$

$S_{i}^{\mathrm{E}}$

$\Delta S$

Partial molar entropy of component $i$ of a mixture/solution

Molar excess entropy of a mixture/solution, Eqs. 215 and 221

Partial molar excess entropy of component $i$ of a mixture/solution

Molar entropy change of mixing

$\Delta S_{2}^{\infty} \quad$ Partial molar entropy change on solution when dissolving a gas (2) in a liquid (1), at infinite dilution

$t \quad$ Celsius temperature, $t /{ }^{\circ} \mathrm{C}=T / K-273.15$

$T \quad$ Thermodynamic temperature

$T_{\mathrm{c}} \quad$ Critical temperature

$T_{\mathrm{r}} \quad \equiv T / T_{\mathrm{c}}$, Reduced temperature

$T_{\text {ref }} \quad$ Suitably selected reference temperature

$T_{\text {tr }} \quad$ Triple point temperature

$u(r) \quad$ Intermolecular pair-potential energy function, Eq. 82

$U \quad$ Molar internal energy, see Table 1

V Molar volume 


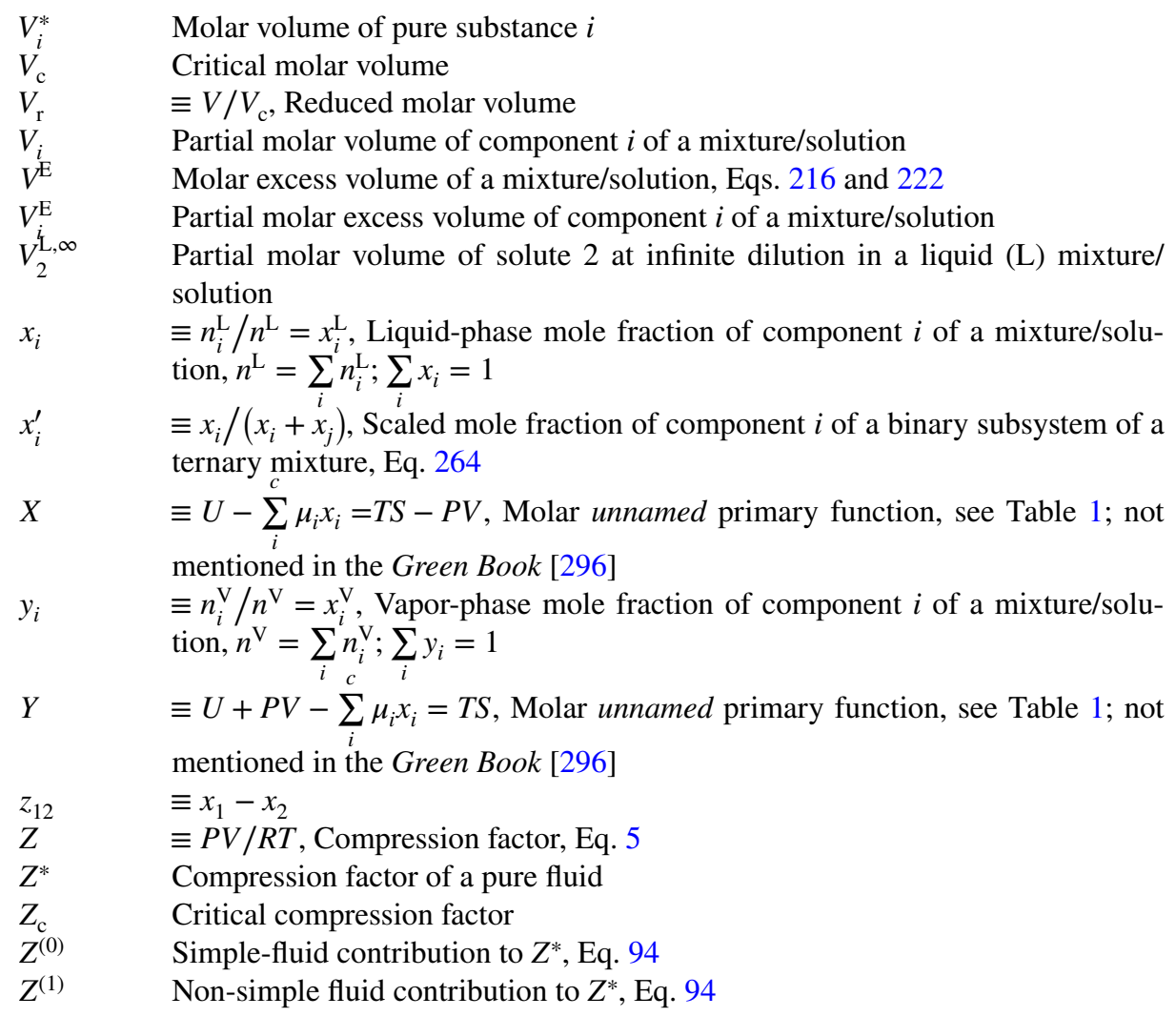

\section{Greek letters}

$\alpha(\tau, \delta) \quad=F\left(T, \rho_{n}\right) / R T=\alpha^{\mathrm{r}}(\tau, \delta)+\alpha^{\mathrm{pg}}(\tau, \delta), \quad$ Empirical fundamental equation, Eq. 187

$\alpha_{P} \quad \equiv V^{-1}(\partial V / \partial T)_{P,\left\{x_{i}\right\}}=-\rho^{-1}(\partial \rho / \partial T)_{P,\left\{x_{i}\right\}}$, Isobaric expansivity (coefficient of thermal expansion), as suggested by Rowlinson and Swinton [29]. The Green Book [296] suggests either $\alpha$, or $\alpha_{V}$, or $\gamma$

$\alpha_{\mathrm{S}}(T, \omega) \quad$ Empirical $\alpha$-function (Soave EOS), Eq. 170

$\alpha_{\mathrm{PR}}(T, \omega) \quad$ Empirical $\alpha$-function (Peng-Robinson EOS), Eq. 176

$\beta_{T} \quad \equiv-V^{-1}(\partial V / \partial P)_{T,\left\{x_{i}\right\}}=\rho^{-1}(\partial \rho / \partial P)_{T,\left\{x_{i}\right\}}$, Isothermal compressibility, as suggested by Rowlinson and Swinton [29]. The Green Book [296] suggests $\kappa_{T}$ $\gamma_{V} \quad \equiv(\partial P / \partial T)_{V,\left\{x_{i}\right\}}=\alpha_{P} / \beta_{T}$, Isochoric thermal pressure coefficient, as suggested by Rowlinson and Swinton [29]. The Green Book [296] suggests $\beta$

$\gamma_{\sigma} \quad \equiv(\partial P / \partial T)_{\sigma}$, Slope of the vapor pressure curve, $\mathrm{d} P_{\sigma} / \mathrm{d} T$, as suggested by Rowlinson and Swinton [29]; not mentioned in the Green Book [296]

$\gamma_{i}^{\mathrm{LR}} \quad=\gamma_{i}^{\mathrm{LR}}\left(T, P, x_{i}\right)$, Activity coefficient of component $i$ of a binary mixture/solution based on the Lewis-Randall (LR) convention. The Green Book [296] suggests $f_{i}$ (same symbol as for fugacity!) 
$\gamma_{i}^{\mathrm{HL}} \quad=\gamma_{i}^{\mathrm{HL}}\left(T, P, x_{i}\right)$, Activity coefficient of component $i$ of a binary mixture/solution based on the Henry's law (HL) convention. The Green Book [296] suggests $\gamma_{x, i}$

$\Gamma \quad \equiv S-\frac{P}{T} V+\sum_{i}^{c} \frac{\mu_{i}}{T} x_{i}=\frac{1}{T} U$, molar unnamed primary function, see Table 3; not mentioned in the Green Book [296]

$\Gamma \quad$ Parameter used in Eqs. 286-288

$\delta \quad$ Parameter in a generalized vdW EOS, Eq. 161

$\delta \quad \equiv \rho_{n} / \rho_{n, \mathrm{c}}=V_{\mathrm{c}} / V$, Reduced amount density, i.e. inverse reduced molar volume, used in the fundamental equation Eq. 187

$\delta_{i j} \quad \equiv 2 B_{i j}-\left(B_{i i}+B_{j j}\right)$, Eq. 248

$\varepsilon \quad$ Parameter in a general vdW EOS, Eq. 161

$\eta \quad$ Parameter in a general vdW EOS, Eq. 161

$\theta \quad$ Parameter in a general vdW EOS, Eq. 161

$\Theta \quad \equiv S-\frac{P}{T} V=\frac{1}{T} U-\sum_{i}^{c} \frac{\mu_{i}}{T} x_{i}$, Molar unnamed primary function, see Table 3; not mentioned in the Green Book [296]

$\lambda_{i j}-\lambda_{i i} \quad$ Characteristic interaction energy difference, related to the Wilson parameter $\Lambda_{i j}$ via Eq. 290

$\Lambda$ $\equiv S+\sum_{i}^{c} \frac{\mu_{i}}{T} x_{i}=\frac{1}{T} U+\frac{P}{T} V$, Molar unnamed primary function, see Table 3; not mentioned in the Green Book [296]

$\Lambda_{12}, \Lambda_{21} \quad$ Adjustable Wilson parameters, Eqs. 285 and 290

$\mu_{i} \quad$ Chemical potential of component $i$ of a mixture/solution, Eqs. 11, 52a and $52 \mathrm{~b}$

$\mu_{i}^{\mathrm{E}} \quad=G_{i}^{\mathrm{E}}=R T \ln \gamma_{i}^{\mathrm{LR}}$, Excess chemical potential of component $i$ of a mixture/ solution, LR convention, Eq. 237

$\rho \quad \equiv m /(n V)=m_{\mathrm{m}} / V$, Mass density

$\rho_{n} \quad \equiv n /(n V)=1 / V=\rho / m_{\mathrm{m}}$, Amount(-of-substance) density

$\rho_{N} \quad \equiv N /(n V)=N_{A} / V=N_{A} \rho_{n}=N_{A} \rho / m_{\mathrm{m}}$, Number density. The Green Book [296] suggests $C$ or $n$

$\tau \quad \equiv T_{\mathrm{c}} / T=1 / T_{\mathrm{r}}$, Dimensionless parameter, i.e. inverse reduced temperature, used in the fundamental equation Eq. 187

$\tau \quad \equiv T_{\text {ref }} / T$, Dimensionless parameter, Eqs. 268-271

$\phi^{\pi} \quad$ Overall fugacity coefficient of a solution in phase $\pi$, Eqs. 135 and 136

$\phi_{i}^{\mathrm{L}}, \phi_{i}^{\mathrm{V}} \quad$ Fugacity coefficient of component $i$ in solution, liquid (L) phase or vapor (V) phase, Eqs. 129 and 130

$\phi_{i}^{\mathrm{L}, *}, \phi_{i}^{\mathrm{V}, *} \quad$ Fugacity coefficient of pure substance $i$ in the liquid $(\mathrm{L})$ phase or the vapor (V) phase, Eqs. 132 and 133

$\phi_{i}^{\mathrm{L}, \infty}(T, P) \quad$ Fugacity coefficient of component $i$ at infinite dilution in a binary liquid (L) phase, Eq. 310

$\phi_{2}^{\mathrm{V}, \infty}(T, P) \quad$ Fugacity coefficient of component 2 at infinite dilution in a binary vapor (V) phase, Eq. $153 \mathrm{c}$

$\Phi \quad \equiv S-\frac{1}{T} U-\frac{P}{T} V=-\sum_{i}^{c} \frac{\mu_{i}}{T} x_{i}$, Molar Planck function, Table 3. The Green Book [296] suggests $Y^{i}$

$\Phi_{i} \quad$ Correction term in the VLE relation, Eq. 246

$\Psi \quad \equiv S-\frac{1}{T} U=\frac{P}{T} V-\sum_{i}^{c} \frac{\mu_{i}}{T} x_{i}$, Molar Massieu function, see Table 3 . The Green Book [296] suggests $J$ 


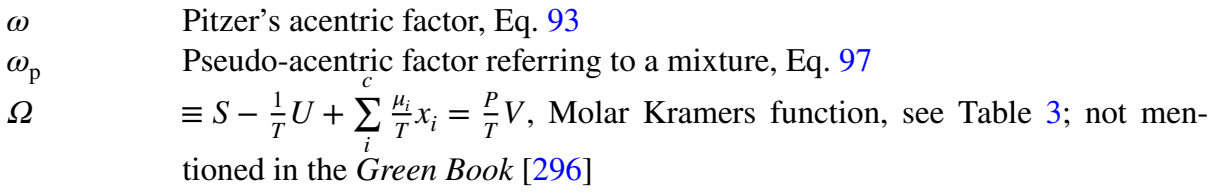

\section{Subscripts}

app Indicates an apparent molar property

c Indicates a critical property

$i, j, k$ General indices; usually $i$ or $j$ denote components in a mixture/solution; double indices $i i$ and $j j$ frequently indicate pure-substance properties, or characterize interaction between like molecules; in contradistinction, $i j$ frequently indicates a mixture quantity, or characterizes interaction between unlike molecules (cross properties) in a mixture/solution

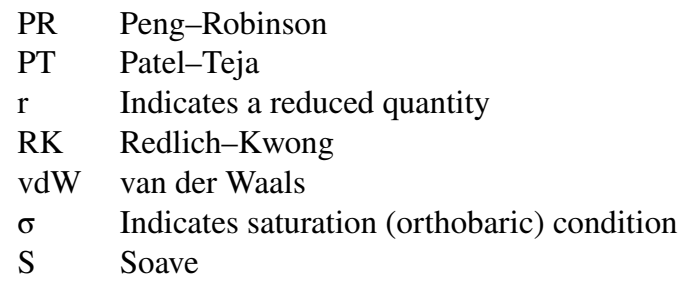

\section{Superscripts}

\section{E Excess property}

HL Indicates ideal-solution behavior based on Henry's law, Eq. 296

id Ideal solution, see Eqs. 196 and 197, or, alternatively, see Eq. 296

L Liquid phase

LR Indicates ideal-solution behavior based on the Lewis-Randall rule, Eq. 196

pg Indicates perfect-gas state (ideal-gas state)

$\mathrm{r} \quad$ Indicates a residual property in $\left(T, V,\left\{x_{i}\right\}\right)$-space

$\mathrm{R} \quad$ Indicates a residual property in $\left(T, P,\left\{x_{i}\right\}\right)$-space

t Indicates a total property $M^{\mathrm{t}}=n M$

V Vapor phase

* Indicates a pure-substance property

$\infty$ Indicates infinite dilution

$\pi \quad$ Indicates a phase, say, liquid (L) or vapor (V)

Funding Open access funding provided by University of Vienna.

Open Access This article is licensed under a Creative Commons Attribution 4.0 International License, which permits use, sharing, adaptation, distribution and reproduction in any medium or format, as long as you give appropriate credit to the original author(s) and the source, provide a link to the Creative Commons 
licence, and indicate if changes were made. The images or other third party material in this article are included in the article's Creative Commons licence, unless indicated otherwise in a credit line to the material. If material is not included in the article's Creative Commons licence and your intended use is not permitted by statutory regulation or exceeds the permitted use, you will need to obtain permission directly from the copyright holder. To view a copy of this licence, visit http://creativecommons.org/licenses/by/4.0/.

\section{References}

1. Prausnitz, J.M., Lichtenthaler, R.N., de Azevedo, E.G.: Molecular Thermodynamics of Fluid Phase Equilibria, 3rd edn. Prentice Hall PTR, New York (1999)

2. Kister, H.Z.: Distillation Operation. McGraw-Hill, New York (1990)

3. Kister, H.Z.: Distillation Design. McGraw-Hill, New York (1992)

4. Kister, H.Z.: Distillation Troubleshooting. McGraw-Hill, New York (2006)

5. McCabe, W.L., Smith, J.C., Harriott, P.: Unit Operations of Chemical Engineering, 7th edn. McGrawHill, New York (2006)

6. Letcher, T.M. (ed.): Developments and Applications in Solubility. The Royal Society of Chemistry/ IUPAC, Cambridge (2007)

7. Seader, J.D., Henley, E.J., Roper, D.K.: Separation Process Principles with Applications Using Process Simulators, 4th edn. Wiley, New York (2016)

8. Cantor, C.R., Schimmel, P.R.: Biophysical Chemistry: Part I: The Conformation of Biological Macromolecules. W.H. Freeman and Company, San Francisco (1980)

9. Cantor, C.R., Schimmel, P.R.: Biophysical Chemistry: Part II: Techniques for the Study of Biological Structure and Function. W.H. Freeman and Company, San Francisco (1980)

10. Cantor, C.R., Schimmel, P.R.: Biophysical Chemistry: Part III: The Behavior of Biological Macromolecules. W.H. Freeman and Company, San Francisco (1980)

11. Barrat, J.-L., Hansen, J.-P.: Basic Concepts for Simple and Complex Liquids. Cambridge University Press, Cambridge (2003)

12. Ben-Naim, A.: Molecular Theory of Solutions. Oxford University Press, Oxford (2006)

13. Ben-Naim, A.: Molecular Theory of Water and Aqueous Solutions: Part I: Understanding Water. World Scientific Publishing, Singapore (2009)

14. Ben-Naim, A.: Molecular Theory of Water and Aqueous Solutions: Part II: The Role of Water in Protein Folding. Self-Assembly and Molecular Recognition. World Scientific Publishing, Singapore (2011)

15. Marcus, Y.: Ions in Water and Biophysical Implications: From Chaos to Cosmos. Springer, Dordrecht (2012)

16. Sandler, S.I.: Chemical, Biochemical, and Engineering Thermodynamics, 5th edn. Wiley, New York (2017)

17. Van Ness, H.C., Abbott, M.M.: Classical Thermodynamics of Nonelectrolyte Solutions: With Applications to Phase Equilibria. McGraw-Hill Book Company, New York (1982)

18. Smith, J.M., Van Ness, H.C., Abbott, M.M., Swihart, M.T.: Introduction to Chemical Engineering Thermodynamics, 8th edn. McGraw-Hill Education, New York (2018)

19. Brochard-Wyart, F., Nassoy, P., Puech, P.-H.: Essentials of Soft Matter Science. CRC Press, Boca Raton (2020)

20. Hála, E., Pick, J., Fried, V., Vilím, O.: Vapour-Liquid Equilibrium, 2nd edn. Pergamon Press, New York (1968)

21. Marcus, Y.: Supercritical Water: A Green Solvent: Properties and Uses. Wiley, Hoboken (2012)

22. Manahan, S.E.: Environmental Chemistry, 10th edn. CRC Press, Boca Raton (2017)

23. Popper, K.R.: The Logic of Scientific Discovery. Routledge, London (2002)

24. van der Waals, J.D.: Over de continuiteit van den gas- en vloeistoftoestand. Doctoral thesis in wis- en natuurkunde. University of Leiden, The Netherlands (1873)

25. Kipnis, A.Y., Yavelov, B.E., Rowlinson, J.S.: Van der Waals and Molecular Science. Clarendon Press, Oxford (1996)

26. Rowlinson, J.S.: Cohesion. A Scientific History of Intermolecular Forces. Cambridge University Press, Cambridge (2002)

27. van der Waals, J.D.: Molekulartheorie eines Körpers, der aus zwei verschiedenen Stoffen besteht. Z. Physik. Chem. 5, 133-173 (1890)

28. Rowlinson, J.S.: Legacy of van der Waals. Nature 244, 414-417 (1973) 
29. Rowlinson, J.S., Swinton, F.L.: Liquids and Liquid Mixtures, 3rd edn. Butterworth Scientific, London (1982)

30. Callen, H.B.: Thermodynamics and an Introduction to Thermostatistics, 2nd edn. Wiley, New York (1985)

31. Domb, C.: The Critical Point: A Historical Introduction to the Modern Theory of Critical Phenomena. CRC Press, Boca Raton (2017)

32. Moldover, M.R., Sengers, J.V., Gammon, R.W., Hocken, R.J.: Gravity effects in fluids near the gasliquid critical point. Rev. Mod. Phys. 51, 79-99 (1979)

33. Jany, P.: Thermophysical property measurements near the critical point. Exp. Therm. Fluid Sci. 3, 124-137 (1990)

34. Carlès, P.: A brief review of the thermophysical properties of supercritical fluids. J. Supercrit. Fluids 53, 2-11 (2010)

35. Barmatz, M., Hahn, I., Lipa, J.A., Duncan, R.V.: Critical phenomena in microgravity: past, present, and future. Rev. Mod. Phys. 79, 1-52 (2007)

36. Pitzer, K.S.: Corresponding states for perfect liquids. J. Chem. Phys. 7, 583-590 (1939)

37. Wilhelm, E.: Internal energy and enthalpy: introduction, concepts and selected applications. In: Wilhelm, E., Letcher, T.M. (eds.) Enthalpy and Internal Energy: Liquids, Solutions and Vapours, pp. 1-61. The Royal Society of Chemistry/IACT, Cambridge (2018)

38. Alberty, R.A.: Legendre transforms in chemical thermodynamics. Chem. Rev. 94, 1457-1482 (1994)

39. Alberty, R.A., Barthel, J.M.G., Cohen, E.R., Ewing, M.B., Goldberg, R.N., Wilhelm, E.: Use of Legendre transforms in chemical thermodynamics (IUPAC Technical Report, IUPAC Committee on Legendre Transforms in Chemical Thermodynamics). Pure Appl. Chem. 73, 1349-1380 (2001)

40. Zia, R.K.P., Redish, E.F., McKay, S.R.: Making sense of the Legendre transform. Am. J. Phys. 77, 614-622 (2009)

41. Maitland, G.C., Rigby, M., Smith, E.B., Wakeham, W.A.: Intermolecular Forces: Their Origin and Determination. Clarendon Press, Oxford (1981)

42. Gray, C.G., Gubbins, K.E.: Theory of Molecular Fluids: Volume 1: Fundamentals. Clarendon Press, Oxford (1984)

43. Gray, C.G., Gubbins, K.E., Joslin, C.G.: Theory of Molecular Fluids: Volume 2: Applications. Oxford University Press, Oxford (2011)

44. Mohr, J.P., Newell, D.B., Taylor, B.N., Tiesinga, E.: Data and analysis for the CODATA 2017 special fundamental constants adjustment. Metrologia 55, 125-146 (2018)

45. Newell, D.B., Cabiati, F., Fischer, J., Fujii, K., Karshenboim, S.G., Margolis, H.S., de Mirandés, E., Mohr, J.P., Nez, F., Pachucki, K., Quinn, T.J., Taylor, B.N., Wang, M., Wood, B.M., Zhang, Z.: The CODATA 2017 values of $h, e, k$, and $N_{\mathrm{A}}$ for the revision of the SI. Metrologia 55, L13-L16 (2018)

46. Wilhelm, E.: Mitigating complexity: cohesion parameters and related topics. I: the Hildebrand solubility parameter. J. Solution Chem. 47, 1626-1709 (2018)

47. Guggenheim, E.A.: The principle of corresponding states. J. Chem. Phys. 13, 253-261 (1945)

48. Sterbacek, A., Biskup, B., Tausk, P.: Calculation of Properties Using Corresponding States Methods. Elsevier, Amsterdam (1979)

49. Poling, B.E., Prausnitz, J.M., O’Connell, J.P.: The Properties of Gases and Liquids, 5th edn. McGrawHill, New York (2001)

50. Ely, J.F.: The corresponding-states principle. In: Goodwin, A.R.H., Sengers, J.V., Peters, C.J. (eds.) Applied Thermodynamics of Fluids, pp. 135-171. The Royal Society of Chemistry/IUPAC \& IACT, Cambridge (2010)

51. Pitzer, K.S.: The volumetric and thermodynamic properties of fluids: I: theoretical basis and virial coefficients. J. Am. Chem. Soc. 77, 3427-3433 (1955)

52. Pitzer, K.S., Lippmann, D.Z., Curl, R.F., Jr., Huggins, C.M., Peterson, D.E.: The volumetric and thermodynamic properties of fluids: II: compressibility factor, vapor pressure and entropy of vaporization. J. Am. Chem. Soc. 77, 3433-3440 (1955)

53. Pitzer, K.S., Curl, R.F., Jr.: The volumetric and thermodynamic properties of fluids: III: empirical equation for the second virial coefficient. J. Am. Chem. Soc. 79, 2369-2370 (1957)

54. Curl, R.F., Jr., Pitzer, K.S.: Volumetric and thermodynamic properties of fluids: enthalpy, free energy, and entropy. Ind. Eng. Chem. 50, 265-274 (1958)

55. Pitzer, K.S.: Origin of the acentric factor. In: Storvick, T.S., Sandler, S.I. (eds.) Phase Equilibria and Fluid Properties in the Chemical Industry: Estimation and Correlation, ACS Symposium Series 60, pp. 1-10. American Chemical Society, Washington, DC (1977)

56. Lee, B.I., Kesler, M.G.: A generalized thermodynamic correlation based on three-parameter corresponding states. AIChE J. 21, 510-527 (1975)

57. Lee, B.I., Kesler, M.G.: Erratum. AIChE J. 21, 1040 (1975) 
58. Lee, B.I., Kesler, M.G.: Erratum. AIChE J. 21, 1237 (1975)

59. Kay, W.B.: Densities of hydrocarbon gases and vapors. Ind. Eng. Chem. 28, 1014-1019 (1936)

60. Kontogeorgis, G.M., Folas, G.K.: Thermodynamic Models for Industrial Applications: From Classical and Advanced Mixing Rules to Association Theories. Wiley, Chichester (2010)

61. Economou, I.E.: Cubic and generalized van der Waals equations of state. In: Goodwin, A.R.H., Sengers, J.V., Peters, C.J. (eds.) Applied Thermodynamics of Fluids, pp. 53-83. The Royal Society of Chemistry/IUPAC \& IACT, Cambridge (2010)

62. McCabe, C., Galindo, A.: SAFT associating fluids and fluid mixtures. In: Goodwin, A.R.H., Sengers, J.V., Peters, C.J. (eds.) Applied Thermodynamics of Fluids, pp. 215-279. The Royal Society of Chemistry/IUPAC \& IACT, Cambridge (2010)

63. Lemmon, E.W., Span, R.: Multi-parameter equations of state for pure fluids and mixtures. In: Goodwin, A.R.H., Sengers, J.V., Peters, C.J. (eds.) Applied Thermodynamics of Fluids, pp. 394-432. The Royal Society of Chemistry/IUPAC \& IACT, Cambridge (2010)

64. Span, R., Lemmon, E.W.: Volumetric properties from multiparameter equations of state. In: Wilhelm, E., Letcher, T.M. (eds.) Volume Properties: Liquids, Solutions and Vapours, pp. 135-151. The Royal Society of Chemistry/IUPAC \& IACT, Cambridge (2015)

65. Novák, J.P., Růžička, K., Fulem, M.: Calculation of thermodynamic functions from volumetric properties. In: Wilhelm, E., Letcher, T.M. (eds.) Volume Properties: Liquids, Solutions and Vapours, pp. 476-492. The Royal Society of Chemistry/IUPAC \& IACT, Cambridge (2015)

66. Wilhelm, E.: Solubilities, fugacities and all that in solution chemistry. J. Solution Chem. 44, 10041061 (2015)

67. Lewis, G.N.: The law of physico-chemical change. Proc. Am. Acad. Arts Sci. 37, 49-69 (1901)

68. Wilhelm, E.: What you always wanted to know about heat capacities, but were afraid to ask. J. Solution Chem. 39, 1777-1818 (2010)

69. Frenkel, M., Marsh, K.N., Kabo, K.J., Wilhoit, A.C., Roganov, G.N.: Thermodynamics of Organic Compounds in the Gas State, Vols. I and II. Thermodynamics Research Center, The Texas A\&M System, College Station (1994)

70. Dortmund Data Bank Software and Separation Technology. http://www.ddbst.de

71. Schäfer, K., Lax, E. (eds.): Kalorische Zustandsgrößen. Landolt-Börnstein, 6. Auflage, II. Band, 4. Teil. Springer, Berlin (1961)

72. Shimanouchi, T.: Tables of molecular vibrational frequencies: consolidated volume I. Nat. Stand. Ref. Data Ser., Nat. Bur. Stand. 39, 48 (1972)

73. Shimanouchi, T.: Tables of molecular vibrational frequencies: consolidated volume II. J. Phys. Chem. Ref. Data 6, 993-1102 (1977)

74. Shimanouchi, T., Matsuura, H., Ogawa, Y., Harada, I.: Tables of molecular vibrational frequencies: part 10. J. Phys. Chem. Ref. Data 9, 1149-1254 (1980)

75. Benson, S.W., Cruickshank, F.R., Golden, D.M., Haugen, G.R., O’Neal, H.E., Rodgers, A.S., Shaw, R., Walsh, R.: Additivity rules for the estimation of thermochemical properties. Chem. Rev. 69, 279324 (1969)

76. Joback, K.G., Reid, R.C.: Estimation of pure-component properties from group-contributions. Chem. Eng. Commun. 57, 223-243 (1987)

77. Constantinou, L., Gani, R.: New group contribution method for estimating properties of pure compounds. AIChE J. 40, 1697-1710 (1994)

78. Valderrama, J.O.: The state of the cubic equation of state. Ind. Eng. Chem. Res. 42, 1603-1618 (2003)

79. Redlich, O., Kwong, J.N.S.: On the thermodynamics of solutions: V: an equation of state. Fugacities of gaseous solutions. Chem. Rev. 44, 233-244 (1949)

80. Peng, D.Y., Robinson, D.B.: A new two-constant equation of state. Ind. Eng. Chem. Fundam. 15, 59-64 (1976)

81. Tsonopoulos, C., Heidman, J.L.: From Redlich-Kwong to the present. Fluid Phase Equilib. 24, 1-23 (1985)

82. Soave, G.: Equilibrium constants from a modified Redlich-Kwong equation of state. Chem. Eng. Sci. 27, 1197-1203 (1972)

83. Soave, G.: Improvement of the van der Waals equation of state. Chem. Eng. Sci. 39, 343-355 (1984)

84. Graboski, M.S., Daubert, T.E.: A modified Soave equation of state for phase equilibrium calculations: 1: hydrocarbon systems. Ind. Eng. Chem. Process Des. Dev. 17, 443-448 (1978)

85. Graboski, M.S., Daubert, T.E.: A modified Soave equation of state for phase equilibrium calculations: 2: systems containing $\mathrm{CO}_{2}, \mathrm{H}_{2} \mathrm{~S}, \mathrm{~N}_{2}$, and CO. Ind. Eng. Chem. Process Des. Dev. 17, $448-454$ (1978)

86. Graboski, M.S., Daubert, T.E.: A modified Soave equation of state for phase equilibrium calculations. 3: systems containing hydrogen. Ind. Eng. Chem. Process Des. Dev. 18, 300-306 (1979) 
87. Soave, G.: Improving the treatment of heavy hydrocarbons by the SRK EOS. Fluid Phase Equilib. 84, 339-342 (1983)

88. Privat, R., Visvonte, M., Zazoua-Khames, A., Jaubert, J.-N., Gani, R.: Analysis and prediction of the alpha-function parameters used in cubic equations of state. Chem. Eng. Sci. 126, 584-603 (2015)

89. Le Guennec, Y., Lasala, S., Privat, R., Jaubert, J.-N.: A consistency test for $\alpha$-functions of cubic equations of state. Fluid Phase Equilib. 427, 513-538 (2016)

90. Le Guennec, Y., Privat, R., Lasala, S., Jaubert, J.-N.: On the imperative need to use a consistent $\alpha$-function for the prediction pure-compound supercritical properties with a cubic equation of state. Fluid Phase Equilib. 445, 45-53 (2017)

91. Wilhelm, E.: Recent advances in molecular thermodynamics of fluid mixtures. Progr. Chem. Eng. A, Fundam. Chem. Eng. 21, 45-93 (1983)

92. Patel, N.C., Teja, A.S.: A new cubic equation of state for fluids and fluid mixtures. Chem. Eng. Sci. 37, 463-473 (1982)

93. Kohler, F., Fischer, J., Wilhelm, E.: Intermolecular force parameters for unlike pairs. J. Molec. Struct. 84, 245-250 (1982)

94. Goodwin, A.R.H., Sandler, S.I.: Mixing and combining rules. In: Goodwin, A.R.H., Sengers, J.V., Peters, C.J. (eds.) Applied Thermodynamics of Fluids, pp. 84-134. The Royal Society of Chemistry/ IUPAC \& IACT, Cambridge (2010)

95. Galitzine, B.: Über das Dalton'sche Gesetz: III. Theil: Theoretische Untersuchungen. Ann. Physik u. Chem., Neue Folge 41, 770-800 (1890)

96. Berthelot, D.: Sur le mélange des gaz. C. R. Seances Acad. Sci. 126, 1703-1706 (1898)

97. Berthelot, D.: Sur le mélange des gaz. C. R. Seances Acad. Sci. 126, 1857-1858 (1898)

98. Trusler, J.P.M.: The virial equation of state. In: Goodwin, A.R.H., Sengers, J.V., Peters, C.J. (eds.) Applied Thermodynamics of Fluids, pp. 33-52. The Royal Society of Chemistry/IUPAC \& IACT, Cambridge (2010)

99. Benedict, M., Webb, G.B., Rubin, L.C.: An empirical equation for thermodynamic properties of light hydrocarbons and their mixtures: I: methane, ethane, propane and butane. J. Chem. Phys. 8, 334-345 (1940)

100. Benedict, M., Webb, G.B., Rubin, L.C.: An empirical equation for thermodynamic properties of light hydrocarbons and their mixtures: II: mixtures of methane, ethane, propane and butane. J. Chem. Phys. 10, 747-758 (1942)

101. Benedict, M., Webb, G.B., Rubin, L.C.: An empirical equation for thermodynamic properties of light hydrocarbons and their mixtures: fugacities and liquid-vapor equilibria. Chem. Eng. Progress 47, 449-454 (1951)

102. Jacobson, R.T., Stewart, R.B.: Thermodynamic properties of nitrogen including liquid and vapor phases from $63 \mathrm{~K}$ to $2000 \mathrm{~K}$ with pressures to 10000 bar. J. Phys. Chem. Ref. Data 2, 757-922 (1973)

103. Bender, E.: Die Berechnung der Verdampfungsgleichgewichte von Mehrstoffsystemen bei hohen Drücken. Chemie-Ing.-Techn. 44, 576-582 (1972)

104. Bender, E.: An equation of state for predicting vapour-liquid equilibria of the system $\mathrm{N}_{2}-\mathrm{Ar}-\mathrm{O}_{2}$. Cryogenics 13, 11-18 (1973)

105. Bender, E.: Equations of state for ethylene and propylene. Cryogenics 15, 667-673 (1975)

106. Span, R., Lemmon, E.W., Jacobsen, R.T., Wagner, W., Yokozeki, A.: A reference equation of state for the thermodynamic properties of nitrogen for temperatures from 63.151 to $1000 \mathrm{~K}$ and pressures to 2200 MPa. J. Phys. Chem. Ref. Data 29, 1361-1433 (2000)

107. Span, R., Wagner, W., Lemmon, E.W., Jacobsen, R.T.: Multiparameter equations of state-recent trends and future challenges. Fluid Phase Equilib. 183-184, 1-20 (2001)

108. Wagner, W., Pruß, A.: The IAPWS formulation 1995 for the thermodynamic properties of the ordinary water substance for general and scientific use. J. Phys. Chem. Ref. Data 31, 387-535 (2002)

109. Lemmon, E.W., Tillner-Roth, R.: A Helmholtz energy equation of state for calculating the thermodynamic properties of fluid mixtures. Fluid Phase Equilib. 165, 1-21 (1999)

110. Lemmon, E.W., Jacobsen, R.T.: A generalized model for the thermodynamic properties of mixtures. Int. J. Thermophys. 20, 825-835 (1999)

111. Lemmon, E.W., Jacobsen, R.T.: Equations of state for mixtures of R-32, R-125, R-134a, R-143a, and R-152a. J. Phys. Chem. Ref. Data 33, 593-620 (2004)

112. Gernert, J., Jäger, A., Span, R.: Calculation of phase equilibria for multi-component mixtures using highly accurate Helmholtz energy equations of state. Fluid Phase Equilib. 375, 209-318 (2014)

113. Kunz, O., Wagner, W.: The GERG-2008 wide-range equation of state for natural gases and other mixtures: an expansion of GERG-2004. J. Chem. Eng. Data 57, 3032-3091 (2012) 
114. Deiters, U.K., Bell, I.H.: Unphysical critical curves of binary mixtures predicted with GERG models. Int. J. Thermophys. 41, 169/1-19 (2020)

115. Solubility Data Series (IUPAC): Volume 1: Clever, H.L.: Helium and Neon - Volume 53: Young, C.L.: Cummulative Index (for Vols. 40-52), published by Pergamon Press, Oxford; Volume 54: Acree, W.E., Jr.: Polycyclic Aromatic Hydrocarbons in Pure and Binary Solvents - Volume 65: Fritz, J.J., Königsberger, E.: Copper(I) Halides and Pseudohalides, published by Oxford University Press, Oxford

116. Solubility Data Series (IUPAC-NIST): Volume 66: Eysseltová, J., Dirkse, T.P.: Ammonium Phosphates. J. Phys. Chem. Ref. Data 27, 1289-1470 (1998) - and volumes above published in the Journal of Physical and Chemical Refence Data by the American Institute of Physics for the National Institute of Standards and Technology, Melville, NY

117. NIST SDR 4. NIST Thermophysical Properties of Hydrocarbon Mixtures Database: Version 3.2, NIST, Boulder, Colorado. http://www.nist.gov/srd/nist4.cfm

118. NIST SDR 10. NIST/ASME Steam Properties Database: Version 2.22, NIST, Boulder. http://www. nist.gov/srd/nist10.cfm

119. NIST SDR 103b: NIST ThermoData Engine Version 7.0 - Pure Compounds, Binary Mixtures, Ternary Mixtures, and Chemical Reactions, NIST, Boulder. http://www.nist.gov/srd/nist103b.cfm

120. NIST SDR 203. NIST Web Thermo Tables (WTT) - Professional Edition, NIST, Boulder. http:// www.nist.gov/srd/nistwebsub3.cfm

121. Wilhoit, R.C., Marsh, K.N., Hong, X. Gadalla N., Frenkel, M.: Densities of aliphatic hydrocarbons: alkanes. In: Marsh, K.N. (ed.) Landolt-Börnstein, Numerical Data and Functional Relationships in Science and Technology, New Series: Group IV: Physical Chemistry, Volume 8: Thermodynamic Properties of Organic Compounds and their Mixtures, Subvolume B. Springer, Berlin (1996)

122. Grolier, J.-P.E., Wormald, C.J., Fontaine, J.-C., Sosnkowska-Kehiaian, K., Kehiaian, H.V.: Heats of mixing and solution: binary liquid systems of nonelectrolytes. In: Kehiaian, H.V. (ed.) Landolt-Börnstein, Numerical Data and Functional Relationships in Science and Technology, New Series: Group IV: Physical Chemistry, Volume 10: Heats of Mixing, Subvolume A. Springer, Berlin (2004)

123. Wormald, C.J., Grolier, J.-P.E., Fontaine, J.-C., Sosnkowska-Kehiaian, K., Kehiaian, H.V.: Heats of mixing and solution: binary gaseous, liquid, near-critical, and supercritical fluid systems of nonelectrolytes. In: Kehiaian, H.V. (ed.) Landolt-Börnstein, Numerical Data and Functional Relationships in Science and Technology, New Series: Group IV: Physical Chemistry, Volume 10: Heats of Mixing and Solution, Subvolume B. Springer, Berlin (2005)

124. Cibulka, I., Fontaine, J.-C., Sosnkowska-Kehiaian, K., Kehiaian, H.V.: Binary liquid systems of nonelectrolytes I. Chapter 1. In: Kehiaian, H.V. (ed.) Landolt-Börnstein, Numerical Data and Functional Relationships in Science and Technology, New Series: Group IV: Physical Chemistry, Volume 26A: Binary Liquid Systems of Nonelectrolytes I. Springer, Berlin (2011)

125. Cibulka, I., Fontaine, J.-C., Sosnkowska-Kehiaian, K., Kehiaian, H.V.: Binary liquid systems of nonelectrolytes II. Introduction. In: Kehiaian, H.V. (ed.) Landolt-Börnstein, Numerical Data and Functional Relationships in Science and Technology, New Series: Group IV: Physical Chemistry, Volume 26B: Binary Liquid Systems of Nonelectrolytes II. Springer, Berlin (2012)

126. Cibulka, I., Fontaine, J.-C., Sosnkowska-Kehiaian, K., Kehiaian, H.V.: Binary liquid systems of nonelectrolytes III. Introduction. In: Kehiaian, H.V. (ed.) Landolt-Börnstein, Numerical Data and Functional Relationships in Science and Technology, New Series: Group IV: Physical Chemistry, Volume 26C: Binary Liquid Systems of Nonelectrolytes III. Springer, Berlin (2012)

127. Wichterle, I., Linek, J., Wagner, Z., Fontaine, J.-C., Sosnkowska-Kehiaian, K., Kehiaian, H.V.: Binary liquid systems of nonelectrolytes. Part 1. In: Kehiaian, H.V. (ed.) Landolt-Börnstein, Numerical Data and Functional Relationships in Science and Technology, New Series: Group IV: Physical Chemistry, Volume 13: Vapor-Liquid Equilibrium in Mixtures and Solutions, Subvolume A1. Springer, Berlin (2007)

128. Wichterle, I., Linek, J., Wagner, Z., Fontaine, J.-C., Sosnkowska-Kehiaian, K., Kehiaian, H.V.: Binary liquid systems of nonelectrolytes. Part 2. In: Kehiaian, H.V. (ed.) Landolt-Börnstein, Numerical Data and Functional Relationships in Science and Technology, New Series: Group IV: Physical Chemistry, Volume 13: Vapor-Liquid Equilibrium in Mixtures and Solutions, Subvolume A2. Springer, Berlin (2008)

129. Lacmann, R., Synowietz, C.: Nonaqueous Systems and Ternary Aqueous Systems. In: Schäfer, K1. (ed.) Landolt-Börnstein, Numerical Data and Functional Relationships in Science and Technology, New Series: Group IV: Macroscopic and Technical Properties of Matter, Volume 1: Densities of Liquid Systems, Part a. Springer, Berlin (1974)

130. Fredenslund, A., Rasmussen, P.: From UNIFAC to SUPERFAC - and back? Fluid Phase Equilib. 24, 115-150 (1985) 
131. Gmehling, J.: Present status and potential of group contribution methods for process development. J. Chem. Thermodyn. 41, 731-747 (2009)

132. Kang, J.W., Diky, V., Chirico, A.D., Magee, J.W., Muzny, C.D., Abdulagatov, L., Kazakov, A.F., Frenkel, M.: A new method for evaluation of UNIFAC interaction parameters. Fluid Phase Equilib. 309, 68-75 (2011)

133. Hector, D., Gmehling, J.: Present status of the modified UNIFAC model for the prediction of phase equilibria and excess enthalpies for systems with ionic liquids. Fluid Phase Equilib. 371, 82-92 (2014)

134. Kang, J.W., Diky, V., Frenkel, M.: New modified UNIFAC parameters using critically evaluated phase equilibrium data. Fluid Phase Equilib. 388, 128-141 (2015)

135. Constantinescu, D., Gmehling, J.: Further Development of modified UNIFAC (Dortmund): revision and extension. J. Chem. Eng. Data 61, 2738-2748 (2016)

136. Krooshof, G.J.P., Tuinier, R., de With, G.: On the calculation of nearest neighbors in activity coefficient models. Fluid Phase Equilib. 465, 10-23 (2018)

137. Kehiaian, H.V., Grolier, J.-P.E., Benson, G.C.: Thermodynamics of organic mixtures. A generalized quasichemical theory in terms of group surface interactions. J. Chim. Phys. 75, 1031-1048 (1978)

138. Kehiaian, H.V., Marongiu, B.: A comparative study of thermodynamic properties and molecular interactions in mono- and polychloroalkane $+\mathrm{n}$-alkane or + cyclohexane mixtures. Fluid Phase Equilib. 40, 23-78 (1988)

139. Marongiu, B., Piras, A., Porcedda, S., Tuveri, E.: Excess enthalpies of aromatic ether or aromatic ketone (1) $+n$-heptane (2) mixtures. J. Therm. Anal. Calorim. 92, 137-144 (2008)

140. Duce, C., Tiné, M.R., Lepori, L., Matteoli, E., Marongiu, B., Piras, A.: A comparative study of thermodynamic properties of binary mixtures containing perfluoroalkanes. J. Therm. Anal. Calorim. 92, $145-154$ (2008)

141. Bronneberg, R., Pfennig, A.: MOQUAC, a new expression for the excess Gibbs energy based on molecular orientations. Fluid Phase Equilib. 338, 63-77 (2013)

142. Bouillot, B., Teychené, S., Biscans, B.: An evaluation of thermodynamic models for the prediction of drug and drug-like molecule solubility in organic solvents. Fluid Phase Equilib. 309, 36-52 (2011)

143. Grensemann, H., Gmehling, J.: Performance of a conductor-like screening model for real solvents model in comparison to classical group contribution methods. Ind. Eng. Chem. Res. 44, 1610-1624 (2005)

144. Hsieh, C.-M., Sandler, S.I., Lin, S.-T.: Improvements of COSMO-SAC for vapor-liquid and liquidliquid equilibrium predictions. Fluid Phase Equilib. 297, 90-97 (2010)

145. Panayiotou, C.: Toward a COSMO equation-of-state model of fluids and their mixtures. Pure Appl. Chem. 83, 1221-1242 (2011)

146. Dong, Y., Huang, S., Guo, Y., Lei, Z.: COSMO-UNIFAC model for ionic liquids. AIChE J. 66, e16787/1-5 (2020)

147. Zhu, R., Taheri, M., Zhang, J., Lei, Z.: Extension of the COSMO-UNIFAC thermodynamic model. Ind. Eng. Chem. Res. 59, 1693-1701 (2020)

148. Prigogine, I., Defay, R., translated by Everett, D.H.: Chemical Thermodynamics. Longmans, Green and Co, London (1954)

149. Haase, R.: Thermodynamik der Mischphasen; mit einer Einführung in die Grundlagen der Thermodynamik. Springer, Berlin (1956)

150. Kortüm, G., Lachmann, H.: Einführung in die chemische Thermodynamik: Phänomenologische und statistische Behandlung, 7th edn. Verlag Chemie, Weinheim (1981)

151. Lewis, G.N., Randall, M.: Thermodynamics, 2nd edn.; revised by Pitzer. K.S., Brewer, L. McGrawHill, New York (1961)

152. Marsh, K.N., O'Hare, P.A.G. (eds.): Solution Calorimetry; Experimental Thermodynamics, Volume IV. Blackwell Scientific Publications/IUPAC, Oxford (1994)

153. Goodwin, A.R.H., Marsh, K.N. (eds.): Measurement of the Thermodynamic Properties of Single Phases; Experimental Thermodynamics, Volume VI. Elsevier/IUPAC, Amsterdam (2003)

154. Höhne, G., Hemminger, W., Flammersheim, H.-J.: Differential Scanning Calorimetry, 2nd edn. Springer, Berlin (2003)

155. Wilhelm, E., Letcher, T.M. (eds.): Heat Capacities: Liquids, Solutions and Vapours. The Royal Society of Chemistry/IUPAC \& IACT, Cambridge (2010)

156. Sarge, S.M., Höhne, G.W.H., Hemminger, W.: Calorimetry: Fundamentals, Instrumentation and Applications. Wiley-VCH, Weinheim (2014)

157. Wilhelm, E.: Chemical thermodynamics: a journey of many vistas. J. Solution Chem. 43, 525-576 (2014) 
158. Wilhelm, E., Letcher, T.M. (eds.): Volume Properties: Liquids, Solutions and Vapours. The Royal Society of Chemistry/IUPAC \& IACT, Cambridge (2015)

159. Wilhelm, E., Grolier, J.-P.E.: Excess volumes of liquid nonelectrolyte mixtures. In: Wilhelm, E., Letcher, T.M. (eds.) Volume Properties: Liquids, Solutions and Vapours, pp. 163-245. The Royal Society of Chemistry/IUPAC \& IACT, Cambridge (2015)

160. Wilhelm, E., Letcher, T.M. (eds.): Enthalpy and Internal Energy: Liquids, Solutions and Vapours. The Royal Society of Chemistry/IACT, London (2018)

161. Conder, J.R., Young, C.L.: Physicochemical Measurements by Gas Chromatography. Wiley, New York (1979)

162. Deiters, U.K., Schneider, G.M.: High pressure phase equilibria: experimental methods. Fluid Phase Equilib. 29, 145-160 (1986)

163. Fornari, R.E., Alessi, P., Kikic, I.: High pressure fluid phase equilibria: experimental methods and systems investigated (1978-1987). Fluid Phase Equilib. 57, 1-33 (1990)

164. Dohrn, R., Brunner, G.: High-pressure fluid-phase equilibria: experimental methods and systems investigated (1988-1993). Fluid Phase Equilib. 106, 213-282 (1995)

165. Kolb, B., Ettre, L.S.: Static Headspace-Gas Chromatography. Theory and Practice. Wiley, New York (1997)

166. Raal, J.D., Mühlbauer, A.L.: Phase Equilibria: Measurement and Computation. Taylor \& Francis, Washington, DC (1998)

167. Raal, J.D., Ramjugernath, D.: Vapour-liquid equilibrium at low pressure. In: Weir, A.D., de Loos, Th.W. (eds.) Measurement of the Thermodynamic Properties of Multiple Phases; Experimental Thermodynamics, Volume VII, pp. 71-87. Elsevier/IUPAC, Amsterdam (2005)

168. Richon, D., de Loos, Th.W.: Vapour-liquid equilibrium at high pressure. In: Weir, A.D., de Loos, Th.W. (eds.) Measurement of the Thermodynamic Properties of Multiple Phases; Experimental Thermodynamics, Volume VII, pp. 89-136. Elsevier/IUPAC, Amsterdam (2005)

169. Wilhelm, E.: Low-pressure solubility of gases in liquids. In: Weir, A.D., de Loos, Th.W. (eds.) Measurement of the Thermodynamic Properties of Multiple Phases; Experimental Thermodynamics, Volume VII, pp. 137-176. Elsevier/IUPAC, Amsterdam (2005)

170. Raal, J.D., Ramjugernath, D.: Measurement of limiting activity coefficients: non-analytical tools. In: Weir, A.D., de Loos, Th.W. (eds.) Measurement of the Thermodynamic Properties of Multiple Phases; Experimental Thermodynamics, Volume VII, pp. 339-357. Elsevier/IUPAC, Amsterdam (2005)

171. Dohnal, V.: Measurement of limiting activity coefficients using analytical tools. In: Weir, A.D., de Loos, Th.W. (eds.) Measurement of the Thermodynamic Properties of Multiple Phases; Experimental Thermodynamics, Volume VII, pp. 359-381. Elsevier/IUPAC, Amsterdam (2005)

172. Wilhelm, E., Letcher, T.M. (eds.): Gibbs Energy and Helmholtz Energy: Liquids, Solutions and Vapours. The Royal Society of Chemistry/IACT, London (2021)

173. Redlich, O., Kister, A.T.: Algebraic representation of properties and the classification of solutions. Ind. Eng. Chem. 40, 345-348 (1948)

174. Scatchard, G.: Equilibrium in non-electrolyte mixtures. Chem. Rev. 44, 7-35 (1949)

175. Wilson, G.M.: From Redlich-Kister to local composition models, group solutions, and electrolytes. Fluid Phase Equilib. 24, 77-85 (1985)

176. Myers, D.B., Scott, R.L.: Thermodynamic functions for nonelectrolyte solutions. Ind. Eng. Chem. 55, 43-46 (1963)

177. Marsh, K.N.: A general method for calculating the excess Gibbs free energy from isothermal vapourliquid equilibria. J. Chem. Thermodyn. 9, 719-724 (1977)

178. Baker, G.A., Graves-Morris, P.R.: Padé Approximants. Addison-Wesley, Reading (1981)

179. Klaus, R.L., Van Ness, H.C.: The orthogonal polynomial representation of thermodynamic excess functions. Chem. Eng. Prog. Symp. Ser. 63(81), 88-104 (1967)

180. Bale, C.W., Pelton, A.D.: Mathematical representation of thermodynamic properties in binary systems and solution of Gibbs-Duhem equation. Metall. Trans. 5, 2323-2337 (1974)

181. Pelton, A.D., Bale, C.W.: Legendre polynomial expansions of thermodynamic properties of binary solutions. Metall. Trans. A 17A, 1057-1063 (1986)

182. Kaptay, G.: A new equation for the temperature dependence of the excess Gibbs energy of solution phases. Calphad 28, 115-124 (2004)

183. Howald, R.A., Eliezer, I.: Comparison of the Bale-Pelton and Redlich-Kister representations of thermodynamic properties of binary systems. Metall. Trans. B 8B, 190-191 (1977)

184. Tomiska, J.: Zur Konversion der Anpassungen thermodynamischer Funktionen mittels einer Reihe Legendre'scher Polynome und der Potenzreihe. Calphad 5, 93-102 (1981) 
185. Kohler, F.: Zur Berechnung der thermodynamischen Daten eines ternären Systems aus den zugehörigen binären Systemen. Monatsh. Chem. 91, 738-740 (1960)

186. Hwa, S.C.P., Ziegler, W.T.: Temperature dependence of excess thermodynamic properties of ethanolmethylcyclohexane and ethanol-toluene systems. J. Phys. Chem. 70, 2572-2593 (1966)

187. Holzhauer, J.K., Ziegler, W.T.: Temperature dependence of excess thermodynamic properties of $n$-heptane-toluene, methylcyclohexane-toluene, and $n$-heptane-methylcyclohexane systems. J. Phys. Chem. 79, 590-604 (1975)

188. Wilhelm, E., Egger, W., Vencour, M., Roux, A.H., Polednicek, M., Grolier, J.-P.E.: Thermodynamics of liquid mixtures of a very polar and a non-polar aromatic: (benzonitrile + benzene, or toluene). J. Chem. Thermodyn. 30, 1509-1532 (1998)

189. Grolier, J.-P.E., Inglese, A., Wilhelm, E.: Excess molar heat capacities of (1,4-dioxane + an $n$-alkane): an unusual composition dependence. J. Chem. Thermodyn. 16, 67-71 (1984); the experimental results, that is, W-shaped curves at $298.15 \mathrm{~K}$ and atmospheric pressure, were already communicated at the 37th Calorimetry Conference in Snowbird, Utah, 20 to 23 July, 1982, Paper No. 54

190. Sandler, S.I.: Infinite dilution activity coefficients in environmental and biochemical engineering. Fluid Phase Equilib. 116, 343-353 (1996)

191. Turner, L.H., Chiew, Y.C., Ahlert, R.C., Kosson, D.S.: Measuring vapor-liquid equilibrium for aqueous-organic systems: review and a new technique. AIChE J. 42, 1772-1788 (1996)

192. Kojima, K., Zhang, S., Hiaki, T.: Measuring methods of infinite dilution activity coefficients and a database for systems including water. Fluid Phase Equilib. 131, 145-179 (1997)

193. Schreiber, L.B., Eckert, C.A.: Use of infinite dilution activity coefficients with Wilson's equation. Ind. Eng. Chem. Process Des. Dev. 10, 572-576 (1971)

194. Letcher, T.M.: Activity coefficients at infinite dilution from gas-liquid chromatography. In: McGlashan, M.L. (ed.) Chemical Thermodynamics, Volume 2, A Specialist Periodical Report, pp. 46-70. The Chemical Society, London (1978)

195. Wisniak, J.: Max Margules - a cocktail of meteorology and thermodynamics. J. Phase Equilib. 24, 103-109 (2003)

196. van Klooster, H.S.: J. J. van Laar, pioneer in chemical thermodynamics. J. Chem. Educ. 39, 74-76 (1962)

197. Wisniak, J.: Johannes Jacobus van Laar: unappreciated scientist. Chem. Educ. 5, 335-339 (2000)

198. Wilson, G.M.: Vapor-liquid equilibrium XI: a new expression for the excess free energy of mixing. J. Am. Chem. Soc. 86, 127-130 (1964)

199. Renon, H., Prausnitz, J.M.: Local compositions in thermodynamic excess functions for liquid mixtures. AIChE J. 14, 135-144 (1968)

200. Renon, H., Prausnitz, J.M.: Estimation of parameters for the NRTL equation for excess Gibbs energies of strongly nonideal liquid mixtures. Ind. Eng. Chem. Process Des. Dev. 8, 413-419 (1969)

201. Abrams, D.S., Prausnitz, J.M.: Statistical thermodynamics of liquid mixtures: a new expression for the excess Gibbs energy of partly or completely miscible systems. AIChE J. 21, 116-128 (1975)

202. Maurer, G., Prausnitz, J.M.: On the derivation and extension of the UNIQUAC equation. Fluid Phase Equilib. 2, 91-99 (1978)

203. Anderson, T.F., Prausnitz, J.M.: Application of the UNIQUAC equation to calculation of multicomponent phase equilibria 1: vapor-liquid equilibria. Ind. Eng. Chem. Process Des. Dev. 17, 552-560 (1978)

204. Anderson, T.F., Prausnitz, J.M.: Application of the UNIQUAC equation to calculation of multicomponent phase equilibria 2: liquid-liquid equilibria. Ind. Eng. Chem. Process Des. Dev. 17, 561-567 (1978)

205. Kemény, S., Rasmussen, P.: A derivation of local composition expressions from partition functions. Fluid Phase Equilib. 7, 197-203 (1981)

206. Kehiaian, H.V.: Thermodynamik flüssiger Mischungen von Kohlenwasserstoffen mit verwandten Substanzen. Ber. Bunsenges. Physik. Chem. 81, 908-921 (1977)

207. Kehiaian, H.V.: Group contribution methods for liquid mixtures: A critical review. Fluid Phase Equilib. 13, 243-252 (1983)

208. Kehiaian, H.V.: Thermodynamics of binary liquid organic mixtures. Pure Appl. Chem. 57, 15-30 (1985)

209. Marongiu, B., Porcedda, S., Piras, A., Falconieri, D., Tiné, M.R.: The DISQUAC model. Rev. Roum. Chim. 56, 453-464 (2011)

210. Battino, R., Clever, H.L.: The solubility of gases in liquids. Chem. Rev. 66, 395-463 (1966)

211. Wilhelm, E., Battino, R.: Thermodynamic functions of the solubilities of gases in liquids at $25^{\circ} \mathrm{C}$. Chem. Rev. 73, 1-9 (1973) 
212. Kruis, A.: Gleichgewicht der Absorption von Gasen in Flüssigkeiten: Absorption in Flüssigkeiten von niedrigem Dampfdruck. In: Hausen, H. (ed.) Landolt-Börnstein, Zahlenwerte und Funktionen aus Physik, Chemie, Astronomie, Geophysik und Technik, 6. Auflage: IV. Band: Technik, 4. Teil: Wärmetechnik, Bandteil c1. Springer, Berlin (1976)

213. Kruis, A.: Gleichgewicht der Absorption von Gasen in Flüssigkeiten: Absorption in Flüssigkeiten von hohem Dampfdruck. In: Hausen, H. (ed.) Landolt-Börnstein, Zahlenwerte und Funktionen aus Physik, Chemie, Astronomie, Geophysik und Technik, 6. Aufllage: IV. Band: Technik, 4. Teil: Wärmetechnik, Bandteil c2. Springer, Berlin (1980)

214. Wilhelm, E., Battino, R., Wilcock, R.J.: Low-pressure solubility of gases in liquid water. Chem. Rev. 77, 219-262 (1977)

215. Mackay, D., Shiu, W.Y.: A critical review of Henry's law constants for chemicals of environmental interest. J. Phys. Chem. Ref. Data 10, 1175-1199 (1981)

216. Wilhelm, E.: Precision methods for the determination of the solubility of gases in liquids. CRC Crit. Rev. Analyt. Chem. 16, 129-175 (1985)

217. Wilhelm, E.: Dilute solutions of gases in liquids. Fluid Phase Equilib. 27, 233-261 (1986)

218. Wilhelm, E.: Thermodynamics of solutions, especially dilute solutions of nonelectrolytes. In: Teixeira-Dias, J.J.C. (ed.) Molecular Liquids: New Perspectives in Physics and Chemistry, pp. 175-206. Kluwer Academic Publishers, Dordrecht (1992)

219. Wilhelm, E.: The art and science of solubility measurements: what do we learn? Netsu Sokutai 39(2), 61-86 (2012)

220. Dymond, J.H., Marsh, K.N., Wilhoit, R.C., Wong, K.C.: Virial coefficients of pure gases. In: Frenkel, M., Marsh, K.N. (eds.) Landolt-Börnstein, Numerical Data and Functional Relationships in Science and Technology, New Series: Group IV: Physical Chemistry, Volume 21: Virial Coefficients of Pure Gases and Mixtures, Subvolume A. Springer, Heidelberg (2002)

221. Dymond, J.H., Marsh, K.N., Wilhoit, R.C.: Virial coefficients of mixtures. In: Frenkel, M., Marsh, K.N. (eds.) Landolt-Börnstein, Numerical Data and Functional Relationships in Science and Technology, New Series: Group IV: Physical Chemistry, Volume 21: Virial Coefficients of Pure Gases and Mixtures, Subvolume B. Springer, Heidelberg (2003)

222. Harvey, A.H., Lemmon, E.W.: Correlation for the second virial coefficient of water. J. Phys. Chem. Ref. Data 33, 369-376 (2004)

223. Tsonopoulos, C., Dymond, J.H.: Second virial coefficients of normal alkanes, linear 1-alkanols (and water), alkyl ethers, and their mixtures. Fluid Phase Equilib. 133, 11-34 (1997)

224. Moore, J.C., Battino, R., Rettich, T.R., Handa, Y.P., Wilhelm, E.: Partial molar volumes of "gases" at infinite dilution in water at 298.15 K. J. Chem. Eng. Data 27, 22-24 (1982)

225. Zhou, T., Battino, R.: Partial molar volumes of 13 gases in water at $298.15 \mathrm{~K}$ and 303.15 K. J. Chem. Eng. Data 46, 331-332 (2001)

226. Wilhelm, E., Battino, R.: Partial molar volumes of gases dissolved in liquids. In: Wilhelm, E., Letcher, T.M. (eds.) Volume Properties: Liquids, Solutions and Vapours, pp. 273-306. The Royal Society of Chemistry/IUPAC \& IACT, Cambridge (2015)

227. Cibulka, I., Heintz, A.: Partial molar volumes of air-component gases in binary liquid mixtures with n-alkanes and 1-alkanols at 298.15 K. Fluid Phase Equilib. 107, 235-255 (1995)

228. Izák, P., Cibulka, I., Heintz, A.: Partial molar volumes of air-component gases in several liquid n-alkanes and 1-alkanols at 31315 K. Fluid Phase Equilib. 109, 227-234 (1995)

229. Ashcroft, S.J., Ben Isa, M.: Effect of dissolved gases on the densities of hydrocarbons. J. Chem. Eng. Data 42, 1244-1248 (1997)

230. Albert, H.J., Wood, R.H.: High-precision flow densimeter for fluids at temperatures to $700 \mathrm{~K}$ and pressures to $40 \mathrm{MPa}$. Rev. Sci. Instrum. 55, 589-593 (1984)

231. Biggerstaff, D.R., Wood, R.H.: Apparent molar volumes of aqueous argon, ethylene, and xenon from 300 to 716 K. J. Phys. Chem. 92, 1988-1994 (1988)

232. Hnedkovský, L., Wood, R.H., Majer, V.: Volumes of aqueous solutions of $\mathrm{CH}_{4}, \mathrm{CO}_{2}, \mathrm{H}_{2} \mathrm{~S}$, and $\mathrm{NH}_{3}$ at temperatures from $298.15 \mathrm{~K}$ to $705 \mathrm{~K}$ and pressures to $35 \mathrm{MPa}$. J. Chem. Thermodyn. 28, 125-142 (1996)

233. Handa, Y.P., D’Arcy, P.J., Benson, G.C.: Partial molar volumes of gases dissolved in liquids: part II: a dilatometer for measuring infinite-dilution partial molar volumes, and results for 40 liquid-gas systems. Fluid Phase Equilib. 8, 181-196 (1982)

234. Bignell, N.: Partial molar volumes of atmospheric gases in water. J. Phys. Chem. 88, 5409-5412 (1984)

235. Bignell, N.: Precise density measurements of aqueous solutions of mixed nonpolar gases. J. Phys. Chem. 91, 1687-1690 (1987) 
236. Harvey, A.H., Kaplan, S.G., Burnett, J.H.: Effect of dissolved air on the density and refractive index of water. Int. J. Thermophys. 26, 1495-1514 (2005)

237. Wilhelm, E.: Caloric properties of dilute nonelectrolyte solutions. Thermochim. Acta 300, 159-168 (1997)

238. Wilhelm, E.: Thermodynamics of nonelectrolyte solubility. In: Letcher, T.M. (ed.) Developments and Applications in Solubility, pp. 3-18. The Royal Society of Chemistry/IUPAC, Cambridge (2007)

239. Wilhelm, E.: Aqueous solutions of nonelectrolytes: a molecular thermodynamics perspective. J. Therm. Anal. Calorim. 108, 547-558 (2012)

240. Wilhelm, E., Battino, R.: Partial molar heat capacity changes of gases in liquids. In: Wilhelm, E., Letcher, T.M. (eds.) Heat Capacities: Liquids, Solutions and Vapours, pp. 457-471. The Royal Society of Chemistry/IUPAC \& IACT, Cambridge (2010)

241. Wilhelm, E., Battino, R.: Enthalpy changes on solution of gases in liquids. In: Wilhelm, E., Letcher, T.M. (eds.) Enthalpy and Internal Energy: Liquids, Solutions and Vapours, pp. 269-298. The Royal Society of Chemistry/IACT, Cambridge (2018)

242. Clarke, E.C.W., Glew, D.N.: Evaluation of thermodynamic functions from equilibrium constants. Trans. Faraday Soc. 62, 539-547 (1966)

243. Bolton, P.D.: Calculation of thermodynamic functions from equilibrium data. J. Chem. Educ. 47, 638-641 (1970)

244. Wauchope, R.D., Haque, R.: Aqueous solutions of nonpolar compounds. Heat-capacity effects. Can. J. Chem. 50, 133-138 (1972)

245. Benson, B.B., Krause, D., Jr.: Empirical laws for dilute aqueous solutions of nonpolar gases. J. Chem. Phys. 64, 689-709 (1976)

246. Benson, B.B., Krause, D., Jr., Peterson, M.A.: The solubility and isotope fractionation of gases in dilute aqueous solutions I. Oxygen. J. Solution Chem. 8, 655-690 (1979)

247. Krause, D., Jr., Benson, B.B.: The solubility and isotopic fractionation of gases in dilute aqueous solutions. IIa: solubilities of the noble gases. J. Solution Chem. 18, 823-873 (1989)

248. Valentiner, S.: Über die Löslichkeit der Edelgase in Wasser. Z. Phys. 42, 253-264 (1927)

249. Crovetto, R., Fernández-Prini, R., Japas, M.L.: Solubilities of inert gases and methane in $\mathrm{H}_{2} \mathrm{O}$ and in $\mathrm{D}_{2} \mathrm{O}$ in the temperature range of 300 to $600 \mathrm{~K}$. J. Chem. Phys. 76, 1077-1086 (1982)

250. Harvey, A.H.: Semiempirical correlation for Henry's constants over large temperature ranges. AIChE J. 42, 1491-1494 (1996)

251. Fernández-Prini, R., Alvarez, J.L., Harvey, A.H.: Henry's constants and vapor-liquid distribution constants for gaseous solutes in $\mathrm{H}_{2} \mathrm{O}$ and in $\mathrm{D}_{2} \mathrm{O}$ at high temperatures. J. Phys. Chem. Ref. Data 32, 903-916 (2003)

252. Rettich, T.R., Handa, Y.P., Battino, R., Wilhelm, E.: Solubility of gases in liquids 13. High-precision determination of Henry's constants for methane and ethane in liquid water at 275 to $328 \mathrm{~K}$. J. Phys. Chem. 85, 3230-3237 (1981)

253. Tanford, C.: The Hydrophobic Effect: Formation of Micelles and Biological Membranes, 2nd edn. Wiley, New York (1980)

254. Ben-Naim, A.: Hydrophobic Interactions. Plenum Press, New York (1980)

255. Pratt, L.R.: Molecular theory of hydrophobic effects: "She is too mean to have her name repeated." Ann. Rev. Phys. Chem. 53, 409-436 (2002)

256. Southall, N.T., Dill, K.A., Haymet, A.D.J.: A view of the hydrophobic effect. J. Phys. Chem. B 106, 521-533 (2002)

257. Ben-Amotz, D., Underwood, R.: Unraveling water's entropic mysteries: a unified view of nonpolar, polar and ionic hydration. Acc. Chem. Res. 41, 957-967 (2008)

258. Ball, P.: Water as an active constituent in cell biology. Chem. Rev. 108, 74-108 (2008)

259. Paschek, D., Ludwig, R., Holzmann, J.: Computer simulation studies of heat capacity effects associated with hydrophobic effects. In: Wilhelm, E., Letcher, T.M. (eds.) Heat Capacities: Liquids, Solutions and Vapours, pp. 436-456. The Royal Society of Chemistry/IUPAC \& IACT, Cambridge (2010)

260. Hillyer, M.B., Gibb, B.C.: Molecular shape and the hydrophobic effect. Ann. Rev. Phys. Chem. 67, 307-329 (2016)

261. Ben-Amotz, D.: Water-mediated hydrophobic interactions. Ann. Rev. Phys. Chem. 67, 617-638 (2016)

262. Silverstein, T.P.: The hydrophobic effect: is water afraid or just not that interested? ChemTexts 6, 26/1-26 (2020)

263. Japas, M.L., Levelt Sengers, J.M.H.: Gas solubility and Henry's law near the solvent's critical point. AIChE J. 35, 705-713 (1989)

264. Benson, B.B., Krause, D., Jr.: A thermodynamic treatment of dilute solutions of gases in liquids. J. Solution Chem. 18, 803-821 (1989) 
265. Alexander, D.M.: A calorimetric measurement of the heats of solution of the inert gases in water. J. Phys. Chem. 63, 994-996 (1959)

266. Battino, R., Marsh, K.N.: An isothermal displacement calorimeter for the measurement of the enthalpy of solution of gases. Aust. J. Chem. 33, 1997-2003 (1980)

267. Gill, S.J., Wadsö, I.: Flow-microcalorimetric techniques for solution of slightly soluble gases. Enthalpy of solution of oxygen in water at 298.15 K. J. Chem. Thermodyn. 14, 905-919 (1982)

268. Dec, S.F., Gill, S.J.: Steady-state gas dissolution flow microcalorimeter for determination of heats of solution of slightly soluble gases in water. Rev. Sci. Instrum. 55, 765-772 (1984)

269. Olofsson, G., Oshodj, A.A., Qvarnström, E., Wadsö, I.: Calorimetric measurements on slightly soluble gases in water. Enthalpies of solution of helium, neon, argon, krypton, xenon, methane, ethane, propane, n-butane, and oxygen at 288.15, 298.15, and 308.15 K. J. Chem. Thermodyn. 16, 10411052 (1984)

270. Dec, S.F., Gill, S.J.: Heats of solution of gaseous hydrocarbons in water at $25^{\circ}$ C. J. Solution Chem. 13, 27-41 (1984)

271. Dec, S.F., Gill, S.J.: Enthalpies of aqueous solutions of noble gases at $25{ }^{\circ}$ C. J. Solution Chem. 14, 417-429 (1985)

272. Dec, S.F., Gill, S.J.: Heats of solution of gaseous hydrocarbons in water at 15,25 , and $35^{\circ}$ C. J. Solution Chem. 14, 827-836 (1985)

273. Naghibi, H., Dec, S.F., Gill, S.J.: Heat of solution of methane in water from 0 to $50{ }^{\circ}$ C. J. Phys. Chem. 90, 4621-4623 (1986)

274. Naghibi, H., Dec, S.F., Gill, S.J.: Heats of solution of ethane and propane in water from 0 to $50{ }^{\circ} \mathrm{C}$. J. Phys. Chem. 91, 245-248 (1987)

275. Naghibi, H., Ownby, D.W., Gill, S.J.: Enthalpies of solution of butanes in water from 5 to $45^{\circ}$ C. J. Chem. Eng. Data 32, 422-425 (1987)

276. Naghibi, H., Ownby, D.W., Gill, S.J.: Heats of solution of several freons in water from 5 to $45^{\circ} \mathrm{C}$. J. Solution Chem. 16, 171-179 (1987)

277. Hallén, D., Wadsö, I.: A new microcalorimetric vessel for dissolution of slightly soluble gases. Enthalpies of solution in water of carbon tetrafluoride and sulphur hexafluoride at 288.15, 298.15, and 308.15 K. J. Chem. Thermodyn. 21, 519-528 (1989)

278. Biggerstaff, D.R., White, D.E., Wood, R.H.: Heat capacities of aqueous argon from 306 to 578 K. J. Phys. Chem. 89, 4378-4381 (1985)

279. Biggerstaff, D.R., Wood, R.H.: Apparent molar heat capacities of aqueous argon, ethylene, and xenon at temperatures up to $720 \mathrm{~K}$ and pressures to $33 \mathrm{MPa}$. J. Phys. Chem. 92, 1994-2000 (1988)

280. Hnedkovský, L., Wood, R.H.: Apparent molar heat capacities of aqueous solutions of $\mathrm{CH}_{4}, \mathrm{CO}_{2}, \mathrm{H}_{2} \mathrm{~S}$, and $\mathrm{NH}_{3}$ at temperatures from $304 \mathrm{~K}$ to $704 \mathrm{~K}$ at a pressure of $28 \mathrm{MPa}$. J. Chem. Thermodyn. 29, 731-747 (1997)

281. Picker, P., Leduc, P.-A., Philip, P.R., Desnoyers, J.E.: Heat capacity of solutions by flow microcalorimetry. J. Chem. Thermodyn. 3, 631-642 (1971)

282. Barbero, J.A., McCurdy, K.G., Tremaine, P.R.: Apparent molal heat capacities and volumes of aqueous hydrogen sulfide and sodium hydrogen sulfide near $25{ }^{\circ} \mathrm{C}$ : the temperature dependence of $\mathrm{H}_{2} \mathrm{~S}$ ionization. Can. J. Chem. 60, 1872-1880 (1982)

283. Barbero, J.A., Hepler, L.G., McCurdy, K.G., Tremaine, P.R.: Thermodynamics of aqueous carbon dioxide and sulfur dioxide: heat capacities, volumes, and the temperature dependence of ionization. Can. J. Chem. 61, 2509-2519 (1983)

284. Battino, R.: Thermodynamics works! Enthalpy and heat capacity changes on solution from gas solubility data. J. Chem. Eng. Data 54, 301-304 (2009)

285. Rettich, T.R., Battino, R., Wilhelm, E.: Solubility of gases in liquids 15: high-precision determination of Henry coefficients for carbon monoxide in liquid water at 278 to $323 \mathrm{~K}$. Ber. Bunsenges. Phys. Chem. 86, 1128-1132 (1982)

286. Rettich, T.R., Battino, R., Wilhelm, E.: Solubility of gases in liquids 16: Henry's law coefficients for nitrogen in liquid water at 5 to $50^{\circ} \mathrm{C}$. J. Solution Chem. 13, 335-348 (1984)

287. Rettich, T.R., Battino, R., Wilhelm, E.: Solubility of gases in liquids 18: high-precision determination of Henry fugacities for argon in liquid water at 2 to $40^{\circ}$ C. J. Solution Chem. 21, 987-1004 (1992)

288. Rettich, T.R., Battino, R., Wilhelm, E.: Solubility of gases in liquids 22: high-precision determination of Henry's law constants of oxygen in liquid water from $\mathrm{T}=274 \mathrm{~K}$ to $\mathrm{T}=328 \mathrm{~K}$. J. Chem. Thermodyn. 32, 1145-1156 (2000)

289. Rettich, T.R., Battino, R., Wilhelm, E.: in preparation; communicated by E. W. at the 20th ICCT, Warsaw, Poland, August 3 - 8, 2008: p. 127, paper ES-RS-O-8

290. Ben-Naim, A.: Hydrophobic hydrophilic phenomena in biochemical processes. Biophys. Chem. 105, 183-193 (2003) 
291. Ben-Naim, A.: The rise and fall of the hydrophobic effect in protein folding and protein-protein association and molecular recognition. Open J. Biophys. 1, 1-7 (2011)

292. Wilhelm, E.: In appreciation of Professor Robert H. Wood. J. Solution Chem. 44, 908-911 (2015)

293. Wilhelm, E., Fischer, J.: In appreciation of Professor Friedrich Kohler. Fluid Phase Equilib. 48, VIIVIII (1989)

294. Grolier, J.-P.E., Wilhelm, E.: Obituary: Henry Vartan Kehiaian (1929-2009). Fluid Phase Equilib. 293, 261 (2010)

295. Grolier, J.-P.E., Wilhelm, E.: Obituary: Henry Vartan Kehiaian (1929-2009). J. Chem. Eng. Data 55, 1467 (2010)

296. International Union of Pure and Applied Chemistry: Quantities, Units and Symbols in Physical Chemistry, 3rd edn. RSC Publishing, Cambridge (2007)

297. Griffiths, R.B., Wheeler, J.C.: Critical points in multicomponent systems. Phys. Rev. A 2, 1047-1064 (1970)

298. Wilhelm, E.: Volumetric properties: introduction, concepts and selected applications. In: Wilhelm, E., Letcher, T.M. (eds.) Volume Properties: Liquids, Solutions and Vapours, pp. 1-72. The Royal Society of Chemistry/ IUPAC \& IACT, Cambridge (2015)

Publisher's Note Springer Nature remains neutral with regard to jurisdictional claims in published maps and institutional affiliations.

\section{Authors and Affiliations}

\section{Emmerich Wilhelm ${ }^{1}$}

Emmerich Wilhelm

emmerich.wilhelm@univie.ac.at

1 Institute of Materials Chemistry \& Research, University of Wien (Vienna), Währinger Straße 42, 1090 Wien (Vienna), Austria 